\title{
Single-cell multi-omics of human clonal hematopoiesis reveals that DNMT3A R882 mutations perturb early progenitor states through selective hypomethylation.
}

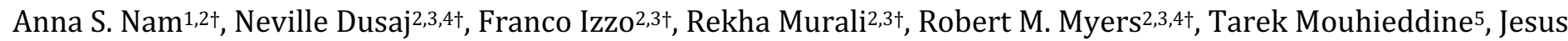
Sotelo 2,3, Salima Benbarche6, Michael Waarts6, Federico Gaiti2,3, Sabrin Tahri ${ }^{5}$, Ross Levine ${ }^{6}$, Omar Abdel-Wahab $^{6}$, Lucy A. Godley7, Ronan Chaligne ${ }^{2,3} \neq$, Irene Ghobrial $5^{*} \neq$, Dan A. Landau ${ }^{2,3,8^{*} \neq}$

1Department of Pathology and Laboratory Medicine, Weill Cornell Medicine; New York, NY, USA.

2New York Genome Center; New York, NY, USA.

3Division of Hematology and Medical Oncology, Department of Medicine and Meyer Cancer Center, Weill Cornell Medicine; New York, NY, USA. 4Tri-Institutional MD-PhD Program, Weill Cornell Medicine, Rockefeller University, Memorial Sloan Kettering Cancer Center; New York, NY, USA.

5Department of Medical Oncology, Dana-Farber Cancer Institute; Boston, MA, USA.

${ }^{6}$ Human Oncology and Pathogenesis Program, Memorial Sloan Kettering Cancer Center; New York, NY, USA.

7Section of Hematology/Oncology, Departments of Medicine and Human Genetics, The University of Chicago, Chicago, IL, USA.

8Institute for Computational Biomedicine, Weill Cornell Medicine; New York, NY, USA.

tContributed equally to this work; ${ }^{\ddagger}$ Jointly supervised this work; ${ }^{*}$ Corresponding author.

Correspondence: dlandau@nygenome.org and Irene_Ghobrial@dfci.harvard.edu

\section{ABSTRACT}

Somatic mutations in cancer genes have been ubiquitously detected in clonal expansions across healthy human tissue, including in clonal hematopoiesis. However, mutated and wildtype cells are morphologically and phenotypically similar, limiting the ability to link genotypes with cellular phenotypes. To overcome this limitation, we leveraged multi-modality single-cell sequencing, capturing the mutation with transcriptomes and methylomes in stem and progenitors from individuals with DNMT3A R882 mutated clonal hematopoiesis. DNMT3A mutations resulted in myeloid over lymphoid bias, and in expansion of immature myeloid progenitors primed toward megakaryocytic-erythroid fate. We observed dysregulated expression of lineage and leukemia stem cell markers. DNMT3A R882 led to preferential hypomethylation of polycomb repressive complex 2 targets and a specific sequence motif. Notably, the hypomethylation motif is enriched in binding motifs of key hematopoietic transcription factors, serving as a potential mechanistic link between DNMT3A R882 mutations and aberrant transcriptional phenotypes. Thus, single-cell multi-omics pave the road to defining the downstream consequences of mutations that drive human clonal mosaicism.

\section{INTRODUCTION}

Somatic mutations have been recently identified ubiquitously across healthy tissues, indicating the presence of acquired clonal mosaicisms ${ }^{1-6}$. These mutations are pervasive across tissues such as the blood $^{7-17}$, skin ${ }^{5}$, lung 2 and esophagus ${ }^{1,3}$, and their prevalence increases with physiological aging. Importantly, somatic mutations in these clonal outgrowths overlap with recurrent drivers of cancer (for example, DNMT3A, TP53, PIK3CA, and NOTCH1)1-5,8,18, suggesting that cancer may arise from pre-malignant clonal outgrowths. Nevertheless, mutated cells are morphologically and phenotypically similar to their wildtype counterparts. This limits the ability to define the downstream transcriptional or phenotypic impact that may drive clonal outgrowth, and therefore prior studies in primary human tissue have largely focused on genetic characterization of clonal mosaicism.

Clonal mosaicism within the hematopoietic system serves as an informative model for this phenomenon, as recurrent drivers of myeloid malignancies (for example, DNMT3A, TET2 and ASXL1 mutations) have been detected in individuals without overt hematologic abnormalities ${ }^{7-17}$. This state, termed clonal hematopoiesis $(\mathrm{CH})$, predisposes these individuals to an increased risk of developing myeloid malignancies, such as acute myeloid leukemias (AML) and myelodysplastic syndromes, and thus represents the earliest stages of neoplastic evolution ${ }^{8,19-21}$. Intriguingly, $\mathrm{CH}$ mutations also increase the risk of cardiovascular disease ${ }^{11}$ and progression of non-myeloid malignancies ${ }^{11,22,23}$, with early evidence supporting an aberrant immune microenvironment due to $\mathrm{CH}^{8,24-26}$. $\mathrm{CH}$ mutations have also been found in stem cell grafts, linked with idiopathic cytopenia in graft recipients ${ }^{27}$. $\mathrm{CH}$ mutations in certain genes (e.g. DNMT3A, TP53) endow a particularly strong fitness advantage in the context of stem cell transplantation, wherein the variant allele frequencies (VAF) markedly increase post-transplant compared to pre-transplant grafts ${ }^{28,29}$. These data suggest that certain $\mathrm{CH}$ mutations confer a particularly robust competitive advantage over non-neoplastic hematopoietic cells in stressed settings such as transplantation. 

available under aCC-BY-NC-ND 4.0 International license.

DNMT3A, which encodes a de novo DNA methyltransferase that catalyzes the methylation of cytosine bases in $\mathrm{CpG}$ dinucleotides, is by far the most frequently mutated gene in $\mathrm{CH}^{7-10}$. Consistently, DNMT3A mutations are considered an early event in $\mathrm{AML}^{7}$, and the hotspot variant at R882 constitute the majority of DNMT3A mutations in AML. The frequency of R882 variants is lower in $\mathrm{CH}$, suggesting that these variants are particularly prone to progressing to AML through clonal evolution ${ }^{12,30,31}$. In vitro and murine models have suggested that DNMT3A R882 (or the murine R878 homologous residue) mutations result in a differentiation block and increased self-renewal in the hematopoietic stem cells (HSCs) ${ }^{32-34}$. Biochemically, DNMT3A R882 variants may exhibit a dominant negative effect 35,36 , resulting in the reduction of methyltransferase activity ${ }^{36}$. However, the study of DNMT3A mutations directly in human samples has been largely limited to MDS or AML, where confounding cooccurrence of other genetic alterations is common. Thus, $\mathrm{CH}$ presents a unique setting to interrogate the molecular consequences of DNMT3A mutations in nonmalignant human hematopoiesis.

However, in $\mathrm{CH}$ as in other contexts of somatic mosaicism, mutated cells are admixed with wildtype cells ${ }^{12,31}$, limiting our ability to link genotype to phenotype using studies of bulk populations. Although recent fluidics methods for single-cell genotyping coupled with oligo-barcoded antibodies have begun to shed light on the phenotypic consequences of $\mathrm{CH}$ mutations ${ }^{37}$, these methods are limited to a small number of pre-defined cell surface markers. To overcome this limitation, we applied multi-omics singlecell sequencing to capture the mutational status of individual cells together with downstream epigenetic and transcriptional information ${ }^{38,39}$, thus enabling us to compare mutated cells with their wildtype counterparts from the same individuals, directly in primary human samples.

\section{RESULTS}

\section{Genotyping of DNMT3A mutations in single-cell RNA- seq of $\mathrm{CD}^{2} 4^{+}$cells of human clonal hematopoiesis}

As individuals with $\mathrm{CH}$ have normal blood production and thus meet no clinical criteria for assessments by bone marrow biopsy, progenitor-enriched samples with $\mathrm{CH}$ are scarce. However, we recently observed that $\mathrm{CH}$ is prevalent in patients with multiple myeloma (MM), and thus we interrogated a cohort of $136 \mathrm{MM}$ patients with $\mathrm{CH}$ identified in hematopoietic progenitor cells collected for autologous stem cell transplant while in remission ${ }^{40}$. Given the known strong phenotypic impact of DNMT3A R882 mutations, we focused on four samples with these mutations and sufficiently high VAFs of $>0.05$ (range:
0.09-0.34) to enable profiling of large numbers of mutated cells with single-cell RNA-sequencing (scRNAseq; see patient and sample data in Extended Data Fig. 1a) Supplementary Table 1). Notably, although $\mathrm{CH}$ mutations tend to have low VAFs, $\mathrm{CH}$ clones with higher VAFs have been frequently observed $8,10,41$. We further confirmed that no morphologic evidence of a myeloid neoplasm was seen in the bone marrow (Supplementary Table 1). Screening for additional mutations through a targeted myeloid panel ${ }^{40}$ showed only one additional mutation (patient $\mathrm{CH03}$ ), consisting of a clonal (VAF $=0.5$ ) heterozygous TET2 nonsense mutation, which therefore likely arose first in the course of clonal evolution and serves as a background mutation for both the DNMT3A R882 mutated and wildtype cells.

We isolated viable $\mathrm{CD}_{34}{ }^{+}$cells from these $\mathrm{CH}$ samples and performed Genotyping of Transcriptomes (GoT ${ }^{38}$ ), capturing scRNA-seq with targeted genotyping of the R882 codon (Fig. 1a). A total of 27,324 cells across $\mathrm{CH}$ samples were sequenced and included in the downstream analysis after quality filters (online methods, Extended Data Fig. 1b). Genotyping data were available for 6,430 cells of these 27,324 cells (23.5\%) through GoT (Extended Data Fig. 1a,c,d). Notably, to overcome the challenge of accurate genotyping of the lowly expressed DNMT3A gene, we performed deeper sequencing and further optimized the original GoT analysis pipeline (IronThrone ${ }^{38}$, see online methods). This optimization included integrating unique molecule identifier (UMI) consensus assembly ${ }^{42}$, resulting in enhanced precision, with increased number of cells correctly assigned with only mutant or wildtype UMIs in a species mixing experiment $\left(\mathrm{P}<10^{-10}\right.$, Fisher exact test, Extended Data Fig. 1e). We also filtered the GoT UMIs based on their presence in the $10 \mathrm{x}$ gene expression library to determine the threshold for the number of supporting reads (online methods, Extended Data Fig. 1f). Mutated $\mathrm{CD} 34^{+}$cell frequencies ranged from $13 \%$ to $50 \%$, comparable to the VAFs obtained through bulk sequencing of matched unsorted stem cell products (Extended Data Fig. 1a,c). Finally, to exclude additional genetic lesions, we performed copy number analysis with scRNA-seq data 43 and identified no significant chromosomal gains or losses (Extended Data Fig. 2a,b).

To chart the differentiation of $\mathrm{CD}_{34}{ }^{+}$progenitor cells in $\mathrm{CH}$, we integrated data across the samples ${ }^{44}$ and clustered based on transcriptomic data alone, agnostic to the genotyping information (Fig. 1b, Extended Data Fig. 3a, online methods). Consistent with clinical data indicating normal hematopoietic production, we identified the expected progenitor subtypes, using previously annotated progenitor identity markers (Fig. 
bioRxiv preprint doi: https://doi.org/10.1101/2022.01.14.476225; this version posted January 16, 2022. The copyright holder for this preprint (which was not certified by peer review) is the author/funder, who has granted bioRxiv a license to display the preprint in perpetuity. It is made available under aCC-BY-NC-ND 4.0 International license.

a
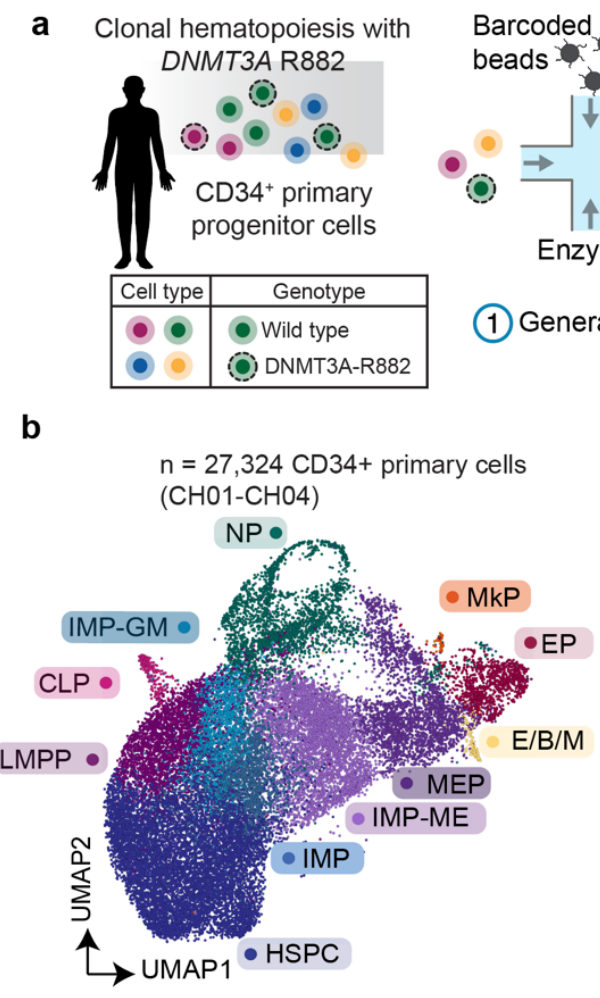

(4) Fragment and

(4) prepare library

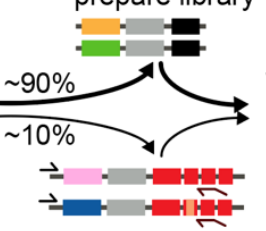

(3)

(2) Amplify with $1 \%$ gene-specific primer (3) locus of interest

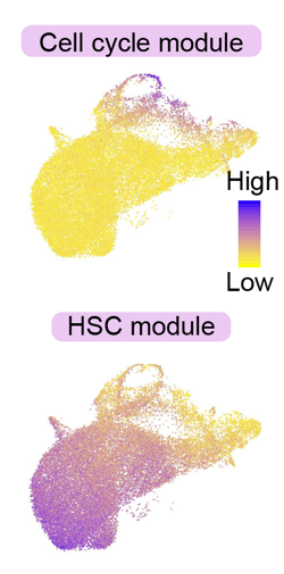

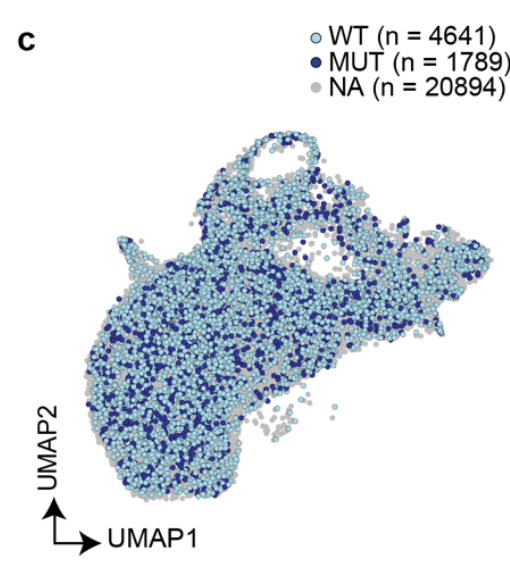

(5)

Sequence \& integrate transcriptomic \& genotyping data

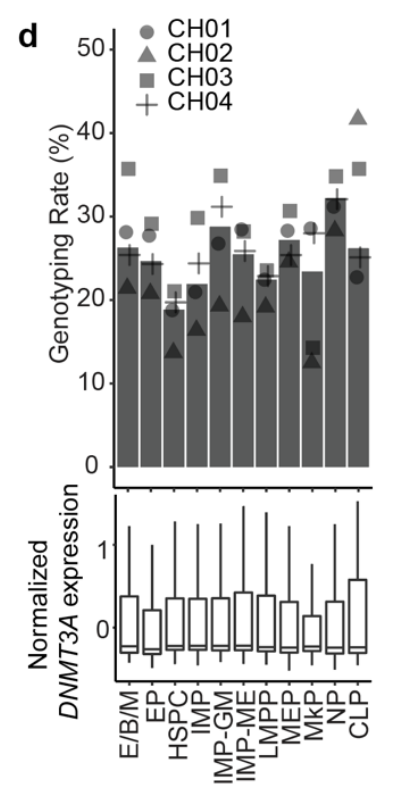

Figure 1. Genotyping of Transcriptomes demonstrates co-mingling of mutated and wildtype cells in DNMT3A R882clonal hematopoietic differentiation. a, Schematic of GoT workflow. UMI, unique molecular identifier; UTR, untranslated region. b, Uniform manifold approximation and projection (UMAP) of CD34+ cells ( $\mathrm{n}=27,324$ cells) from clonal hematopoiesis samples ( $\mathrm{n}=4$ individuals), overlaid with cluster assignment (left); projections of cell cycle gene module (top right) or uncommitted hematopoietic stem cell (HSC) associated gene module score (bottom right, Supplementary Table 2). c, UMAP of CD34 cells ( $\mathrm{n}=27,324$ cells) with projected mutation status assignment for WT ( $\mathrm{n}=4,641$ cells), DNMT3A R882 mutant (MUT; $\mathrm{n}=1,789$ cells) or unassigned (NA; $\mathrm{n}=20,894$ cells). $\mathbf{d}$, Percent of genotyped cells per cluster for all samples (bars) and for each patient sample (points) (top) and normalized gene expression of DNMT3A per cluster (bottom). HSPC, hematopoietic stem progenitor cells; IMP, immature myeloid progenitors; IMP-ME, megakaryocytic-erythroid biased IMP; IMP-GM, granulomonocytic biased IMP; LMPP, lympho-myeloid primed progenitors; CLP, common lymphoid progenitor; MEP, megakaryocyticerythroid progenitors; E/B/M, eosinophil, basophil, and mast cell progenitors; EP, erythroid progenitor; MkP, megakaryocytic progenitor; NP, neutrophil progenitor; WT, wildtype; MUT, mutant; NA, not assignable.

1b, Extended Data Fig. 3b-d, Supplementary Table 2) 45 . Furthermore, consistent with the fact that G-CSF mobilizes early stem and progenitor cells, we identified a large population of the earliest hematopoietic stem progenitor cells (HSPCs), as well as immature myeloid progenitor cells (IMPs), previously defined in a landmark scRNA-seq study ${ }^{45}$ as corresponding to the phenotypically-defined common myeloid progenitors (CMPs) and granulocyte-monocyte progenitors (GMPs). The high-throughput profiling by digital scRNA-seq enabled a higher resolution view of the IMPs, revealing a subcluster that exhibited markers of granulocytemonocyte differentiation (IMP-GM) and a subcluster that exhibited markers of megakaryocytic-erythroid differentiation (IMP-ME, Extended Data Fig. 4a,b). Having established the progenitor identities, we then projected the genotyping information onto the differentiation map (Fig. 1c, Extended Data Fig. 4c). No novel cell identities were formed by the DNMT3A mutations, consistent with the fact that patients with $\mathrm{CH}$ exhibit no overt peripheral blood count or morphologic abnormalities, Instead, we observed that mutated and wildtype cells co-mingled throughout (Fig. 1c, Extended Data Fig. 4c), highlighting the need for singlecell multi-omics to link genotypes with cellular phenotypes in $\mathrm{CH}$. Importantly, the genotyping efficiency was balanced across the progenitor subsets, mitigating potential technical biases (Fig. 1d, top), consistent with no significant difference in DNMT3A gene expression within the $\mathrm{CD} 34^{+}$cell subsets (Fig. 1d, bottom).

\section{DNMT3A-mutated cells show lineage biases at key differentiation junctures}

As previous data in murine and in vitro models have suggested that DNMT3A mutations may lead to a differentiation block ${ }^{46,47}$, we performed a differentiation pseudo-temporal (pseudotime) ordering analysis of the 
bioRxiv preprint doi: https://doi.org/10.1101/2022.01.14.476225; this version posted January 16, 2022. The copyright holder for this preprint (which was not certified by peer review) is the author/funder, who has granted bioRxiv a license to display the preprint in perpetuity. It is made available under aCC-BY-NC-ND 4.0 International license.
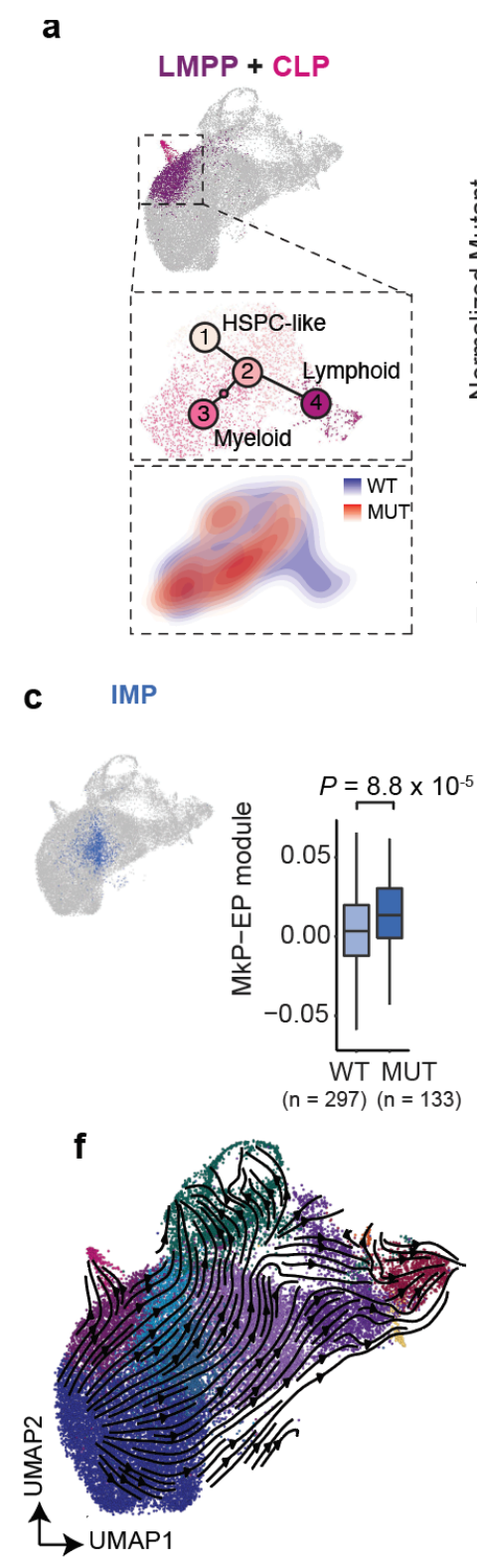

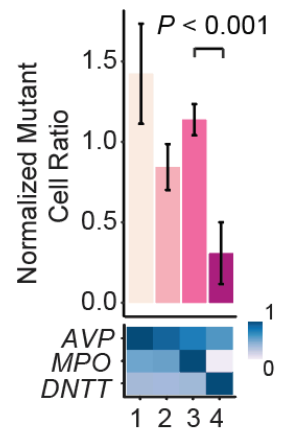

${ }^{*} \mathrm{CLP}: \mathrm{n}=71$ genotyped cells LMPP: $n=602$ genotyped cells

d IMP-ME + IMP-GM

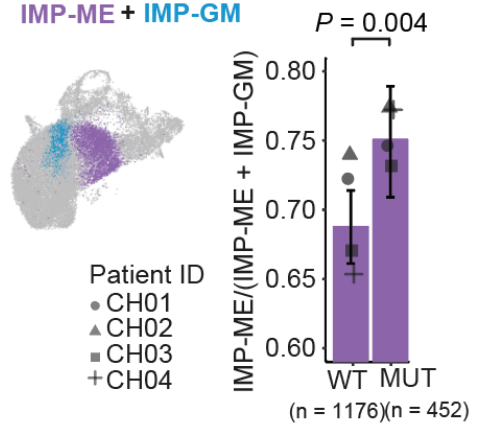

g

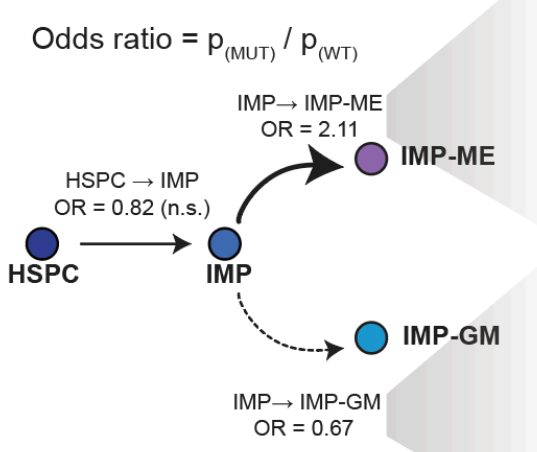

e
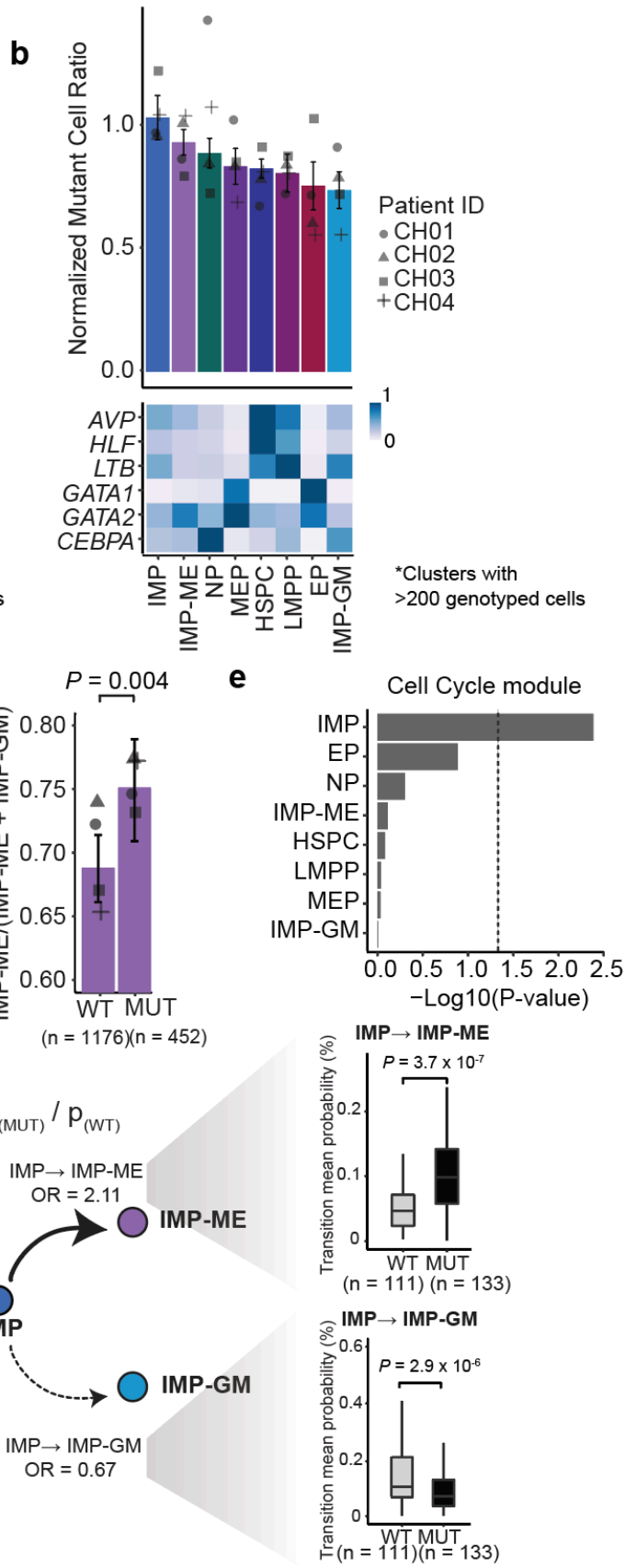

Figure 2. DNMT3A R882 mutated CH cells demonstrate distinct differentiation biases at key junctures. a, UMAP highlighting multi-lineage lympho-myeloid primed progenitors (LMPPs) and common lymphoid progenitors (CLPs); UMAP showing analytically isolated and re-clustered LMPPs and CLPs, showing branch point of divergence into myeloid versus lymphoid primed progenitors (left middle); UMAP showing the cell density of DNMT3A R882 MUT and WT cells (left bottom). The normalized frequency of mutant cells in subclusters for aggregate analysis of samples CH01-CH04 with mean \pm s.d. of 100 downsampling iterations to 1 genotyping UMI per cell (right, downsampling performed to control for potential greater ability to detect the mutant heterozygous allele in cells with higher DNMT3A expression, see online methods). The heatmap at the bottom depicts representative lineage-specific genes for individual clusters. P-value was calculated from likelihood ratio test of LMM with/without cluster identity. b, Normalized frequency of DNMT3A R882 mutant cells in progenitor subsets with at least 200 genotyped cells. Bars show aggregate analysis of samples $\mathrm{CH} 01-\mathrm{CH} 04$ with mean \pm s.d. of 100 downsampling iterations to 1 genotyping UMI per cell. Points represent mean of $n=100$ downsampling iterations for each sample. Heatmap depicts representative lineage-specific genes for individual progenitor subsets. c, Megakaryocytic-erythroid module scores in wildtype versus mutant IMPs (Supplementary Table 2). P-value was calculated from likelihood ratio test of LMM with/without mutation status. d, Fraction of IMP-ME cells out of all biased IMP (IMP-ME + IMP-GM) cells in wildtype versus DNMT3A R882 mutant populations. P-value was calculated from proportions test. e, Cell cycle module scores in wildtype versus mutant progenitor subsets (Supplementary Table 2). P-values were calculated from likelihood ratio test of LMM with/without mutation status. 
f, RNA velocity field vectors overlaid on UMAP, demonstrating differentiation trajectories computed via scVelo (online methods). g, Schematic representation of the transition probabilities between HSPCs and IMP subsets from samples CH01-CH04 (right). Odds ratios (OR) were calculated as the ratio between DNMT3A R882 MUT and WT transition probabilities, as measured using RNA velocity. Single cell mean IMP $\rightarrow$ IMP-ME or IMP $\rightarrow$ IMP-GM transition probabilities between wildtype or DNMT3A R882 mutant cells, inset. P-values were calculated from likelihood ratio test of LMM with/without mutation status (see Extended Data Fig. 6 for per-sample data).

GoT data ${ }^{48-50}$. We found no significant global difference between wildtype and mutated cells $(P=0.70$, linear mixed model, Extended Data Fig. 4d including per sample analysis, online methods), indicating that DNMT3A R882 mutations do not result in a significant global differentiation block in pre-cancerous human hematopoietic development. This finding is nonetheless consistent with findings in murine models, where even in the setting of homozygous Dnmt3a deletion, mutated cells do not exhibit self-renewal advantage in primary transplant experiments ${ }^{47}$, indicating that features of selfrenewal advantage may not be overtly obvious in steadystate hematopoiesis. Although we did not observe a global differentiation block, we hypothesized that the DNMT3A mutated cell frequencies may vary across certain progenitor identities. For example, as DNMT3A R882 mutations are more frequently associated with myeloid rather than lymphoid neoplasms, we tested whether mutated cells may demonstrate a lineage bias toward myeloid versus lymphoid differentiation by examining lympho-myeloid primed progenitors (LMPP) and common lymphoid progenitors (CLP). Consistent with frequency biases seen in murine models for DNMT3A mutations ${ }^{51}$, mutated cells were enriched in myeloid biased cells versus early lymphoid progenitors $(\mathrm{P}<0.001$, linear mixed model, Fig. 2a). Moreover, these data are also consistent with previous results obtained with bulk, sorted populations from a DNMT3A I780T CH sample, which showed a lower VAF in mutated cell frequency in mature lymphoid cells (e.g. NK cells, B cells), compared to those in myeloid progenitor and mature cells 52 .

To identify differentiation biases more broadly in DNMT3A-mutated $\mathrm{CH}$, we evaluated the mutated cell frequencies across the different prevalent progenitor cell types ( $>200$ genotyped cells). Of note, as cells may display variable expression of DNMT3A itself, we performed amplicon UMI down-sampling to exclude sampling biases given the heterozygosity of the mutated allele as a potential confounder for observed differences in mutated cell frequencies ${ }^{38}$. We observed that across samples, mutated cells were enriched in IMPs compared to the earliest HSPCs $(\mathrm{P}<0.001$, linear mixed model, Fig. 2b). Mutated IMPs also displayed an ME bias with an increase in the expression of an MkP-EP gene $\operatorname{set}^{53}(\mathrm{P}=$ $8.8 \times 10^{-5}$, linear mixed model, Fig. 2c, Supplementary Table 2, online methods), consistent with an increase in the proportion of IMP-ME to IMP-GM in mutant compared to wildtype cells $(\mathrm{P}=0.004$, proportions test, odds ratio of 1.38 (1.08 - 1.76), Fig. 2d). These data are in line with evidence of subtle erythroid abnormalities observed in $\mathrm{CH}$ via routine clinical assays (e.g. elevated red cell distribution width (RDW)) $)^{21}$, and with our recent demonstration of increased HSC erythroid priming in a Dnmt3a knock-out murine model ${ }^{54}$.

Increased mutated cell frequency in a specific progenitor subtype can result from cell-type specific elevated proliferation ${ }^{38}$. We therefore first compared the expression of cell cycle genes ${ }^{55}$ between mutated and wildtype progenitors and found a modest increase in cell cycle gene expression only in mutated IMPs $(\mathrm{P}=4.1 \times 10$ 3, linear mixed model, Fig. 2e, Extended Data Fig. 5a). Alternatively, increased mutated cell frequency in a given progenitor subtype, may stem from a change in transition rates into this cell state. To explore this hypothesis, we measured transition probabilities between progenitor subtypes with RNA velocity (online methods) ${ }^{56,57}$. The overall RNA velocity measurements demonstrated that these mobilized $\mathrm{CD} 4^{+}$cells follow the expected differentiation trajectories as described in normal human bone marrow hematopoiesis ${ }^{33,58}$ (Fig. 2f). Consistent with the hypothesis that transition rates contribute to the observed differentiation biases, we identified that the transition probability of mutated IMPs to become IMP-MEs was higher compared to that of wildtype cells $\left(\mathrm{P}=3.7 \times 10^{-7}\right.$, linear mixed model, Fig. 2g, see Extended Data Fig. 6a for per sample comparison), whereas the transition probability of mutated IMPs to IMP-GMs was diminished $(\mathrm{P}=2.9 \times 10$ 6 , linear mixed model, Extended Fig. 6b). These analyses thus orthogonally confirmed ME-biased differentiation of DNMT3A-mutated CD34+ human progenitors, as was also revealed by the gene set expression analysis (Fig. 1c).

Gene expression changes in DNMT3A mutated cells include leukemia stem cell genes, and are linked to proinflammatory signatures and putative dysregulated transcription factor activity

To identify the transcriptional dysregulation that may underlie the observed differentiation biases, we performed differential gene expression analysis between mutated and wildtype progenitors within each progenitor cell type. Differential expression (DE) analysis of mutated versus wildtype HSPCs revealed 88 dysregulated genes (Fig. 3a, 68-122 differentially 
a HSPC -

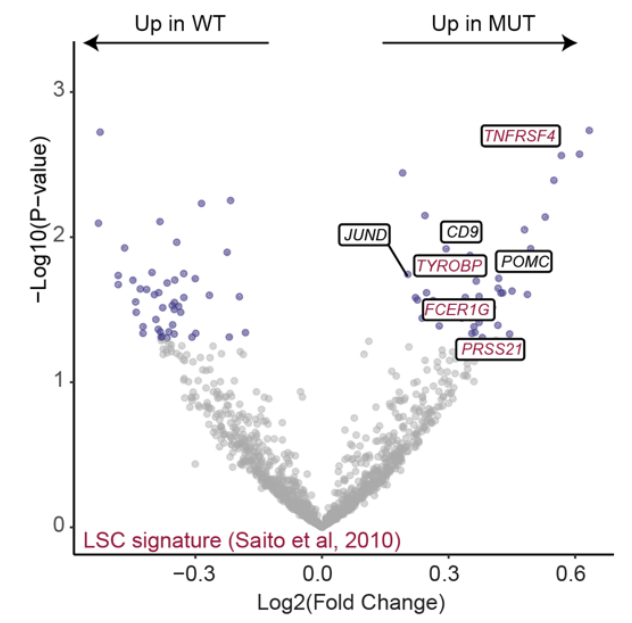

b

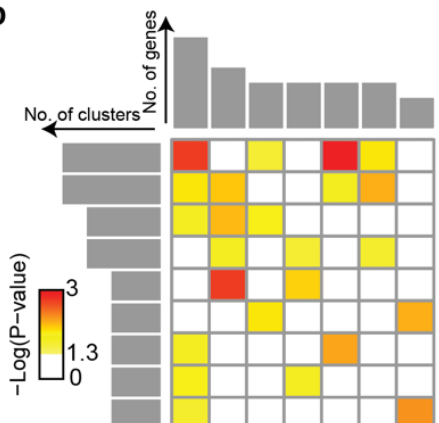

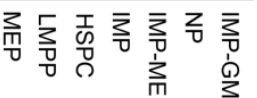

PRSS21 MYC, MAX, REST, AP-1, STAT5A

CLEC11A REST, RORA, AHR, ARNT, NFE2L1, ARID5B, NF1 POMC CREB1, STAT3

C1QTNF4 CREB1, RORA, YY1, ZBTB6

JUND $\quad$ CREB1, NFKB1, REST, SP1, CEBPB

HLA-DMA MYC, MAX, CREB1, IRF7, TBP, SRY

SERPINB1 STAT3, TBP, CUX1, NFATC3, POU2F1

HEMGN IRF7, NFKB1, RUNX1, ZEB1, FOXJ2

d
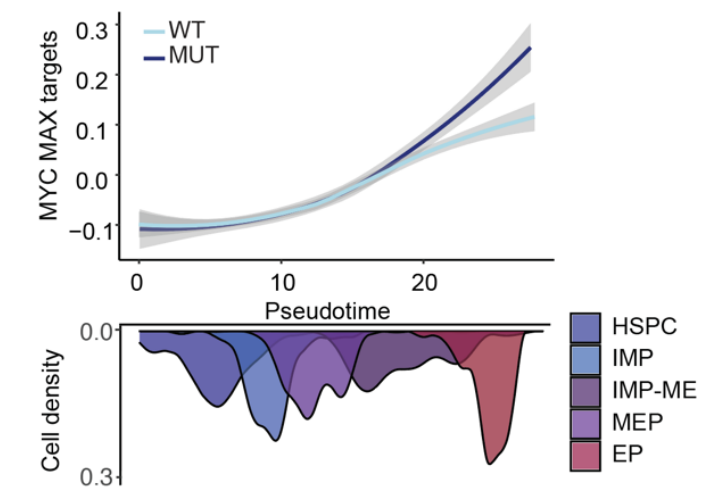

d

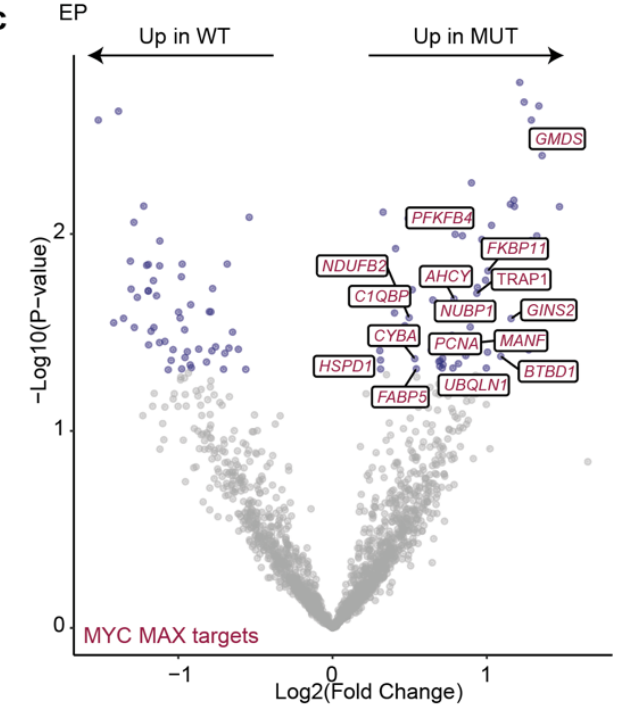

Figure 3. Differential gene expression analysis between mutated and wildtype cells reveals markers of lineage aberrancies and dysregulated MYC activity. a, Differentially expressed (DE) genes between DNMT3A R882 mutant and wildtype hematopoietic stem progenitor cells (HSPC) via permutation test (online methods). Genes highlighted in red represent DE genes overlapping with 58 genes upregulated on acute myeloid leukemia stem cells (LSC) compared to normal HSCs ( $\mathrm{P}=9.3$ $\mathrm{x} 10^{-5}$ ). P-value was calculated by hypergeometric test. b, Heatmap of upregulated genes in DNMT3A mutant cells compared to wildtype cells, in at least two cell clusters ( $\mathrm{P}<0.05$, permutation test). Histograms show numbers of upregulated genes in each cluster (top) and numbers of clusters per upregulated gene (left). Next to the genes are listed putative TFs (TRANSFAC) with black indicating the TFs that overlap for more than one recurrent DE gene. c, Differentially expressed genes between $D N M T 3 A$ R882 mutant and wildtype EPs via permutation test. Pathway enrichment of MSigDB CGP gene sets shows enrichment of Benporath MYC MAX targets (FDR-adjusted P-value = 0.01) and Coller MYC targets (FDR-adjusted P-value = 0.01, see Supplementary Table 4 for complete gene set enrichment results against the MSigDB CGP dataset). P-values were calculated from hypergeometric test with FDR (Benjamini-Hochberg) correction. d, Local regression of normalized expression levels as a function of pseudotime of MYC/MAX targets (differentially upregulated in Fig. 3c) for WT and DNMT3A R882 mutant (MUT) cells. Shading denotes $95 \%$ confidence interval. Histogram shows cell density of clusters included in the analysis, ordered by pseudotime.

expressed genes in each progenitor subset, see Supplementary Table 3 for results for each progenitor subset; batch-aware permutation test where mutated and wildtype labels are permuted only within the same sample, see online methods). Of note, to ensure that the analysis was not dominated by a single sample, we down-sampled the number of mutated and wildtype cells from each sample to maintain equal representation in the progenitor subset DE analysis. To test the robustness of our approach further, we also determined DE by an alternative linear mixed model framework, in which we explicitly modeled samples as a random effect variable, and identified a high degree of concordance between the two statistical frameworks (Extended Data Fig. 7a, Supplementary Table 3, online methods).

DE genes included, for example, the upregulation of $C D 9$ in the early mutated HSPCs (Fig. 3a, Supplementary 

available under aCC-BY-NC-ND 4.0 International license.

Table 3). CD9 expression is closely linked with megakaryocytic-priming 59,60 and platelet activation ${ }^{61-63}$, thus providing further support for the ME bias of DNMT3A mutated progenitors. These data are also in line with a lower degree of thrombocytopenia observed in patients with DNMT3A mutated versus wildtype AML ${ }^{64,65}$ and thrombocytosis in a murine model of this mutation 66 . We further observed an enrichment of genes previously associated with leukemia stem cells (LSCs) ${ }^{67}$ in mutated HSPCs, including PRSS21, FCER1G, TYROBP, and TNFRSF4, mapping these dysregulated genes to the nascent neoplastic process $\left(\mathrm{P}=9.3 \times 10^{-5}\right.$, hypergeometric test, Fig. 3a, Supplementary Table 3). FCER1G, TYROBP and TNFRSF4, are known to be involved in proinflammatory signaling68-76, consistent with previous reports suggesting that $\mathrm{CH}$ clones display enhanced proinflammatory signatures $24,26,41,77-81$. In another example, we identified upregulation of the prosurvival oncogene PIM2, downstream of STAT signaling, in mutated LMPPs, recently implicated as a target for eradicating chemotherapy-resistant chronic myeloid leukemia stem cells 82 (Supplementary Table 3).

Nine genes were upregulated in more than one progenitor subset (Fig. 3b, Supplementary Table 3). This analysis highlighted mediators of cell-to-cell interactions, such as a regulator of the inflammatory network C1QTNF483,84. We also identified CLEC11A (also known as stem cell growth factor (SCGF)), which has been implicated as a hematopoietic growth factor ${ }^{85,86}$, including in the setting of hematopoietic stress such as irradiation and transplantation ${ }^{85,87}$. This finding is consistent with published murine data showing a 6.75fold increase of Clec11a in transplanted Dnmt3a KO cells compared to wildtype cells ${ }^{88}$. Thus, overexpression of CLEC11A by DNMT3A-mutated progenitors may provide a potential mechanism for marked expansion of $\mathrm{CH}$ clones upon transplantation $28,29,89-93$. Genes upregulated in more than one progenitor subset were associated with putative transcription factors ${ }^{94}$, identifying recurring TFs (highlighted in black, Fig. 3b), including MYC and its cofactor MAX, as well as the inflammatory NFKB and STAT transcription factors and interferon regulatory factor IRF7, consistent with proinflammatory networks in $\mathrm{CH}$ clones $24,26,77,80,81$.

To more broadly identify dysregulated pathways, we performed a gene set enrichment analysis of the differentially upregulated genes (Fig. 3c, Supplementary Table 4) $)^{95,96}$. The top significantly enriched pathways (FDR $<0.2$ ) included MYC targets in the mutated erythroid progenitors (FDR-adjusted $\mathrm{P}=$ 0.01 , Fig. 3c). Notably, we observed the enrichment of two independent MYC target gene sets, including a MYC signature that was downregulated with monocytic differentiation in an HSPC differentiation cell line model ${ }^{97,98}$. Consistently, MYC has been demonstrated to be a critical factor specifically for erythropoiesis $99-101$, and may thus contribute to the observed ME bias (Fig. $\mathbf{2 c , d , g ) . ~ O f ~ i n t e r e s t , ~ D N M T 3 A ~ m u t a t i o n ~ d r i v e n ~ M Y C ~}$ target expression increased during differentiation along the erythroid lineage (Fig. 3d), despite no increase in MYC gene expression itself in the mutated progenitors (Extended Data Fig. 7b), suggesting that its transcriptional output as a transcription factor is differentially increased in mutated cells. Other enriched pathways included targets of cell cycle regulator E2F in LMPPs (FDR-adjusted P $=0.057$, Supplementary Table 4). Altogether, these findings suggest a focused dysregulation in TF activity that may orchestrate the observed lineage and transcriptional perturbations in the premalignant stages of hematopoietic neoplasia.

\section{Single-cell multi-omics integrating somatic genotyping, methylome, and transcriptome profiling reveals patterns of DNMT3A mutation hypomethylation}

To directly decipher the underlying link between mutated DNMT3A-induced DNA hypomethylation and the observed altered transcriptional regulatory networks in $\mathrm{CH}$, we profiled $\mathrm{CD} 34^{+}$progenitors from the same individuals (from samples $\mathrm{CHO} 2$ and $\mathrm{CHO} 4$ where additional material was available) with multi-modality single-cell sequencing capturing DNA methylation (DNAme) ${ }^{102}$, scRNA-seq (Smart-seq2103), and targeted DNMT3A genotyping 39 ( $\mathrm{n}=528$ cells after quality filtering, Fig. 4a,b, Extended Data Fig. 8a-c, online methods). As expected, these scRNA-seq data identified the major progenitor identities as those demonstrated by the $10 \mathrm{x}$ platform, albeit at a lower resolution given fewer cells (Fig. 4b, left, Extended Data Fig. 8b). Of these 528 cells, genotyping data were available for 441 cells (Fig. 4b, right, 84\% cells genotyped). This multimodal profiling uniquely enabled us to compare the methylation status of mutated and wildtype cells from the same individuals, showing a decrease in DNAme in $\mathrm{CpG}$ islands even in this relatively heterogeneous CD34+ population (CGI, P = 5.72 x 10-3, linear mixed model, Fig. 4c), consistent with the finding that DNMT3A mutated AMLs have lower methylation of CGI compared to DNMT3A wildtype AMLs ${ }^{104}$. While enhancers have been demonstrated to be particularly impacted by DNMT3A loss in the setting of $\mathrm{AML}^{105}$, these relatively $\mathrm{CpG}$-poor regions have lower coverage in standard enzymatic methyl-seq (EM-seq) ${ }^{106}$ or reduced representation bisulfite sequencing (RRBS) with a single restriction enzyme Msp1. We therefore increased the capture of enhancer regions through double restriction-enzyme Msp1 and HaeIII digestion ${ }^{107}$ and identified marked 
bioRxiv preprint doi: https://doi.org/10.1101/2022.01.14.476225; this version posted January 16, 2022. The copyright holder for this preprint (which was not certified by peer review) is the author/funder, who has granted bioRxiv a license to display the preprint in perpetuity. It is made available under aCC-BY-NC-ND 4.0 International license.

a

Multi-omics single cell sequencing

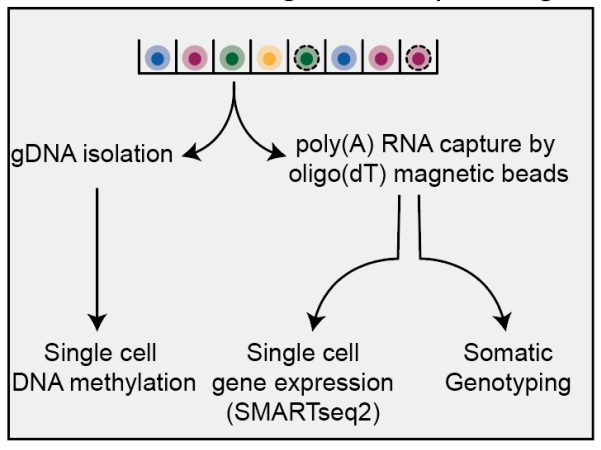

C

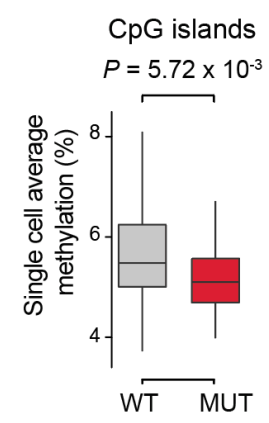

d

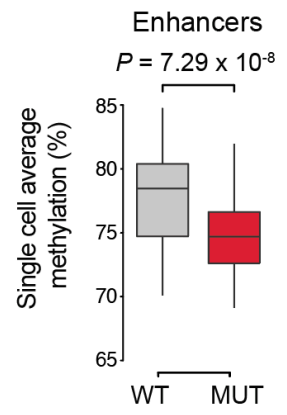

f Preferentially hypo-methylated ChIP-seq peaks (ENCODE)

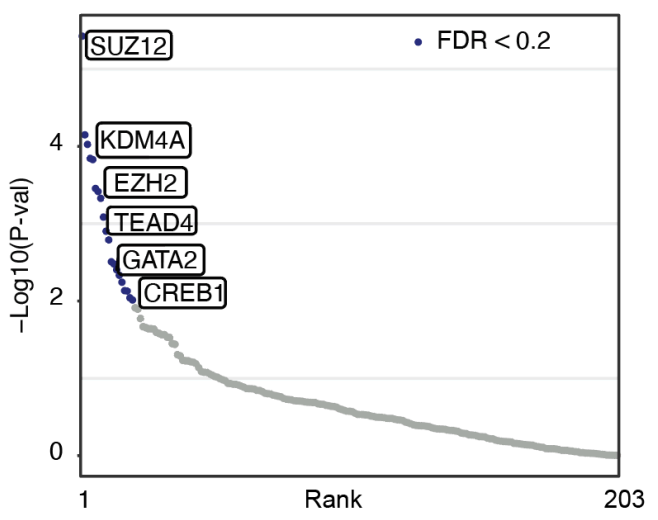

b

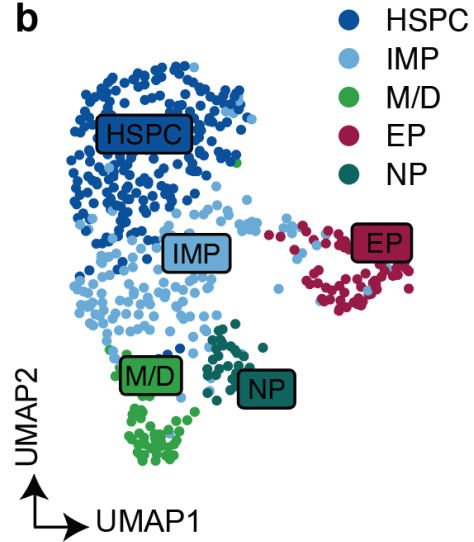

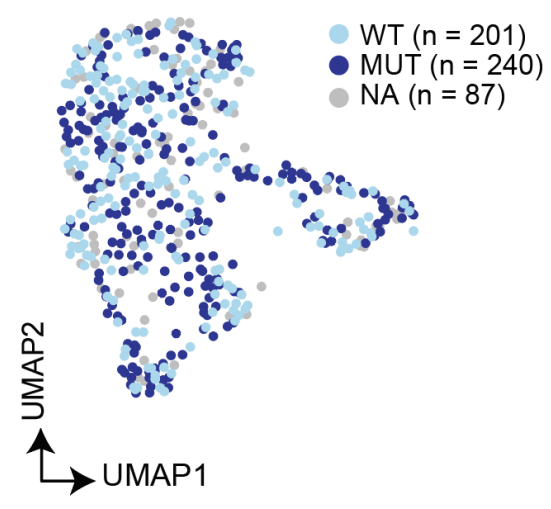

TSS +/- 1 kbp DMR

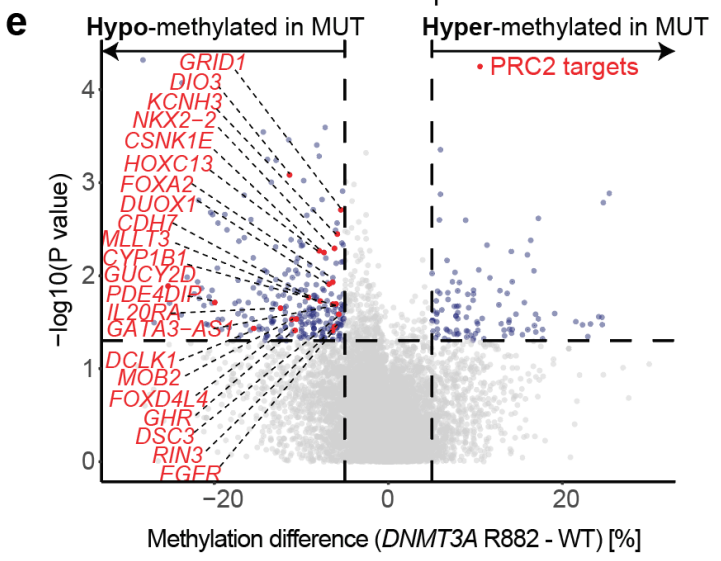

g

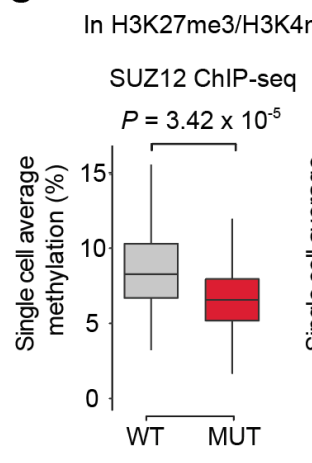

h

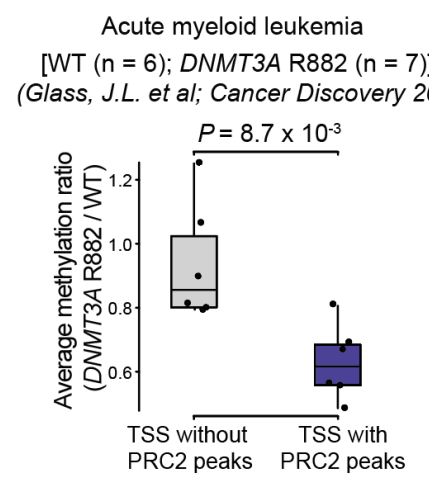

Figure 4. DNMT3A R882 promotes selective hypomethylation of PRC2 targets in human hematopoiesis. a, Schematic representation of the single-cell multi-omics platform that captures methylome, transcriptome, and somatic genotype status. $\mathbf{b}$, UMAP dimensionality reduction ( $\mathrm{n}=528$ cells) showing the assigned progenitor identities (left) or the assigned genotype (right) from available samples $\mathrm{CHO} 2$ and $\mathrm{CH04}$. (c-d) Average single cell methylation at $\mathrm{CpG}$ islands c, and enhancers d, from double digest experiments (online methods). P-values from likelihood ratio test of LMM with/without mutation status. e, Differentially methylated promoters between wildtype and DNMT3A R882 mutant hematopoietic progenitors. P-values from generalized linear model (GLM) to account for global hypomethylation in DNMT3A mutated cells and identify regions of preferential hypomethylation (online methods). Red dots indicate significantly hypomethylated Benporath PRC2 and EED target genes (MSigDB C2: CGP gene sets). f, Differentially hypomethylated ChIP-seq peaks (ENCODE hg38 Tf clusters) ranked by P-value. Pvalues from a GLM to account for global hypomethylation in DNMT3A mutated cells and identify regions of preferential hypomethylation. g, Single cell average methylation at ChIP-seq peaks (ENCODE hg38 Tf clusters intersected with bivalent peaks (H3K27me3, H3K4me3) from human CD34+ hematopoietic progenitor cells) for either SUZ12 (left) or EZH2 (right). P-values from likelihood ratio test of LMM with/without mutation status. h, Comparison of AML samples with/without DNMT3A R882 showing DNMT3A mutant-to-wildtype ratio of methylation at TSS overlapping PRC2 ChIP-seq peaks or non-overlapping CpG 
rich TSS as control. P-value from two-sided Wilcoxon rank sum test. HSPC, hematopoietic stem progenitor cells; IMP, immature myeloid progenitor; NP, neutrophil progenitor; M/D, monocytic/dendritic cell progenitors; EP, erythroid progenitor; WT, wildtype; MUT, mutant; NA, not assignable.

hypomethylation of enhancer regions ${ }^{108}\left(\mathrm{P}=7.29 \times 10^{-8}\right.$, linear mixed model, Fig. $4 \mathbf{d})$ as well as global hypomethylation in DNMT3A R882 cells compared to wildtype cells $\left(\mathrm{P}=2.92 \times 10^{-3}\right.$, linear mixed model, Extended Data Fig. 8c-d, online methods). Thus, we demonstrated that the methylation of regulatory regions is affected by DNMT3A R882 mutations in human $\mathrm{CH}$. Interestingly, prior in vitro studies suggested that $\mathrm{CpH}$ sites may be hypermethylated in DNMT3A R882H. Our data revealed no significant difference, and an opposite trend (Extended Data Fig. 8e), further highlighting the significance of examining primary human cells.

Differentially methylated regions (DMR) analysis identified 269 promoters to be significantly hypomethylated considering the observed global hypomethylation $(\mathrm{P}<0.05$ and at least $5 \%$ loss in methylation, Fig. 4e, Extended Data Fig. 8f, Supplementary Table 5, see online methods for statistical modeling to identify promoters with preferential hypomethylation that explicitly models samples as a variable). Gene set enrichment analysis of these genes identified enrichment of targets of the PRC2 (FDR-adjusted P < 0.2, GSEA with MSigDB C2: CGP gene set, Fig. 4e, Supplementary Table 6, online methods). As an orthogonal approach, we performed differential methylation analysis of chromatin immunoprecipitation sequencing (ChIP-seq) peaks (ENCODE database ${ }^{109}$ ) that overlap with TSS regions. This approach also identified the targets of PRC2 components SUZ12 and EZH2 to be differentially hypomethylated (Fig. 4f), as well as that of GATA2, involved in ME differentiation. As ENCODE ChIPseq tracks reflect aggregation across several cell types, we validated that preferential hypomethylation specifically impacted regions marked by H3K27me3, H3K4me3 bivalency in human hematopoietic progenitors, by intersecting the ENCODE ChIP-seq tracks with bivalent peaks in $\mathrm{CD}^{2} 4^{+}$cells ${ }^{110}$ (Fig. 4g, Supplementary Table 7, for per-sample data see Extended Data Fig. 8g). This finding is consistent with previous data showing that germline gain-of-function mutations in DNMT3A result in the reciprocal hypermethylation of PRC2 targets, leading to premature differentiation programs ${ }^{111}$. Furthermore, PRC2 targets exhibit significant overlap with previously reported methylation canyons, shown to undergo preferential hypomethylation upon Dnmt3a loss ${ }^{112}$ (98\% of canyons harbored a PRC2 target compared with $16 \%$ of canyons harboring peaks of a size-matched set of random genomic intervals, $\mathrm{P}<10^{-10}$, Fisher exact test) ${ }^{113}$. Notably, while gene expression changes in PRC2 targets were not observed between mutated and wildtype cells from the GoT data $(\mathrm{P}=0.42$, linear mixed model, Extended Data Fig. 8h), this may be expected given that PRC2-repressed genes that gain DNA methylation may only switch between different silencing states. Nonetheless, DNA methylation of PRC2 targets has been shown to reinforce gene silencing114-116, and thus mutated DNMT3A mediated hypomethylation of PRC2 targets may poise mutated progenitors to aberrant reactivation of stem cell maintainers, as seen in a PRC2 deficient mouse model ${ }^{117}$.

Finally, to determine whether $\mathrm{CH}$ hypomethylation of PRC2 targets persists through progression to AML, we compared the methylation status of PRC2 targets (online methods) between DNMT3A R882 mutated AML $(\mathrm{n}=7)$ and DNMT3A wildtype AML ( $\mathrm{n}=6$, both groups with NPM1 mutations ${ }^{105}$, Supplementary Table 8). We found that compared with DNMT3A wildtype AML, DNMT3A R882 mutated AML demonstrated preferential hypomethylation at promoters of PRC2 targets compared to promoters with similar $\mathrm{CpG}$ content $(\mathrm{P}=$ 0.0087, online methods, Fig. 4h, Extended Data Fig. 8i). To determine whether the preferential hypomethylation of PRC2 targets may be robust against various cooccurring mutations, we compared the methylation rates of PRC2 targets in DNMT3A wildtype $(\mathrm{n}=122)$ versus DNMT3A R882 mutated AML $(\mathrm{n}=9)$ with heterogeneous mutation status from The Cancer Genome Atlas (TCGA) 118 and identified similar results as observed in the NPM1-mutated AML (Extended Data Fig. 8j). These results demonstrated that mutated DNMT3A-mediated hypomethylation of PRC2 targets is maintained through evolution to AML, further supporting it as a potential mechanism for enhanced self-renewal, from clonal hematopoiesis to frank malignancy.

\section{DNMT3A R882 displays differential methyltransferase activity as a function of CpG flanking sequence}

We hypothesized that mutated DNMT3A R882 may further display differential methyltransferase activity, depending on the flanking sequence context of the $\mathrm{CpG}$ dinucleotide 119,120 . Indeed, CpGs within DMRs defined CpG motifs that are particularly hypomethylated (disfavored) in mutated versus wildtype human CD34+ cells (online methods, Fig. 5a, Extended Data Fig. 9a). Of note, CpGpT was particularly associated with hypomethylation (Fig. 5a, Extended Data Fig. 9a), consistent with in vitro enzymatic studies of DNMT3A R882 variants 119,120 (Extended Data Fig. 9b,c). Importantly, this CpG flanking motif was enriched in the 
binding motifs of specific TFs expressed in hematopoietic progenitors (Fig. 5b). These included key regulators of hematopoiesis such as MYC/MAX, whose activities are known to be negatively impacted by DNA methylation of their binding motifs ${ }^{121,122}$, and were found to have increased target expression in mutated cells (Fig. 3c,d). Other key transcription regulators included HIF1A (and its cofactor ARNT), whose binding is facilitated by demethylation of the binding motif ${ }^{123}$; HIF1A/ARNT are critical factors for HSC quiescence, through maintenance of the anaerobic glycolysisdependent metabolic activity in the bone marrow niche ${ }^{124-130}$. USF1/2 were also among the highlighted TFs, which have been shown to regulate chromatin architecture in erythroid differentiation and the betaglobin locus ${ }^{131,132}$. In further support for a model in which preferential hypomethylation of the specific sequence motif underlies transcriptional dysregulation, we observed enrichment of the hypomethylated $\mathrm{CpG}$ flanking sequence in regions surrounding genes upregulated in mutated HSPCs and EPs (Fig. 5c, Extended Data Fig. 9d-f).

To validate the impact of mutated DNMT3A on TF activation, we collected Lin-, c-Kit+ hematopoietic stem and progenitor cells from mice with and without Dnmt3a $\mathrm{R} 878 \mathrm{H}$ (the murine R882H equivalent; no. of mice $=3$ in each cohort) ${ }^{51}$. While recent progress has been made in single-cell chromatin binding assays ${ }^{133-135}$, the ability to determine the weaker signal of TF binding in single cells remains a challenge. We therefore performed a chromatin accessibility assay, shown to be a reliable surrogate for determining TF activity ${ }^{136}$, on single nuclei ( $n=46,496$ cells, Fig. 5d, Extended Data Fig. 10a-d). Confirming our findings in human $\mathrm{CH}$, we found that the accessibility of the DNMT3A R882-specific hypomethylated motif was increased in R878H cells, across clusters, including in HSPCs, and particularly in EPs (Fig. 5e,f, Extended Data Fig. 10e-g), whereas shuffled versions of the hypomethylated motif, with or without a $\mathrm{CpG}$, displayed lower difference in accessibility between mutated and wildtype progenitors. Candidate TFs with high similarities scores in their binding motif with the hypomethylated motif, including MYC/MAX, HIF1A/ARNT, USF1/2, displayed enhanced accessibility in R878H compared with wildtype progenitors, across multiple progenitor subsets (Fig. 5f, Extended Data Fig. 10g). The myeloid progenitors were particularly impacted, whereas the lymphoid progenitors showed little to no significant difference in accessibility for these TF binding motifs (Extended Data Fig. 10g), suggesting overactivity of these TFs may play a role in the myeloid differentiation bias. While Dnmt3a R878H HSPCs displayed a more modest increase in chromatin accessibility, this may be due to the global open chromatin in stem cells reducing the ability to measure specific enrichments 137,138 . Overall, as chromatin accessibility has been demonstrated to accurately reflect TF activity ${ }^{136}$, these data provided further evidence for the model in which the DNMT3A mutation enhances the activity of TFs whose binding motifs are prone to hypomethylation through enrichment in the hypomethylated sequence motif. This model then provides the basis of enhanced MYC/MAX target gene expression in the DNMT3A mutated cells observed in the GoT data (Fig. 3c,d), despite no expression increase in the $M Y C$ gene itself (Extended Data Fig. 7b). With respect to PRC2 targets, although hypomethylation of PRC2 target genes were observed, we observed no differential increase in expression in mutated cells (Extended Data Fig. 8h) and no enhanced accessibility of PRC2 targets in the mutated cells from mouse snATAC-seq data (Extended Data Fig. 10h).

As further confirmation of our proposed model, we found that HIF1A/ARNT and MYC/MAX binding motifs were hypomethylated in $\mathrm{CH}$ mutated cells compared to wildtype progenitors in the single-cell multi-omics data $\left(\mathrm{P}=2.7 \times 10^{-4}\right.$ and $\mathrm{P}=1.7 \times 10^{-2}$, respectively, linear mixed model, Fig. 5g,h). Moreover, as MYC targets were upregulated in $\mathrm{CH}$ mutated cells in the GoT data, we leveraged our single-cell multi-omics approach to directly link the expression of MYC/MAX targets with the level of DNA methylation of MYC/MAX target promoters within the same cells (see online methods). Indeed, the expression of MYC/MAX target genes was negatively correlated with mean methylation of their binding sites $\left(\mathrm{P}=3.2 \times 10^{-18}\right.$, generalized linear model, Fig. 5i $)$, consistent with prior studies indicating that hypomethylation of binding motifs enhances MYC binding121,122,139,140. Thus, our single-cell multi-omics profiling provides a potential model for the observed transcriptional aberration in human DNMT3A mutated $\mathrm{CH}$, supporting enhanced fitness of DNMT3A mutated cells via selective hypomethylation of key hematopoietic TF binding motifs.

\section{DNMT3A-mutated CH bone marrow sample corroborates results from stem cell graft $\mathrm{CH}$ samples}

To confirm that the findings we observed in the $\mathrm{CH}$ samples were generalizable to $\mathrm{CH}$ not exposed to G-CSF or prior chemotherapy, we obtained a bone marrow sample from a patient without any underlying hematologic disorders with a DNMT3A R882H mutation (CH05). We sorted for CD34+ cells and performed GoT as we had done for CH01-CH04 samples ( $n=5,770$ cells). Although a low genotyping efficiency limited the comparisons between mutated and wildtype cells within the same sample ( $n=687$ genotyped cells), this sample 
bioRxiv preprint doi: https://doi.org/10.1101/2022.01.14.476225; this version posted January 16, 2022. The copyright holder for this preprint (which was not certified by peer review) is the author/funder, who has granted bioRxiv a license to display the preprint in perpetuity. It is made available under aCC-BY-NC-ND 4.0 International license.

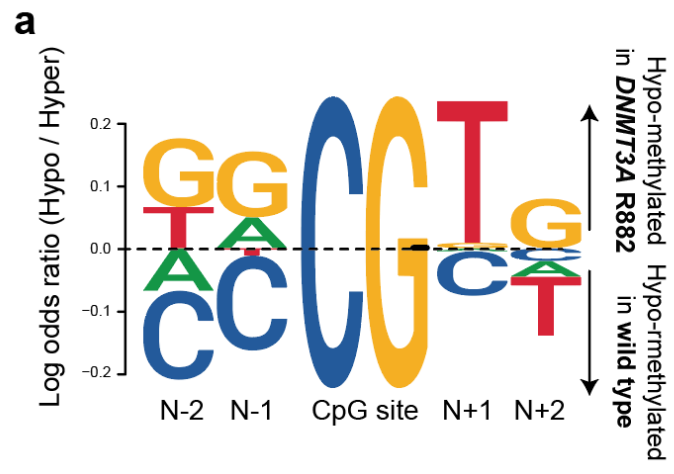

c

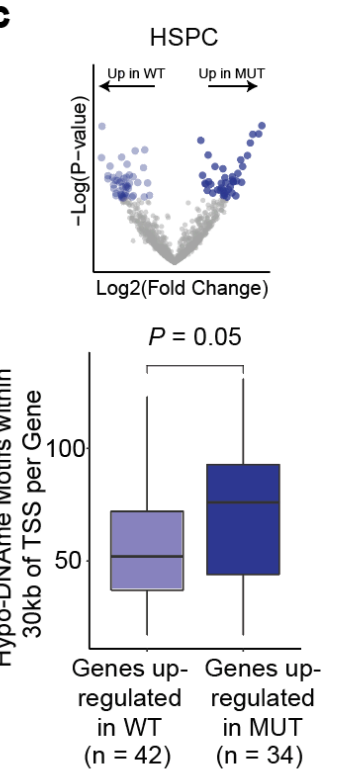

b

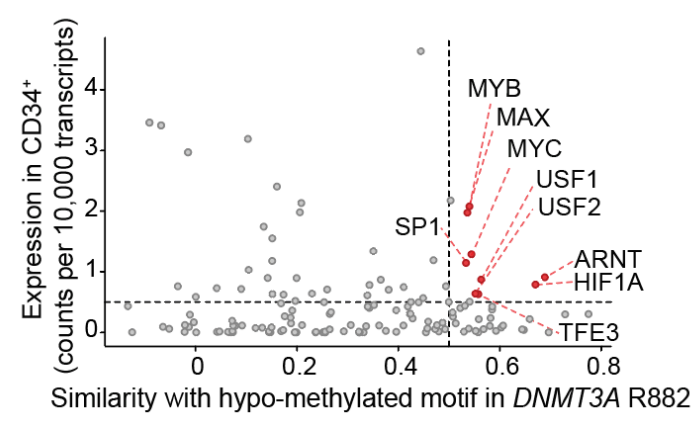

d

ScATAC: Mouse hematopoietic progenitors $\left(\mathrm{Lin}-\mathrm{Kit}^{+}\right)$
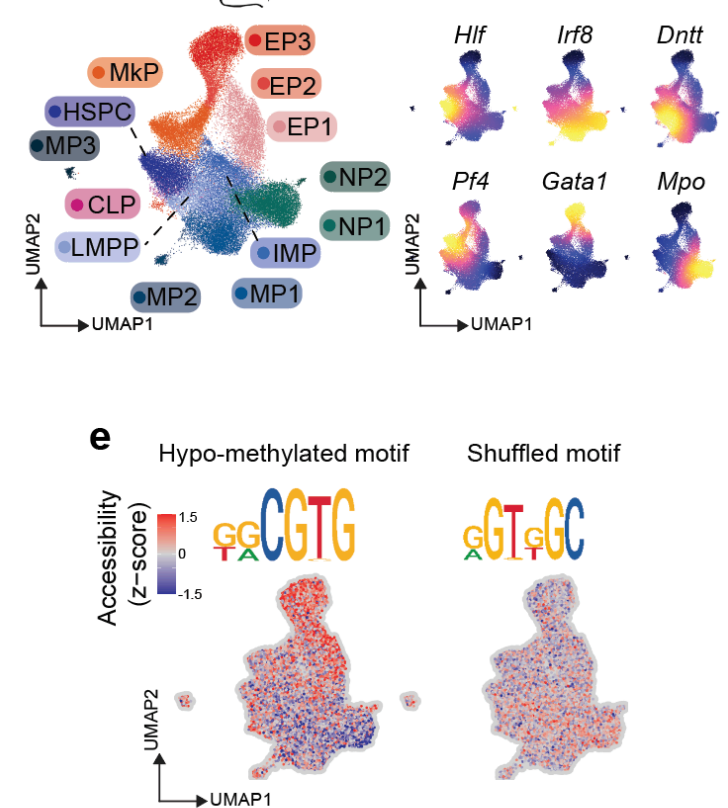
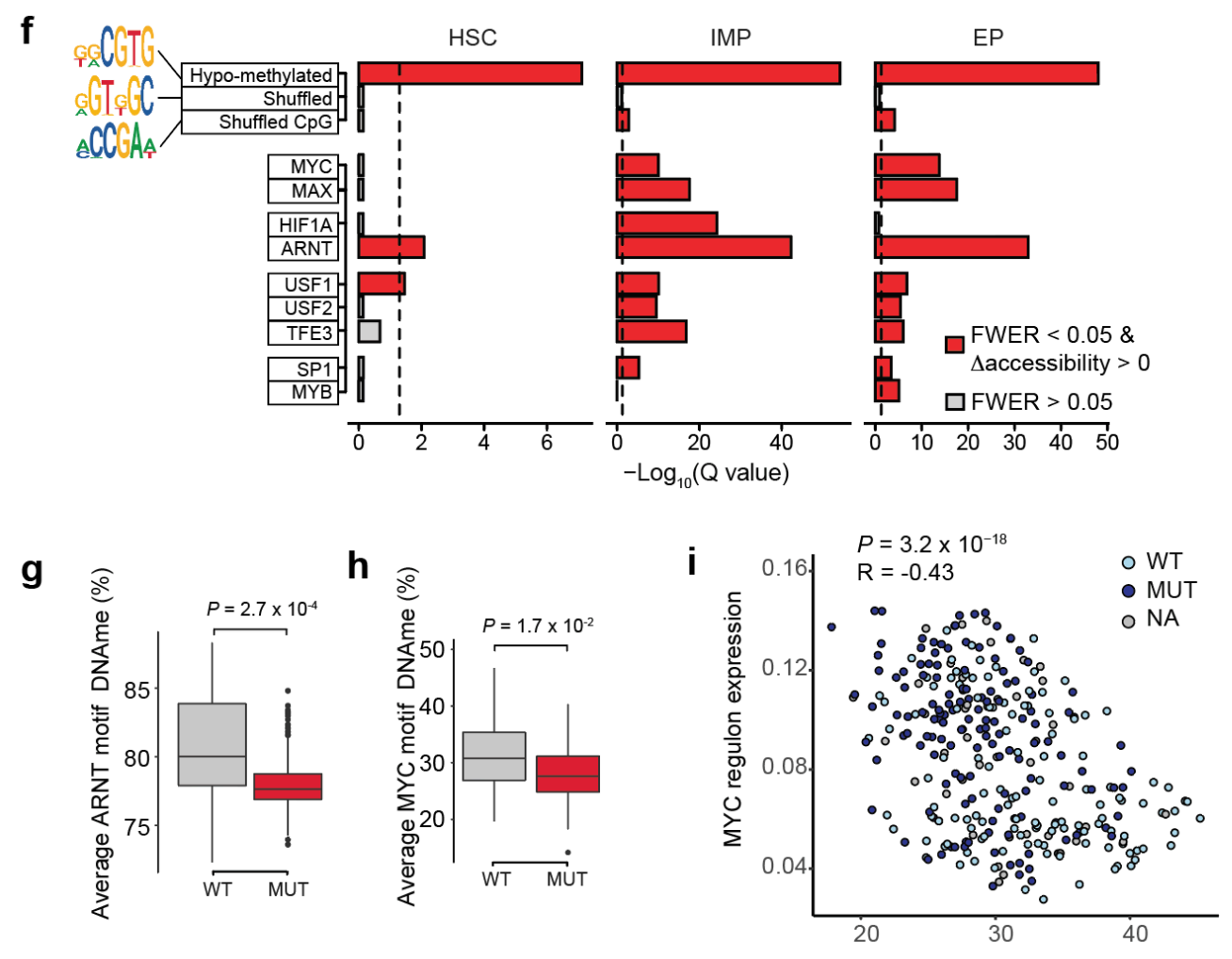

Average MYC Motif Methylation (\%) 
Figure 5. DNMT3A R882 displays flanking sequence specificity associated with MYC binding motif. a, Motif logo for the odds ratio of base frequency of the flanking positions $(\mathrm{N}-1, \mathrm{~N}-2, \mathrm{~N}+1, \mathrm{~N}+2)$ of CpG sites. Odds ratios were calculated based on the flanking regions of CpG sites hypomethylated or hypermethylated in DNMT3A R882 mutant compared with wildtype hematopoietic progenitors (online methods). b, Similarity score between the hypomethylated motif of DNMT3A R882 (Fig. 5a) and TF binding motifs in the HOCOMOCO v11 collection of human TF binding motifs. Relevant transcription factors with expression level in HSPCs $>0.5$ and motif similarity $>0.5$ are labeled. c, Frequencies of DNMT3A R882 hypomethylated motif within $30 \mathrm{~kb}$ of TSS of the differentially expressed genes between MUT and WT cells in HSPCs and EPs (identified in GoT data, Fig. 3a,c, see Extended Data Fig. 9d for other progenitor subsets, Extended Data Fig. 9e for $10 \mathrm{~kb}$ and $50 \mathrm{~kb}$ of TSS, and Extended Data Fig. 9f for data accounting for CpG content). P-values were calculated by Wilcoxon rank sum test. d, UMAP dimensionality reduction of murine wildtype ( $\mathrm{n}=3$ mice) and Dnmt3a R878H ( $\mathrm{n}=3$ mice) Lin', Kit ${ }^{+}$snATAC-seq data showing progenitor cluster annotation and representative progenitor gene marker accessibility ( $n=46,496$ cells). e, UMAP showing accessibility deviation as calculated with chromVar for hypomethylated motif (left) and shuffled motif (right, z-scores). f, Bonferroni FWER-adjusted P-values for accessibility changes between wildtype and Dnmt3a R878H cells by progenitor identities for hypomethylated motif and negative control shuffled motifs (with/without $\mathrm{CpG}$ ), as well as binding motifs of the TFs identified in Fig. 5b. g, Comparison of single cell average methylation of ARNT binding motifs (intersected with ARNT ChIPseq peaks, ENCODE hg38 Tf clusters) between wildtype and DNMT3A R882 mutant hematopoietic progenitor cells. P-values from likelihood ratio test of LMM with/without mutation status. h, Comparison of single cell average methylation of MYC binding motifs (intersected with MYC ChIP-seq peaks, ENCODE hg38 Tf clusters) between wildtype and DNMT3A R882 mutant hematopoietic progenitor cells. P-values from likelihood ratio test of LMM with/without mutation status. i, Relative expression per cell (AUC) of MYC downstream targets inferred using the SCENIC package (online methods) as a function of average MYC motif methylation. Correlation coefficient $\mathrm{R}$ calculated using Pearson's Correlation. P-value derived from GLM. HSPC, hematopoietic stem progenitor cells; MP, multipotent progenitors; IMP, immature myeloid progenitors; LMPP, lympho-myeloid primed progenitors; CLP, common lymphoid progenitor; EP, erythroid progenitor; MkP, megakaryocytic progenitor; NP, neutrophil progenitor.

consisted of mostly mutated cells with a high VAF (0.4), enabling a direct comparison to previously published healthy control CD34+ bone marrow cells ( $\mathrm{n}=39,082$ cells, Supplementary Table 9, online methods) ${ }^{141,142}$. We batch-corrected and integrated across the samples as previously described ${ }^{44}$ (Fig. 6a,b, Extended Data Fig. 11a-e). We first determined whether the bone marrow CH IMPs may display the lineage biases as previously observed in the CH01-CH04 samples. Consistent with those results, the IMPs from $\mathrm{CH} 05$ demonstrated skewing toward the ME versus GM state, compared to the control bone marrow CD34+ cells (Fig. 6c, Extended Data Fig. 12a-c). Next, we assessed the progenitorspecific differentially expressed genes identified in the CH01-CH04 samples and confirmed the expected increased or decreased expression for the differentially upregulated or downregulated genes in mutated cells, respectively, in $\mathrm{CH} 05$ progenitors, compared to control progenitors (data for HSPCs and EPs in Fig. 6d, Extended Data Fig. 12d,e, see other progenitors in Extended Data Fig. 12f). Furthermore, we observed an enrichment of the MYC/MAX target genes in the CH05 progenitors compared to the control progenitor cells (Fig. 6e), again most pronounced within the EPs. Intriguingly, the $\mathrm{CHO5}$ cells integrated evenly across progenitor subsets with control CD34+ cells except for a subcluster of EPs (EP2, Fig. 6a-b, Extended Data Fig. 12g). We suspected that the MYC/MAX target gene expression may be particularly impacted in this aberrant cluster and identified this to be the case (Fig. 6e, right). While the low genotyping efficiency limited our ability to make within cluster mutated versus wildtype comparisons in this sample, we were able to confirm across clusters the increased expression of differentially upregulated genes identified in more than one progenitor subset (Extended Data Fig. 12h-j, genes from Fig. 3b). Lastly, to test whether CD9 protein expression was impacted by the upregulation of the gene expression observed in the mutated HSPCs from CH01$\mathrm{CH} 04$, we incorporated protein expression in this sample through CITE-seq ${ }^{143}$. As CD9 expression has been linked with megakaryocytic differentiation priming59,60, we examined CD9 protein expression in the in the early $\mathrm{CD}^{4}{ }^{+}, \mathrm{CD} 38^{\text {low }}$ hematopoietic stem and progenitor cells along the megakaryocytic differentiation trajectory and observed an increased CD9 expression in mutated compared with wildtype cells (Extended Data Fig. 12k,l).

To test the chromatin accessibility of TF motifs (as a surrogate for TF activity) that bear high similarity to the DNMT3A R882 hypomethylated motif directly in this $\mathrm{CH}$ sample, we extended GoT to the $10 \mathrm{x}$ Multiome (ATAC+RNA) platform and applied it to sorted CD34+ nuclei (Fig. 6f, Extended Data Fig. 13a-c, n = 3,824 nuclei, note that the transcriptome data failed QC metrics and was not used downstream). As genotyping efficiency depends on mRNA abundance, the lower mRNA abundance in nuclei limited genotyping. We therefore again took advantage of the high VAF $(\sim 80 \%$ cells are mutant) and showed that across these cells, the accessibility of the hypomethylated motif - as well as those of MYC/MAX, HIF1A/ARNT, USF1/2/TFE3 - was increased compared to a shuffled motif and that of MYB, which may serve as an additional negative control (Fig. 
bioRxiv preprint doi: https://doi.org/10.1101/2022.01.14.476225; this version posted January 16,2022 . The copyright holder for this preprint (which was not certified by peer review) is the author/funder, who has granted bioRxiv a license to display the preprint in perpetuity. It is made available under aCC-BY-NC-ND 4.0 International license.
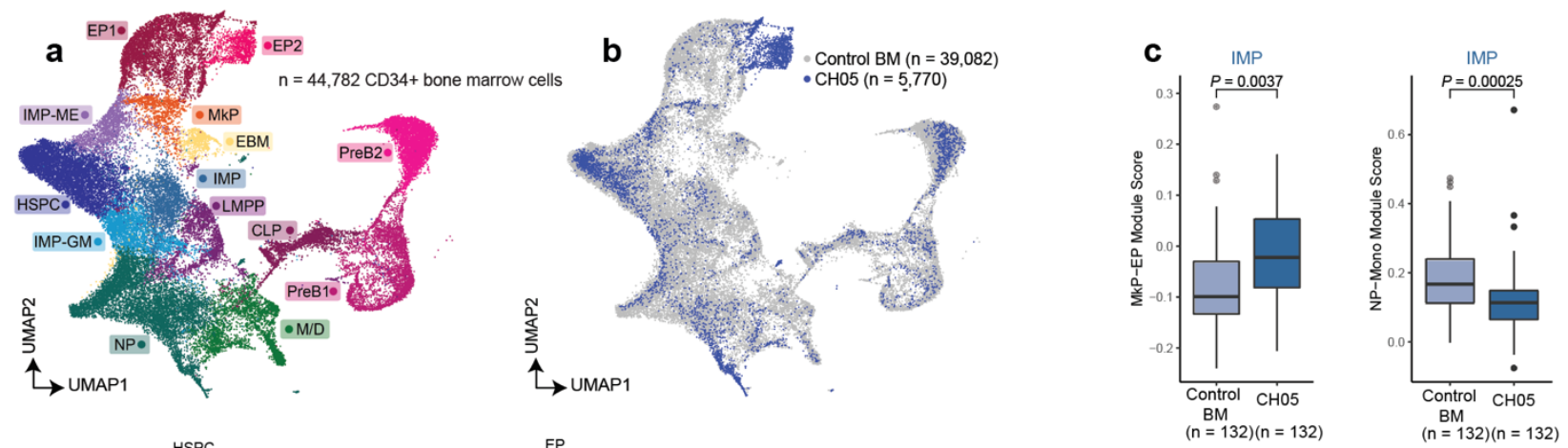

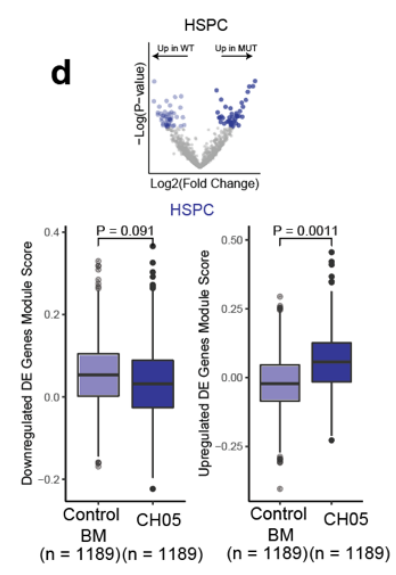

f

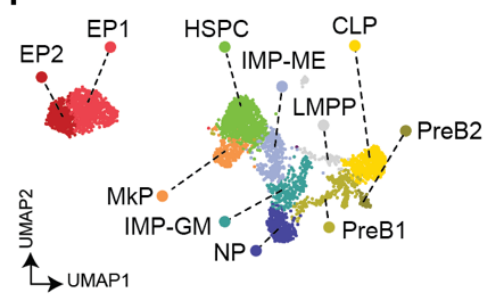

EP

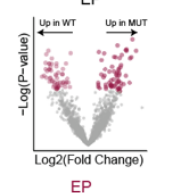

EP

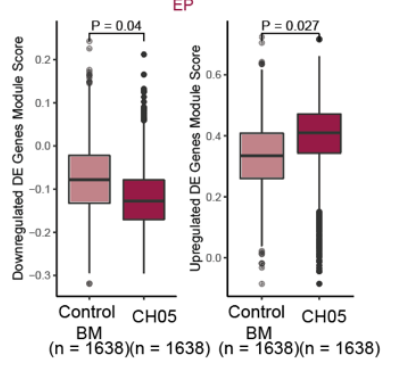

g

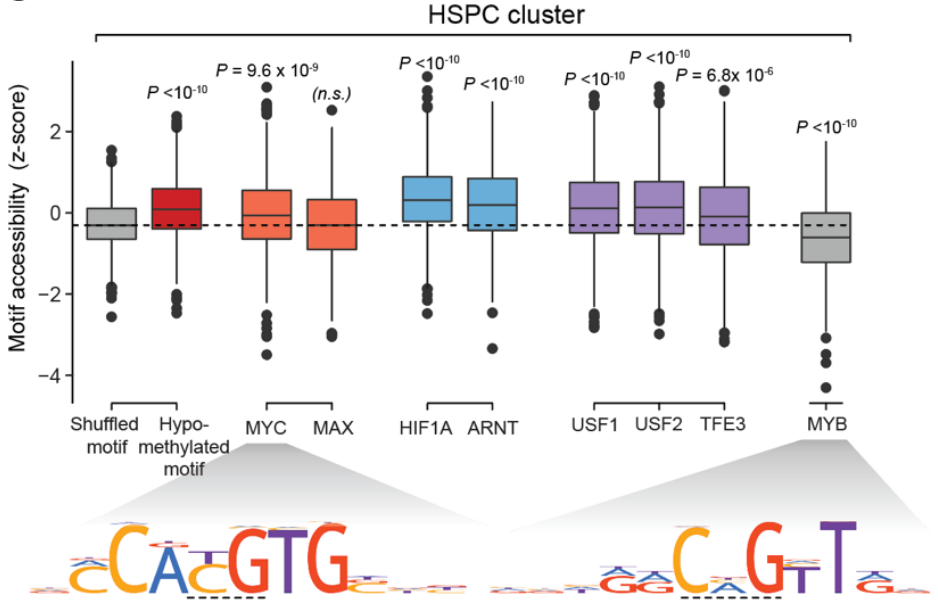

$\mathbf{e}$

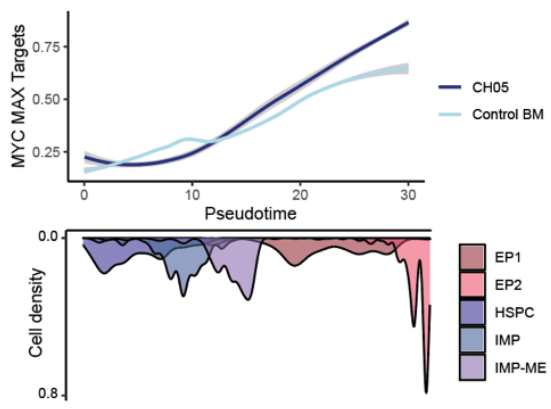

h

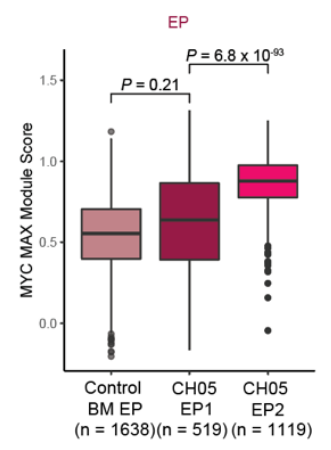

WT $(n=135)$ - MUT $(n=160)$

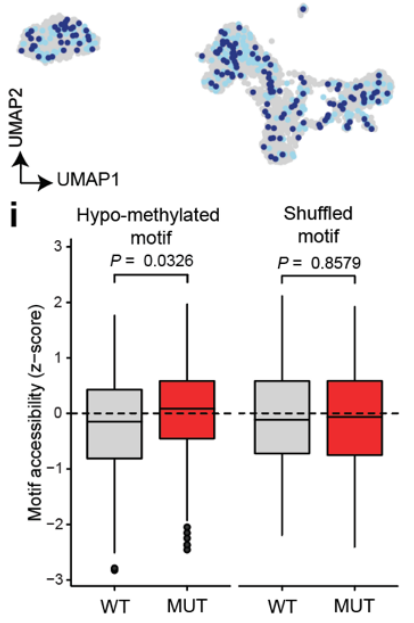

Figure 6. Bone marrow clonal hematopoiesis progenitor cells display megakaryocytic-erythroid differentiation bias, MYC target gene expression, and enhanced accessibility for the $\mathbf{R 8 8 2}$ hypomethylated motif. a, UMAP of CD34 ${ }^{+}$cells $(\mathrm{n}=$ 44,782 cells) for scRNA-seq data from a clonal hematopoiesis sample (CH05) and previously published five control bone marrow samples (BM01-05), labeled with cluster assignments. b, UMAP of CD34+ cells ( $n=44,782$ cells) labeled with $\mathrm{CH}(n=5,770)$ or control ( $n=39,082)$ status. c, Megakaryocytic-erythroid module scores in control versus CH IMPs (left, Supplementary Table 2) granulocytic-monocytic module scores in control versus CH IMPs (right, Supplementary Table 2). P-values were calculated from likelihood ratio test of LMM with/without $\mathrm{CH}$ status. d, Module scores for differentially down- or up-regulated genes in mutant DNMT3A HSPCs and EPs (identified in GoT data, Fig. 3a,c) in control versus CH HSPCs and EPs. e, Local regression of normalized expression levels as a function of pseudotime of MYC/MAX targets (differentially upregulated in Fig. 3c) for control and DNMT3A R882 CH cells. Shading denotes 95\% confidence interval. Histogram shows cell density of clusters included in the analysis, ordered by pseudotime. Boxplot shows comparison of module scores between control and $\mathrm{CH}$ cells within the two EP clusters. P-value calculated from likelihood ratio test of LMM with/without $\mathrm{CH}$ status. f, UMAP dimensionality reduction of CD34 ${ }^{+}$cells $(n=3,824$ cells) for snATAC-seq data from a clonal hematopoiesis sample (CH05) depicting the cell cluster assignment and cell type labels. g, Motif accessibility z-scores for shuffled, hypo-methylated motif and relevant transcription factors for the HSPC cluster ( $\mathrm{n}=788$ cells). P-values correspond to Wilcoxon rank sum test between accessibility of the shuffled motif and the indicated motif. $\mathbf{h}$, UMAP projection of genotype assignment for WT ( $\mathrm{n}=135 \mathrm{cells}$ ) and MUT ( $\mathrm{n}=160$ cells). i, Motif accessibility z-score comparison for either hypo-methylated or shuffled motifs between WT ( $\mathrm{n}=135$ cells), MUT ( $\mathrm{n}=160$ 
cells). P-values were calculated by Wilcoxon rank sum test. HSPC, Hematopoietic stem and progenitor cell; IMP- ME, immature myeloid progenitor with megakaryocytic/erythroid bias; IMP-GM, immature myeloid progenitor with granulocyte/monocyte bias; LMPP, Lymphoid-myeloid pluripotent progenitor; MkP, Megakaryocyte progenitor; NP, Neutrophil progenitor; CLP, Common lymphoid progenitor; Pre-B1/2, Pre-B cell progenitor; EP1/2, Erythroid progenitor.

6g). The accessibility of the hypomethylated motif increased with erythroid differentiation but not with lymphoid differentiation, consistent with the importance of these highlighted TFs in erythroid differentiation (Extended Data Fig. 13d,e). Finally, even within the limited number of genotyped cells, we observed that the accessibility of the hypomethylated motif was increased overall in the mutated cells compared to the wildtype-enriched population (Fig. 6h,i). In summary, these findings in a bone marrow DNMT3A-mutated $\mathrm{CH}$ sample, not complicated by exposure to G-CSF or prior chemotherapy, corroborated the findings in samples CH01-CH04, suggesting that the comparisons between mutated and wildtype cells within the same individuals are indeed robust to the potentially confounding extrinsic factors and are largely generalizable to steady-state DNMT3A R882-mutated $\mathrm{CH}$.

\section{Discussion}

We present an unbiased profiling of the downstream effects of somatic driver mutations in clonal mosaicism of normal human tissue, focusing on DNMT3A mutations in clonal hematopoiesis. Hitherto, extensive genetic profiling across normal tissues has been performed to document the striking mosaicism that result from pervasive age-related acquisition of somatic mutations ${ }^{1-}$ 5. For example, a landmark study of morphologically normal skin from the eyelids of four individuals identified $\sim 140$ mutations per square centimeter ${ }^{5}$. Importantly, while these studies have demonstrated that mutations in cancer drivers are particularly prevalent ${ }^{5}$, the downstream effects of cancer driver mutations that enable clonal outgrowths in normal human tissue are largely unknown.

Similarly, $\mathrm{CH}$ is a prevalent phenomenon in physiological hematopoietic aging fueled by driver mutations linked with myeloid neoplasms. However, the downstream consequences of these mutations in normal human hematopoietic progenitors are largely unknown. Previous studies leveraged rare germline mutations in small cohorts of patients to study the downstream perturbations of these mutations ${ }^{104,111}$. For example, by examining mature blood cells from an individual with Tatten-Brown-Rahman Syndrome (TBRS) due to a germline DNMT3A R882H mutation, with a sibling control104, the Ley group demonstrated focal hypomethylation, including of $\mathrm{CpG}$ islands, consistent with our findings. More recently, the Goodell group studied the effects of DNMT3A R771Q mutation by transforming primary cells into a lymphoblastoid cell line (LCL) from an early embryonal mosaic individual ${ }^{144}$, demonstrating significant overlap in hypomethylated regions in these DNMT3A R771Q LCLs and DNMT3A mutated AML.

Nonetheless, we previously lacked the ability to directly compare mutated and wildtype progenitors in human $\mathrm{CH}$ in their native context. Specifically, two obstacles challenge the study of $\mathrm{CH}$ mutation impact directly in primary patient samples. First, $\mathrm{CH}$ specimens with enriched human hematopoietic progenitors are scarce, as individuals with $\mathrm{CH}$ have no current clinical indication for a bone marrow biopsy. To circumvent this limitation, we pursued an alternative approach to profile $\mathrm{CH}$ mutated cells in stem cell graft products obtained from a cohort of MM patients in remission ${ }^{145}$ and identified one DNMT3A R882H CH bone marrow specimen without GCSF exposure or a potentially confounding cancer diagnosis to validate our findings. Second, mutated cells are admixed with wildtype in the hematopoietic progenitor pool and are morphologically and phenotypically indistinct. Thus, mutated cells cannot be isolated from wildtype cells for downstream analysis. We overcame this challenge by leveraging single-cell multi-omics that enabled us to profile the transcriptomes and epigenomes, together with the genotype information, of these single cells.

The application of the GoT approach ${ }^{38}$ enabled highresolution mapping of DNMT3A R882 mutated cells to the hematopoietic differentiation tree to reveal differentiation skewing, even before clinically observable changes in blood counts. We observed a myeloid over lymphoid bias, consistent with prior murine studies 51 , and the strong association of this genotype with myeloid versus lymphoid neoplasms. We further identified expansion of mutated IMPs and MEbiased IMPs. Enrichment of mutated cells in IMPs was linked with a specific increase in proliferation compared to wildtype cells. Notably, myeloid-bias has been linked with proinflammatory signaling64,146, and thus a proinflammatory state in mutated HSPCs (i.e. as evidenced by the overexpression of TNFRSF4, TYROBP, FCER1G) may also contribute to the enrichment of mutated cells in IMPs. Mutated IMPs further displayed a megakaryocytic-erythroid lineage bias, with enhanced transition probability of mutated IMPs to differentiate into IMP-MEs, consistent with our previous study in 
Dnmt3a KO mouse model ${ }^{54}$, as well as a Dnmt3a R878H model showing increased platelet counts ${ }^{64}$.

As DNMT3A R882-induced changes in DNAme are globally distributed across the genome, we sought to understand how stochastic DNAme changes can be translated into deterministic outputs, especially with respect to differentiation skews. We found that the DNMT3A R882 variants displayed a CpG sequence motif specificity, disfavoring CpGs with $\mathrm{T}$ at the $\mathrm{N}+1$ position, consistent with deep enzymology assays ${ }^{119}$. Notably, this hypomethylated $\mathrm{CpG}$ flanking motif bore high similarity to the binding motifs of key hematopoietic TFs, such as MYC/MAX, HIF1A/ARNT, USF1/2, providing a mechanistic model for enhanced MYC activity observed in our GoT data. This model was supported by mouse Dnmt3a R878H and, critically, human $\mathrm{CH}$ bone marrow data in which snATAC-seq of hematopoietic progenitors revealed enhanced accessibility of the hypomethylated motif and importantly of the MYC/MAX, HIF1A/ARNT, USF $1 / 2$ binding motifs. The accessibility changes associated with the hypomethylated motif were specifically pronounced in myeloid versus lymphoid progenitors, suggesting that these molecular consequences may play a role in differentiation biases. Furthermore, our single-cell multi-omics platform further enabled us to identify that cells with hypomethylation of MYC/MAX binding motifs showed increased expression of their transcriptional targets within the same cells, consistent with previous reports that demonstrated the negative impact on MYC activity imparted by the methylation of its binding motif121,122,139,140. These data revealed how modest, global, stochastically distributed DNAme changes can be translated into phenotypic skews. Through differences in the enrichment of $\mathrm{CpG}$ flanking sequence density of TF DNA binding motifs, subtle global DNAme changes affecting hundreds of binding sites can modulate TF output to result in reshaping of the differentiation landscape ${ }^{54}$.

We further identified preferential hypomethylation of PRC2 targets. While the relationship between PRC2mediated histone methylation and DNA methylation is not fully understood, DNA methylation may serve to "lock in" gene silencing with a mechanism with more robust mitotic inheritance ${ }^{147}$. PRC2 targets in stem cells include pluripotency/stemness genes ${ }^{148-150}$, and are enriched for bivalent H3K27me3/H3K4me3 marks 151,152 , suggesting that PRC2 results in "poising" rather than in complete silencing at those sites. In contrast, more differentiated cells reinforce gene silencing by increasing the length of H3K27me3 domains, or through complementary silencing mechanisms including DNA methylation 114-116. Thus, while PRC2 targets are broadly suppressed in stem cells, some leaky transcription may still occur, compared to PRC2 targets that have also underwent DNA methylation. This nuanced model posits that PRC2 targets DNA hypomethylation in DNMT3A mutated progenitors, may allow for their re-activation in response to stimuli, as another candidate mechanism for enhanced self-renewal through de-repression of stem cell programs. As activation of stem cell markers such as those repressed by the polycomb group proteins have been implicated in endowing cancer with stem-like properties ${ }^{153}$, our data points to poising of PRC2 targets as a potential mechanism for enhanced stem cell renewal upon malignant transformation. While PRC2 deficiency has been reported to lead to overexpression of stem cell maintainers such as $\mathrm{HoxC4}$ and inhibitors of differentiation such as Sox 7 and $I d 2$ in a murine model (Eed KO), as well as relative expansion of LT-HSC117, Eed KO cells also showed reduced competitive repopulating capacities with pro-apoptotic predisposition ${ }^{117}$. These data suggest that PRC2 target activation of self-renewal requires cooperation of an oncogenic TF such as MYC to counterbalance the proapoptotic effects and support clonal expansion in DNMT3A R882 cells. In support of this model, a recent work in mice demonstrated that while Ezh2 KO itself had little impact on hematopoiesis (likely due to redundant homologs), Ezh2 KO together with a compounding oncogenic driver (Nras G12D) promoted myeloid malignancy with activation of stemness genes ${ }^{154}$. Interestingly, Nras G12D alone promoted GM over ME bias, but in the double Ezh2 KO, Nras G12D mutated model, hematopoiesis was shifted toward ME over GM, suggesting that the PRC2 aberrations may indeed play a role in the observed ME bias (in addition to the better-established role of MYC in ME differentiation) ${ }^{154}$.

A potential limitation of our study of stem cell grafts is the exposure to G-CSF used in stem cell mobilization from patients with MM (of note, patients were not subject to other mobilization agents, such as CXCR4 antagonists or cyclophosphamide). Nonetheless, our analyses uniquely compared mutated and wildtype cells within the same sample, which were equally subjected to G-CSF. Indeed, our CH05 bone marrow aspirate sample from an individual with $\mathrm{CH}$ and no cancer diagnosis confirmed the major findings of the study, showing that comparing mutated versus wildtype cells from the same individuals is robust to the potential extrinsic confounders. For example, although G-CSF stimulates granulocytic differentiation and proliferation 155 , we were still able to capture the megakaryocytic-erythroid bias in the early mutated progenitors. Importantly, GCSF is especially effective in mobilizing quiescent murine HSCs, without inducing proliferation ${ }^{156}$. 
Interestingly, in the context of cell line models of DNMT3A R882, G-CSF induced a differentiation block in vitro in one study ${ }^{34}$ and GM-CSF masked the proliferative effects of the mutation in another ${ }^{157}$. Although these results were observed in cell lines, and thus the applicability to human $\mathrm{CH}$ is less clear, these data suggest that G-CSF may serve as a confounder. In this context, our validation of the major findings in a $\mathrm{CH}$ sample without exposure to G-CSF is of particular importance.

Another limitation results from the incomplete capture of the heterozygous allele in our GoT cDNA amplicon method due to low expression (median of 1 amplicon per genotyped cell, range 1-4 UMIs per cell). This is likely to result in misclassification of some mutated cells as wildtype cells. Nonetheless, as this is expected to diminish mutation-specific signals, the mutation-specific aberrations reported herein may likely have an even stronger effect size. Another limitation of the study is the sample size, due to the rarity of available samples. In this context, it is important to note that intensive profiling of a small number of samples (e.g. mutational profiling of normal eyelid samples from four individuals ${ }^{5}$ or epigenetic profiling of one TBRS patient with germline DNMT3A R882H mutation ${ }^{104}$ ) have shown that fundamental insights can be gained from these cases, directly in human samples. Our single-cell multi-omics profiling of thousands of progenitors, directly comparing mutated and wildtype cells within the same individuals, thus enabled us to highlight reproducible gene expression perturbations and epigenetic underpinnings, that were supported by evidence from published reports and murine data.

Altogether, we report the first direct examination of the molecular consequences of DNMT3A R882 mutations in primary $\mathrm{CD} 4^{+}$cells in human $\mathrm{CH}$. These studies allowed us to directly superimpose the differentiation topographies of mutated and wildtype hematopoietic progenitors, co-existing within the same individuals. We identified key epigenetic and transcriptional aberrations that reshape the differentiation topography and contribute to clonal expansion in the most nascent stage of neoplasia. These data also demonstrate the power of emerging single-cell multi-omics methods ${ }^{158-161}$ to pave the road towards defining how mutations drive normal tissue mosaicism in human somatic evolution.

\section{Acknowledgments}

The work was enabled by the Weill Cornell Epigenomics Core and Flow Cytometry Core. We thank Dr. Ari Melnick (Weill Cornell Medicine) for a critical review of the manuscript. A.S.N. is supported by the Burroughs
Wellcome Fund Career Award for Medical Scientists and National Institutes of Health Director's Early Independence Award (DP5 OD029619-01). N.D. is supported by a F30 Predoctoral Fellowship from the NHLBI of the National Institutes of Health (F30HL156496). N.D. and R.M.M. are supported by a Medical Scientist Training Program grant from the National Institute of General Medical Sciences of the National Institutes of Health under award number T32GM007739 to the Weill Cornell/Rockefeller/Sloan Kettering Tri-Institutional MD-PhD Program. F.I. is supported by the American Society of Hematology Fellow-to-Faculty Scholar Award. R.C. is supported by Lymphoma Research Foundation and Marie Skłodowska-Curie fellowships. D.A.L. is supported by the Burroughs Wellcome Fund Career Award for Medical Scientists, Valle Scholar Award, and the National Institutes of Health Director's New Innovator Award (DP2-CA239065). This work was also supported by the National Heart Lung and Blood Institute (R01HL145283) and the National Human Genome Research Institute, Center of Excellence in Genomic Science (RM1HG011014).

\section{Competing interests}

O.A.-W. has served as a consultant for H3B Biomedicine, Foundation Medicine Inc, Merck, Pfizer, and Janssen, and is on the Scientific Advisory Board of Envisagenics Inc and AIChemy; 0.A.-W. has received prior research funding from $\mathrm{H} 3 \mathrm{~B}$ Biomedicine and LOXO Oncology unrelated to the current manuscript. I.G. serves on the advisory board of Bristol Myers Squibb, Takeda, Janssen, Sanofi and GlaxoSmithKline. D.A.L. has served as a consultant for Abbvie and Illumina, and is on the Scientific Advisory Board of Mission Bio and C2i Genomics; D.A.L. has received prior research funding from BMS and Illumina unrelated to the current manuscript.

\section{METHODS}

\section{Patient samples}

The study was approved by the local ethics committee and by the Institutional Review Board (IRB) of Weill Cornell Medicine, University of Chicago and Dana Farber Cancer Institute conducted in accordance to the Declaration of Helsinki protocol. All patients provided informed consent. Cryopreserved G-CSF mobilized stem cell grafts (without additional mobilizing agents such as plerixafor or cyclophosphamide) from patients in remission for multiple myeloma, with documented DNMT3A R882 mutations were retrieved after interrogating a cohort of 136 patients with $\mathrm{CH}^{40}$. See Supplementary Table 1 for clinical information. 
Cryopreserved grafts were thawed and stained using standard procedures $\left(10 \mathrm{~min}, 4^{\circ} \mathrm{C}\right)$ with the surface antibody CD34-PE-Vio770 (clone AC136, lot\# 5180718070, dilution 1:50, Miltenyi Biotec) and DAPI (Sigma-Aldrich). Cells were then sorted for DAPInegative, CD34+ cells using BD Influx at the Weill Cornell Medicine flow cytometry core.

\section{Mouse Models}

All animals were housed at Memorial Sloan Kettering Cancer Center (MSKCC). All animal procedures were completed in accordance with the Guidelines for the Care and Use of Laboratory Animals and were approved by the Institutional Animal Care and Use Committees at MSKCC. The Dnmt3a R878H mouse model has been described previously51, and was crossed to the Tal1creERT2 transgenic model to allow for inducible control of the $\mathrm{R} 878 \mathrm{H}$ mutation within the hematopoietic system ${ }^{162}$. To induce recombination of the conditional alleles, age and gender-matched 10-16 week old Tal1creERT2 control mice and Dnmt3a R878H Tal1-creERT2 mice were treated with tamoxifen $(4 \mathrm{mg} / \mathrm{kg} /$ day; Cayman Chemical, Ann Arbor, Michigan) for 2 doses, separated 2 days apart. The mice were sacrificed 4-8 weeks after tamoxifen-induction. Primary mouse bone marrow (BM) cells were isolated into cold phosphatebuffered saline (PBS), without $\mathrm{Ca}^{2+}$ and $\mathrm{Mg}^{2+}$, and supplemented with $2 \%$ bovine serum albumin (BSA) to generate single cell suspensions. Red blood cells (RBCs) were removed using ammonium chloride-potassium bicarbonate (ACK) lysis buffer, resuspended in PBS/2\% BSA, and filtered through a $40 \mu \mathrm{m}$ cell strainer. Total nucleated cells were quantified by Vi-Cell XR cell counter (Beckman Coulter, Brea, CA) and used for downstream data production.

\section{Genotyping of Transcriptomes (GoT)}

Genotyping of Transcriptomes was performed as previously described ${ }^{38}$. The standard 10x Genomics Chromium 3' (v.3 chemistry) libraries were carried out according to manufacturer's recommendations for the generation of scRNA-seq libraries (Fig. 1a). At the cDNA amplification step, $1 \mu \mathrm{L}$ of $1 \mu \mathrm{M}$ spike-in primer $\left(5^{\prime}-\right.$ GAGGTCAAACTCCATAAAGCAGGGC- 3') was added to increase the yield of DNMT3A cDNA. After cDNA amplification and cleanup with SPRI beads, $25 \%$ of the cDNA underwent the standard $10 x$ protocol per manufacturer recommendations. The unused cDNA was stored and $10 \%$ was subsequently used for targeted genotyping. For locus-specific amplification (GoT), two serial PCRs were performed with nested reverse primers (5' -CTTATGGTGCACTGAAATGGAAAGGG - 3' and 5' CCTTGGCACCCGAGAATTCCAGGTTTCCCAGTCCACTATA CTGACG - 3') and the generic forward SI-PCR were used to amplify the site of interest from the cDNA template
(10 PCR cycles each). The second locus-specific reverse primer contains a partial Illumina TruSeq Small RNA read 2 handle and a locus-specific region to allow specific priming. The SI-PCR oligo (10x Genomics) anneals to the partial Illumina TruSeq read 1 sequence, preserving the cell barcode (CB) and unique molecule identifier (UMI). After these rounds of amplification and SPRI purification to remove unincorporated primers, a third PCR was performed with a generic forward PCR primer (P5_generic, 5' AATGATACGGCGACCACCGAGATCTACAC - 3') to retain the CB and UMI together with an RPI-x primer (Illumina) to complete the P7 end of the library and add a sample index (6 cycles). The targeted amplicon library was subsequently spiked into the remainder of the $10 \mathrm{x}$ library to be sequenced together on a NovaSeq (Illumina). The cycle settings were as follows: 28 cycles for read 1, 98 cycles for read 2, 8 cycles for i7 and 8 cycles for i5 sample index.

\section{0x scRNA-seq data processing, alignment, cell-type classification and clustering}

10x data were processed using Cell Ranger (v3.0.1) with default parameters. Reads were aligned to the human reference sequence hg19. The genomic region of interest for genotyping was examined to determine how many UMIs with the targeted sequence were present in the conventional 10x data. The Seurat package (v.3.1) was used to perform unbiased clustering of the CD34+ sorted cells from patient samples ${ }^{163}$. In brief, for individual datasets, cells with UMI $<200$ or UMI $>3$ median absolute deviations from the median UMI, or mitochondrial gene percentage $>20 \%$, were filtered. The data were log-normalized using a scale factor of 10,000. Before clustering, the individual datasets (CH01CH04) were integrated and underwent batch-correction within Seurat, which implements canonical correlation analysis and the principles of mutual nearest neighbor ${ }^{44}$. Recommended settings were used for the integration (30 canonical correlation vectors for canonical correlation analysis in the FindIntegrationAnchors function and 30 principal components for the anchor weighting procedure in IntegrateData function). Following integration, potential confounders (specifically, number of UMIs per cell, proportion of mitochondrial genes, and patient sex) were regressed out of the data before principal component analysis was performed using variable genes using recommended settings (i.e. top 2000 variable genes using variance stabilizing transformation) ${ }^{44}$. The first statistically significant 30 principal components were used as inputs to the UMAP algorithm for cluster visualization 164 . Clusters were manually assigned on the basis of differentially expressed genes using the FindAllMarkers 
function using default settings (using all genes that are detected in a minimum of $25 \%$ of cells in either of the two comparison sets as input, and log-transformed fold change of 0.25 as the threshold). We identified 20 clusters in the integrated data, which were annotated according to canonical lineage markers identified previously in single-cell RNA-seq data of normal hematopoietic progenitor cells ${ }^{53}$. These clusters were collapsed into 11 main progenitor subsets based on expression of levels of these canonical markers (Extended Data Fig. 3b,c). Pseudotime analysis was performed using the Monocle3 $\mathrm{R}$ package using recommended parameters (v.0.2.1, Extended Data Fig. 4d) 50 . In order to specify the initial cluster of the pseudotime trajectory, we identified the cluster with the highest expression level of the HSPC gene module (Fig. 1b, Supplementary Table 2). The Slingshot R package (v.1.6.1) was used to isolate the minimum spanning tree for the LMPP and CLP subset of cells (Fig. 2a) with default parameters.

\section{IronThrone GoT for processing targeted amplicon sequences and mutation calling}

Analysis of the GoT library was carried out as described previously ${ }^{38}$. Briefly, amplicon reads were assessed for presence of the primer sequence and the expected sequence between the primer and the mutation site. Reads were also assessed for matching to the cell barcode list of the $10 \mathrm{x}$ dataset. A mismatch of $20 \%$ was allowed for all sequence matching steps. Only UMIs with at least 2 or more supporting reads were retained for final genotyping assignments. A few key improvements to our IronThrone pipeline (v.2.1) are detailed below.

First, parallelization was implemented to increase runtime efficiency for larger sequencing libraries 165 . The amplicon library of paired reads was shuffled and subsetted into smaller groups of reads (default 125,000 reads/group). Then, the original IronThrone algorithm was run on each one of these groups. This step has been parallelized using both GNU Parallel tools for local interactive operation, as well as options for Slurmmanaged high-performance compute clusters. Output tables from these runs are finally concatenated by cell barcode.

Second, we improved the UMI counting of the amplicon reads by removing 'pseudo'-UMIs introduced by PCR and sequencing errors (that would result in a false increase in the number of UMIs). Based on previously published work $^{42}$, we implemented a network-based UMI collapsing algorithm to aggregate amplicon reads that likely originated from the same UMI in the original 10x library. Briefly, pairwise Levenshtein distances were calculated between all UMIs paired within a single cell barcode, and "matches" between UMIs were identified as
UMI pairs with a Levenshtein distance below a predetermined threshold (default $=\operatorname{ceiling}(0.1 * \mathrm{UMI}$ length), or 2 bases for a 12 base UMI). The UMI with the greatest number of matched UMIs was determined to be the initial UMI. The number of supporting reads for these UMI groups was summed together and attributed to that initial UMI with the most matches. This process was then repeated for the UMI with the next highest number of matches until no additional collapsing was possible. This improved pipeline was applied to the previouslydescribed species mixing experiment ${ }^{38}$, demonstrating a significant improvement in the removal of aberrant genotyping UMIs (see Results, Extended Data Fig. 1e).

Following UMI collapse, genotype assignment of individual UMIs was conducted as described previously with majority rule of supporting reads for wildtype or mutant status. Rare UMIs with an equal number of mutant and wildtype reads were removed as ambiguous. Additionally, to remove reads that result from PCR recombination ${ }^{38}$, UMIs in the amplicon library that match UMIs of non-DNMT3A genes in the gene expression library were discarded. Of note, the latter likely PCR-recombination events were associated with lower number of read per UMI compared with UMIs in the amplicon library that matched DNMT3A UMI in the gene expression library (Extended Data Fig. 1f). We leveraged this observation, and retained UMIs without a corresponding associated gene in the gene expression library, so long as their read count was above the $80^{\text {th }}$ percentile of read counts for non-DNMT3A genes. Finally, single cells were assigned mutant or wildtype genotype status as follows: cells with one or more mutant UMIs were assigned as mutant cells, and cells with 0 mutant UMIs and at least one wildtype UMI were assigned as wildtype. While the genotyping information is derived from transcribed molecules and may be affected by the capture of transcripts from wildtype versus mutant alleles of heterozygous mutations, the frequency of mutant cells as determined by GoT using all cells that harbor at least one UMI yielded values that were similar to that determined by bulk DNA exon sequencing (Extended Data Fig. 1c).

\section{Mutant cell frequency analysis}

To exclude the possibility that variable DNMT3A expression may impact the ability to detect mutant alleles and thereby impact mutated cell frequency in distinct progenitor subsets, we down-sampled all cells to a single amplicon UMI prior to mutation calling for calculating mutant cell frequencies (Fig. 2a,b). An equal number of cells from each sample CH01-CH04 ( $\mathrm{n}=83$ cells for LMPP + CLP (Fig. 2a) and $n=978$ cells for analysis of all cell types (Fig. 2b)), were subsampled randomly for the integrated data. Genotyping amplicon 
UMIs were downsampled (x100 iterations) to one per cell and mutant cell frequency was determined for each cluster for either the integrated dataset or individual samples. This frequency was then divided by the total mutant cell frequency across all progenitor subsets for each of the iterations. Linear mixed effects analysis was performed using the lme4 package (v.1.2-1). Progenitor identity was defined as the fixed effect, and for random effects, we used intercepts for individual patients (subjects) and iterative downsampling. P-values were obtained by likelihood ratio tests of the full model with the fixed effect against the model without the fixed effect ${ }^{166}$.

\section{RNA velocity}

RNA velocity was calculated using scVelo (v0.2.2) ${ }^{57}$. For generating the loom file, the Python (v3.7) version of Velocyto (v0.17) ${ }^{56}$ was ran using the velocyto run command. The cell barcode and bam files were obtained using Cell Ranger. In addition to the cell barcode and bam files, a GTF file corresponding to the reference used for alignment (hg19; Ensembl 187) was supplied. Repetitive regions were masked using a GTF file downloaded from UCSC selecting for repetitive regions in GRCh37 (hg19). QC was assessed by the percent of unspliced reads per sample, requiring a minimum of $25 \%$ total unspliced reads. If duplicated gene names were present in the spliced and unspliced tables the counts were summed to leave only unique genes. Next, gene velocity for each patient and genotype was estimated separately using scVelo (v0.2.2). In order to avoid a potential confounder of unequal number of cells for each genotype, random sampling of the same number of mutant and wildtype cells to the minimum number in either group was performed for each patient sample for downstream analysis. Gene selection for RNA velocity estimation was performed requiring a minimum of 20 counts. After log-normalization by cell depth, the top 2,000 genes with the highest dispersion were selected for downstream calculations. Next, first and second order moments were computed among nearest neighbors in principal component space, using the pp.moments function with parameters n_pcs $=30$ and n_neighbors $=30$. RNA velocity was estimated using the dynamical model option of the tl.velocity function. The cell-to-cell transition probability matrices were retrieved for either wild type or mutant cells. For a given cell, we averaged the probabilities of transitioning to transcriptional states within a cluster of interest. This resulted in a mean probability of transition for the cell of interest to a given cluster. Statistical significance of the mean single cell differentiation probabilities between genotypes was estimated by linear mixed models. Sample was added as the random effect and genotype as the fixed effect. P-values were obtained by likelihood ratio tests of the full model with the fixed effect against the model without the fixed effect. To further compare wildtype to mutant probabilities for a given transition, we calculated the median of the distribution of singlecell mean transition probabilities toward other cell clusters, and calculated the mutant-to-wildtype odds ratio of the median probabilities.

\section{Gene module scoring, differential expression and gene set enrichment analysis}

For examining gene and gene module expression (see Supplementary Table 2), the function AddModuleScore was used to calculate the relative expression of the genes for each cell within the Seurat package (e.g. Fig. 2c; MkP-EP module score (union of the MkP and EP module genes in Supplementary Table 2) was calculated using the AddModuleScore function) ${ }^{44}$. Briefly, control gene module expressions were calculated and subtracted from the average gene module expression of interest, as previously described ${ }^{55}$. All analyzed genes were classified based on average expression into 24 bins, and for each gene in the module, 100 control genes are randomly selected from the same expression bin as the gene of interest ${ }^{55}$. For statistical analysis, genotype status was entered as the fixed effect and subjects as random effects in a linear mixed model. P-values were obtained by likelihood ratio tests of the full model with the fixed effect against the model without the fixed effect.

Differential expression analysis comparing wildtype and mutant cells was conducted using a within-sample permutation test for each progenitor cell subtype. Briefly, to ensure equal representation from each patient, the numbers of mutated and wildtype cells from each patient were downsampled to the same number, respectively. Observed log2 fold change values were calculated with original genotyping assignments (MUT versus WT) for the tested genes. The tested genes included the top 2,500 most variable genes which were filtered for those expressed in at least $5 \%$ of either group (mutated versus wildtype), for each progenitor subtype. Ribosomal and mitochondrial genes were excluded. Next, over 100,000 iterations, WT and MUT labels were shuffled within each patient, and fold change values were re-calculated to create a background distribution. P-values were calculated per gene as a percent of permutations whose absolute fold change values were more extreme than the absolute value of the observed fold change (Supplementary Table 3). As an orthogonal approach, we also performed differential expression analysis comparing wildtype and mutant cells via the linear mixed model framework. For each gene, genotype status was entered as the fixed effect and subjects as 
random effects. P-values were obtained by likelihood ratio tests of the full model with the fixed effect against the model without the fixed effect (Supplementary Table 3).

Hypergeometric test for gene set enrichment analysis of the integrated differentially expressed genes (P-value < $0.05, \log 2$ (fold change) $>0.25$ ) was performed using the Cluster Profile package (v. 0.1.9) ${ }^{167}$. FDR multiple hypothesis testing correction was performed. MSigDB C2: Chemical and genetic perturbations (CGP) sources were included in the analyses (Supplementary Table 4).

\section{Copy number variation analysis}

The InferCNV package (v.1.4.0) ${ }^{43}$ was used to analyze the single cell dataset for any duplications or deletions of entire chromosomes or large chromosome fragments. Briefly, by comparing expression levels of genes annotated by chromosomal position (using the CONICSmat package, v0.0.0.1168) to a set of reference cells (in this case, a one-versus-rest comparison of cells by patient of origin), a heatmap of relative expression can be generated and used to identify regions with significantly increased or decreased expression. We removed the few genes for which alternative positions have been reported ( $<2 \%$ of genes). We downsampled our dataset to 978 genotyped cells from each patient (the minimum number of genotyped cells from any given individual patient). We then ran the InferCNV workflow with recommended parameters, using the i6 6-state Hidden Markov model (Extended Data Fig. 2a). As a positive control, we specifically analyzed relative expression of Y-chromosome genes to ensure sexdifferences between patients were appropriately reflected in our data (Extended Data Fig. 2b).

\section{Hypomethylated motif enrichment analysis in differentially expressed genes}

The HOMER (v4.9) scanMotifGenomeWide function was used to search for occurrences of the DNMT3A R882 hypomethylated motif and a control motif containing a CpG. For each gene in the scRNA-seq dataset, TSS coordinates were identified and a .bed file was created with intervals of $\pm 10 \mathrm{~kb}, 30 \mathrm{~kb}$ or $50 \mathrm{~kb}$ surrounding each TSS. These two sets of coordinates were intersected using bedtools (v2.30.0), and the number of hypomethylated motif or control motif sites were counted per gene. Differentially expressed genes were classified as upregulated $(\mathrm{P}<0.05, \log 2$ (fold change) $>$ 0.25 ) or downregulated ( $<<0.05, \log 2$ (fold change) $<-$ 0.25 ), and counts of hypomethylated motif sites were compared, with P-values obtained by Wilcoxon rank sum test. To ensure that the results were not driven simply by the presence of a $\mathrm{CpG}$, we also determined the ratio of the counts of the hypomethylated motif to that of the control shuffled motif with $\mathrm{CpG}$ per gene.

\section{Joint multiplexed single-cell methylome and single- cell RNA-seq library construction}

DNA methylation data was processed produced as previously described by Gaiti et al. ${ }^{39}$ Briefly, genomic DNA (gDNA) and mRNA were separated as follows. A modified oligo-dT primer (5'-biotin-triethyleneglycolAAGCAGTGGTATCAACGCAGAGTACT30VN-3', where V is either $A, C$ or $G$, and $N$ is any base; IDT) was conjugated to streptavidin-coupled magnetic beads (Dynabeads, Life Technologies) according to the manufacturer's instructions. To capture polyadenylated mRNA, we added the conjugated beads $(10 \mu \mathrm{l})$ directly to the cell lysate and incubated them for $20 \mathrm{~min}$ at room temperature with mixing to prevent the beads from settling. The mRNA was then collected to the side of the well using a magnet, and the supernatant, containing the gDNA, was transferred to a fresh plate. Single-cell complementary DNA was amplified from the tubes containing the captured mRNA according to a variation of the Smart-Seq2 protocol 107 using molecular crowding to increase sensitivity ${ }^{169}$. After amplification and purification using $0.8 \mathrm{X}$ SPRI beads, $0.5 \mathrm{ng}$ cDNA was used for Nextera Tagmentation and library construction. At the cDNA amplification step, the following primers were spiked-in $(0.5 \mu \mathrm{M}$ final) to specifically increase capture of the locus around DNMT3A R882 mutation (Fw: 5 TCGTCGGCAGCGTCAGATGTGTATAAGAGACAGGGTTTC CCAGTCCACTATACTGACG-3' ; Rv: 5'TCGTCGGCAGCGTCAGATGTGTATAAGAGACAGATGACC GGCCCAGCAGTCTC -3'). The same primers were used to specifically amplify the target locus separately in a portion of the cDNA. Library quality and quantity were assessed using Agilent Bioanalyzer 2100 and Qubit, respectively. Libraries were then sequenced with paired-end, 50-base reads, using a NovaSeq sequencer (Illumina).

Genomic DNA present in the pooled supernatant and wash buffer from the mRNA isolation step was concentrated on $0.8 X$ SPRI beads and eluted directly into the reaction mixtures for single digest or Msp1 + HaeIII (Fermentas) for double restriction enzyme digest reaction $\left(10 \mu \mathrm{L}\right.$ final reaction) for $90 \mathrm{~min}$ at $37^{\circ} \mathrm{C}$. Heatinactivation was performed for $10 \mathrm{~min}$ at $70^{\circ} \mathrm{C}$. Digested DNA was filled-in and A-tailed at the $3^{\prime}$ sticky ends in 8.5 $\mu \mathrm{L}$ final volume of $1 \mathrm{X}$ CutSmart with 2.5 units of Klenow fragment (Exo-, Fermentas). Reaction was supplemented with $1 \mathrm{mM}$ dATP and $0.1 \mathrm{mM} \mathrm{dCTP}$ and $0.1 \mathrm{mM}$ dGTP (NEB) and performed as follows in a thermocycler: $30^{\circ} \mathrm{C}$ for $25 \mathrm{~min}, 37^{\circ} \mathrm{C}$ for $25 \mathrm{~min}$, and $70^{\circ} \mathrm{C}$ for $10 \mathrm{~min}$ (heat-inactivation). Custom barcoded 
methylated adaptors $(0.1 \mu \mathrm{M})$ were then ligated overnight at $16^{\circ} \mathrm{C}$ with the dA-tailed DNA fragments in the presence of 800 units of T4 DNA ligase (NEB) and 1 mM ATP (Roche) in a final volume of $11.5 \mu \mathrm{L}$ of $1 \mathrm{X}$ CutSmart buffer. T4 DNA ligase heat-inactivation was performed at $70^{\circ} \mathrm{C}$ for $15 \mathrm{~min}$ the next day. Genomic DNA from 24 individual cells were pooled together according to their barcodes, giving 4 pools of 24 cells for a 96-well plate. Pooled genomic DNA was cleaned-up and concentrated using 1.8X SPRI beads (Agencourt AMPure $\mathrm{XP}$, Beckman Coulter). Each pool was then converted using an enzyme-based conversion to increase the recovery of single cell gDNA compared to standard bisulfite conversion (NEBNext Enzymatic Methyl-seq, New England Biolabs) ${ }^{102}$. Standard bisulfite conversion was implemented for double restriction enzyme digest reactions, as previously described ${ }^{107}$. Converted DNA was then amplified using primers containing Illumina i7 and 15 index. Following Illumina pooling guidelines, a different i7 and i5 index was used for every 24-cell pool, allowing multiplexing of several samples for sequencing on Illumina NovaSeq6000. Library enrichment was done using KAPA HiFi Uracil+ master mix (Kapa Biosystems) and the following PCR condition was used: $98^{\circ} \mathrm{C}$ for 45 secs; 6 cycles of: $98^{\circ} \mathrm{C}$ for 20 secs, $58^{\circ} \mathrm{C}$ for 30 secs, $72^{\circ} \mathrm{C}$ for $1 \mathrm{~min}$; followed by 12 cycles of: $98^{\circ} \mathrm{C}$ for $20 \mathrm{secs}, 65^{\circ} \mathrm{C}$ for $30 \mathrm{secs}, 72^{\circ} \mathrm{C}$ for $1 \mathrm{~min}$. PCR was terminated by an incubation at $72^{\circ} \mathrm{C}$ for $5 \mathrm{~min}$. Enriched libraries were cleaned-up and concentrated using 1.3X SPRI beads. DNA fragments between $200 \mathrm{bp}$ and $1 \mathrm{~Kb}$ were sizeselected and recovered after resolving on an E-Gel EX Precast Agarose Gels (Thermo Fisher Scientific). Library molarity concentration calculation was obtained by measuring concentration of double stranded DNA (Qubit) and quantifying the average library size (bp) using an Agilent Bioanalyzer. Every 24-cell pool was mixed with the other pools in an equimolar ratio. Negative controls (empty wells with no cells) were used to control for non-specific amplification of the libraries.

\section{Multimodal single cell methylome and RNA sequencing data processing}

Methylation analysis pipeline. DNA methylation data was processed as previously described ${ }^{39}$. Pools of 24 cells were demultiplexed based on a supplied list of cell barcodes. Adapter sequences were trimmed by the first 3 bp on each 3' end of R1 and R2. Bismark (v0.14.5) was used to create bisulfite-converted genomes of GRCh38 (hg38 Ensembl version 93). Reads were mapped using Bismark with Bowtie (v2.2.8) and default alignment parameters. BAM files were then used to run Bismark methylation extractor ignoring $6 \mathrm{bp}$ from the end of R1 and $5 \mathrm{bp}$ from R2. This was done to remove technical variability introduced at the ends of the reads during end repair with unmethylated nucleotides. These settings were determined from the M-bias reports, which contain the methylation proportion at each read position. Bismark methylation extractor (-bedgraph comprehensive) was used to determine the methylation state of each individual CpG. Cells with $>99 \%$ conversion efficiency as determined by Bismark were retained for downstream analysis. Reads mapping to ChrY and the mitochondrial genome were removed from the resulting .cov files. For all downstream analysis, the methylation status of CpGs per cell was binarized. CpGs with 10-90\% methylation values were removed $(<2 \%$ of total CpGs) and those with values $<10 \%$ were encoded as 0 , while those with values $>90 \%$ are encoded as 1 . On average, $209,519 \pm 15,200( \pm$ SEM) unique CpGs per cell were covered in the DNA methylome.

RNA analysis pipeline. scRNA-seq data was aligned using STAR (v2.5.2a). Default parameters were used, other than twopassMode Basic. Reads were aligned to GRCh38 (hg38 Ensembl version 93). Gene counts were determined using featureCounts from Subread (v1.5.2) using default parameters. Ensembl gene IDs were converted to hgnc symbols using the R package biomaRt (v2.40.5). In cases where there were duplicated gene symbols the counts were summed. Seurat (v3.1.1) was then used to analyze gene expression data. Cells were filtered for mitochondrial reads of less than $25 \%$ and a minimum of 200 detected genes. Genes were filtered for coverage across at least three cells. The mean $( \pm$ standard deviation) number of detected genes was 5,763 \pm 2075 genes/cell (range 3,117 $\pm 678-8,715 \pm 1,449$ genes/cell across the plates). The mean number of reads was $511,840 \pm 315,941 \mathrm{reads} /$ cell (range 170,383 \pm $63,951-779,771 \pm 361,887$ reads/cell across the plates). Normalization and variable feature detection were performed for each batch (i.e. plate). Batch correction and integration was performed via the Seurat integration pipeline ${ }^{44}$ using recommended parameters for SelectIntegrationFeatures, FindIntegrationAnchors, and IntegrateData. Dimensionality reduction was performed by principal component analysis using the RunPCA function, and the first 12 principal components were retained for downstream analysis. For visualization, UMAP164 was performed using the RunUMAP function. Cell type assignment was performed as described for the 10x Genomics scRNA-seq data.

Genotyping. To process genotyping data, genotyping FASTQs were aligned the same manner as RNA library FASTQs. Pysam (v0.8.2.1) was used to select reads overlapping the target allele by using the pileup function. Reads were filtered by a minimum read mapping quality (MAPQ) of 40 and a minimum base quality (Phred score) of 20. Each remaining read was classified as either 
mutant or wildtype based on the nucleotide detected at the mutation site based on bulk sequencing data ${ }^{40}$. Cells were classified as mutant if there were at least two mutant reads, and wildtype if there were at least three wildtype reads (increased stringency given mutation heterozygosity) and no mutant reads. For genotyping libraries with increased sequencing depth $(7,712 \pm 319$ versus $20 \pm 2.75$ reads; mean \pm SEM), the base quality thresholds were increased to 40 . For genotype classification, a bootstrapping approach was implemented by randomly sampling 50 reads for 100 iterations. For each iteration, a mutant fraction cutoff of 0.10 was applied. The final genotyping call was performed in cells with above $80 \%$ bootstrap support.

\section{Average Single Cell Methylation}

We compared single cell methylation at selected genomic regions (i.e. enhancers, $\mathrm{CpG}$ islands, ChIP-seq peaks) between mutant and wildtype cells from each patient. To achieve this, we first filtered for CpG sites with coverage in at least three cells in each patient, in order to reduce inter-patient variability. The genomic region of interest was then intersected with the CpG sites using the R package GenomicRanges (v1.36.1). Finally, the average methylation for a given region across the covered CpG sites was calculated for each cell. Statistical significance between genotypes was estimated by linear mixed models. Sample was added as the random effect and genotype as the fixed effect. P-values were obtained by likelihood ratio tests of the full model with the fixed effect against the model without the fixed effect. Due to potential differences between single versus double digest data, we display single digest datasets as representatives (unless otherwise indicated for analysis that specifically relies on the enhanced coverage of double digest).

\section{Single-cell differentially methylated region (DMR) analysis to identify preferential hypomethylation}

For each cell, Bismark methylation extractor output files (containing information on methylation state of each individual $\mathrm{CpG}$ ) were intersected with the genomic regions of interest (e.g., promoters) using BEDTools (v2.27.1). A generalized linear model (GLM) was then built to predict the DNAme for a given genomic region between genotypes, accounting for global methylation changes. For each cell, the global DNAme value was defined as the average DNAme across all genomic regions investigated. The model used was as follows:

$$
\mathrm{GLM}=\mathrm{m}_{\mathrm{i}, \mathrm{j}} \sim \mathrm{g}_{\mathrm{i}}+\mathrm{t}_{\mathrm{i}}
$$

Where $m_{i, j}$ represents the average DNAme of the genomic region $\mathrm{j}$ (e.g., promoter of FOXA2) for cell $\mathrm{i}$; $\mathrm{g}_{\mathrm{i}}$ represents the genotype of cell $i$ and $t_{i}$ represents the average methylation for all CpGs detected in cell i. Only genomic regions with sufficient DNAme information ( $>5$ CpGs per region for promoters and $>50$ CpGs for ChIPSeq peaks) in at least 15 cells per group (mutated or wildtype) were used in the analysis. To test the impact of genotype on DNAme for a given genomic region (e.g. promoter of FOXA2), P-values were derived from the GLM (calculated from the t-statistic computed by dividing the genotype $(\mathrm{g})$ regression coefficient by the residual standard error, Supplementary Table 5). To calculate the percentage methylation difference in mutant cells for a given genomic region of interest, the average across mutant and wildtype cells was taken within plate to control for batch effects. Next, the DNAme difference between mutant and wildtype was computed within plate and a weighted average of the difference was calculated, using the number of cells from each plate as weights. In order to be consistent across genes, promoters were defined as $1 \mathrm{~kb}$ upstream and $1 \mathrm{~kb}$ downstream of transcription start sites (hg38 RefSeqGene) ${ }^{109}$. ChIP-seq peaks were obtained from ENCODE (hg38 Tfbs clustered) ${ }^{109}$. When directly examining the methylation status of SUZ12 and EZH2 targets, we intersected the ENCODE ChIP-seq peaks with bivalent peaks (H3K27me3, H3K4me3) from human $\mathrm{CD}^{4} 4^{+}$hematopoietic progenitor cells ${ }^{110}$.

Gene set enrichment analysis. To define the pathways enriched at hypo- or hypermethylated TSS, genes were ranked based on methylation difference, and differentially hypomethylated genes $(\mathrm{P}<0.05)$ were selected as inquiry for pathway analysis. We note that gene set enrichment analysis of RRBS data may be confounded by the fact that the use of restriction enzymes enriches for $\mathrm{CpG}$ rich genomic regions as well as $\mathrm{CpG}$ rich promoters. Thus, pathway enrichment was performed via a pre-ranked gene set enrichment approach (and thus including only genes covered in our data) using the msigdbr (v7.2.1) and fgsea (v1.12.0) R packages, with the MSigDB C2 CGP collection of curated gene sets.

\section{DNMT3A R882 motif analysis}

CpG flanking motif analysis. To identify the sequences surrounding hypo or hypermethylated $\mathrm{CpG}$ sites in wildtype versus DNMT3A mutant hematopoietic progenitors, we first performed differentially methylated regions (DMR) analysis in $\mathrm{CpG}$ islands as described above in the "Single-cell differentially methylated region (DMR) analysis" section. CpGs within hypo or hypermethylated regions $(\mathrm{P}<0.05)$ were selected, and the surrounding \pm 6 bp sequences were extracted using bedtools (v2.25.0). The frequency of each base pair at each position relative to the CpG site was calculated, and statistical significance was assessed by Fisher exact test. Odds ratio logo was generated by 
calculating the frequency for each base at each position for either hypomethylated or hypermethylated CpG sites. To identify differentially enriched bases surrounding the CpG site, we applied increasingly stringent thresholds on the absolute methylation difference required between wildtype and mutated cells to consider the sites, and estimated the odds ratio of base frequency of hypo- over hyper-methylated sites at a given position relative to the $\mathrm{CpG}$ site. Next, we calculated the correlation between the methylation difference required and the odds ratio of base frequency. We define bases differentially enriched or depleted in hypo- versus hyper-methylated based on the correlation significance $(\mathrm{P}<0.05)$. For $\mathrm{CpG}$ sites with greater than absolute methylation difference of 0.5 , the odds ratios were computed and used as input to generate the logo using the ggseqlogo (v0.1) package. To identify transcription factors with the motif pattern of interest, we used the HOCOMOCO v11 human motif position weight matrix (PWM) collection in HOMER format with $P<0.001$. For each of the PWMs, we selected the position containing the highest $\mathrm{CpG}$ probability and calculated the similarity score of the flanking -2 and +2 positions relative to the $\mathrm{CpG}$ site against the hypo-methylated flanking sequences, based on the correlation of the base frequencies along each of the motifs.

\section{Average methylation at MYC motifs and modeling regulon expression}

The MYC and ARNT motif PWM was downloaded from the HOCOMOCO (v11) human TF database and used as input to HOMER (v4.9). The scanMotifGenomeWide function was used to search for occurrences of motifs throughout the genome. The R package GenomicRanges (v1.36.1) was used to intersect CpG sites with motifs and respective ChIP-seq peaks (ENCODE database) ${ }^{109}$. Methylation per cell was then averaged across the covered CpG sites. Positively regulated downstream MYC targets were determined using pySCENIC (v0.10.0). Counts were converted to transcripts per million (TPM) and genes in the count matrices were filtered for those in the cisTarget database (all available hg38 files were used). The hgnc (v9) motif file from the cisTarget database was used to generate a list of input motifs. Regulons were determined from each patient sample separately with default parameters as described ${ }^{170}$. To analyze expression of the regulons, per-cell AUC scoring was done using the aucell function. The relationship between MYC motif methylation and regulon expression was modeled with a generalized linear model (GLM) using a Gamma distribution with the following model:

$$
\mathrm{GLM}=\mathrm{r}_{\mathrm{i}} \sim \mathrm{m}_{\mathrm{i}}
$$

Where $r_{i}$ represents the AUC score to MYC downstream targets for cell $i ; m_{i}$ represents the DNAme of MYC motifs for cell i. Due to batch effects between methylome sequencing methods, only samples that were prepared using the enzymatic method were included. Rare outliers were excluded that had a Cook's distance greater than $2 *$ mean Cook's distance. To test the impact of MYC motif methylation on regulon expression, the Pvalue was derived from GLM output (calculated from the t-statistic computed by dividing the MYC motif methylation $(\mathrm{m})$ regression coefficient by the residual standard error).

\section{AML PRC2 target methylation analysis}

Methylated base call files of DNMT3A-mutated AML samples were downloaded from Glass et al.105 PRC2 targets were obtained from the union of EZH2 and SUZ12 ChIP-seq peaks (see "single-cell differential methylation analysis"), as approximately $50 \%$ of SUZ12 ChIP-seq peaks overlapped with EZH2 peaks. PRC2 targets were further intersected with promoters using the GenomicRanges (v1.38.0) findOverlaps function, requiring at least $30 \mathrm{bp}$ to be overlapping. We note that PRC2 targets are known to have a higher CpG content 171,172 , potentially biasing the result given the higher coverage of RRBS of high CpG content promoters. We therefore also compared PRC2 target methylation only with high $\mathrm{CpG}$ content promoters as annotated by Saxonov et al. 173 and $\pm 1 \mathrm{~KB}$ surrounding the TSS. For each sample 270,000 CpG sites were randomly sampled from either promoters overlapping with PRC2 peaks, or non-overlapping promoters as a control. The number of randomly sampled $\mathrm{CpG}$ sites was selected based on the minimum coverage among replicates. The ratio of methylation between DNMT3A mutant and wildtype AML (Fig. 4h), required to pair each mutated AML with a wildtype sample. As this pairing is arbitrary (i.e., samples are not explicitly matched), to safeguard against a non-representative pairing, we permutated all possible pairing and P-values were obtained by Wilcoxon rank sum test. The example shown represents the median Pvalue among the permutations. Methylated base call files of DNMT3A-mutated and wildtype AML samples were downloaded from TCGA ${ }^{118}$. Overlap of PRC2 ChIP-seq peaks and promoter regions was carried out as described above. The average methylation at high $\mathrm{CpG}$ promoters that overlap with PRC2 peaks and high CpG promoters that do not overlap with PRC2 peaks was calculated per sample and compared between DNMT3A R882 mutant and DNMT3A wildtype AML (Wilcoxon rank sum test).

\section{Single nucleus ATAC-sequencing of Dnmt3a R878 and wildtype HSPCs}

Hematopoietic progenitors (Lin-1-, c-Kit ${ }^{+}$) were sorted from wildtype ( $\mathrm{n}=3$ mice) or Dnmt3a R878H ( $=3$ mice) via c-Kit enrichment as directed by the 
manufacturer (CD117 Microbeads, clone 3C1, Miltenyi, Auburn, CA; LS Columns (Cat. No. \#130-042-401), Miltenyi) followed by FACS (Lin-1 BV421 (Cat. No. \#133311), Biolegend, San Diego, CA; CD117 APC (clone 2B8, Invitrogen, Waltham, MA). Nuclei isolation was performed as suggested by the manufacturer $(10 \mathrm{x}$ Genomics, Pleasanton, CA). Briefly, single cell suspensions were centrifuged at $300 \mathrm{rcf}$ for 5 minutes and cell pellets were resuspended in $100 \mu \mathrm{l}$ of lysis buffer (Tris-HCl pH 7.4, 10mM; $\mathrm{NaCl} 10 \mathrm{mM} ; \mathrm{MgCl}_{2} 3 \mathrm{mM}$; Tween-20 0.1\%; Nonidet P40 substitute (Sigma-Aldrich, St. Louis, MO) 0.1\%; Digitonin 0.01\%; BSA 1\%; DTT 1 $\mathrm{mM}$; RNase inhibitor $1 \mathrm{U} / \mu \mathrm{L}$ (Sigma-Aldrich, St. Louis, MO)) and kept on ice for 3 minutes. Then, $1 \mathrm{ml}$ of wash buffer (Tris- $\mathrm{HCl} \mathrm{pH} \mathrm{7.4,10mM;} \mathrm{NaCl} 10 \mathrm{mM} ; \mathrm{MgCl}_{2} 3 \mathrm{mM}$; BSA 1\%; Tween-20 0.1\%; DTT $1 \mathrm{mM}$; Sigma Protector RNase inhibitor $1 \mathrm{U} / \mu \mathrm{L}$ ) was added. The isolated nuclei were centrifuged for $5 \mathrm{~min}$ at $500 \mathrm{rcf}$, and pellets were resuspended in Diluted Nuclei Buffer (10x Genomics Nuclei Buffer 1X; DTT $1 \mathrm{mM}$; Sigma Protector RNase inhibitor $1 \mathrm{U} / \mu \mathrm{L}$ ). Nuclei concentration was determined by hemocytometer and processed as indicated by the manufacturer (10x Genomics User Guide: Chromium Next GEM Single Cell Multiome ATAC + Gene Expression, CG000338). Single nucleus ATAC and Gene Expression (GEX) libraries were constructed in parallel and assessed for quality control metrics using Agilent Bioanalyzer 2100 and Qubit respectively. ATAC libraries were sequenced to a depth of 25,000 read pairs per nucleus (paired-end, dual indexing: Read 1N 50 cycles, i7 Index 8 cycles, i5 Index 24 cycles, Read 2N 49 cycles) and GEX libraries were sequenced to a depth of 20,000 read pairs per nucleus (paired-end, dual indexing: 28 cycles for Read 1, 10 cycles for i7 Index, 10 cycles for i5 Index, 90 cycles for Read 2).

\section{Single nucleus ATAC-sequencing data processing}

Pre-processing was performed using 10x Genomics Cell Ranger ARC (v1.0.1). Reads were de-multiplexed using the cellranger-arc mkfastq function. Single cell feature counts for each sample were then generated using the cellranger-arc count function. The gene expression information for these libraries exhibited exceedingly low UMI and genes per cell consistent with lower quality RNA in single-cell nuclei Multiome data; as such, we moved forward utilizing only the ATAC data for analysis. ATAC data was processed using the ArchR package (v1.0.1) ${ }^{174}$ using the atac_fragments.tsv.gz file generated by the cellranger-arc count function as input. Arrow files were created using a minimum TSS enrichment score of 5 and a minimum number of unique nuclear fragments of 1,000. Doublet scores were calculated using the addDoubletScores function with $\mathrm{k}=10$, knnMethod $=$ "umap" and LSImethod $=1$. Doublets were removed using the filterDoublets function with default parameters. Dimensionality reduction was performed through iterative semantic index (LSI) using the cell by genomic window (500 bp) matrix as input, using the addIterativeLSI function with the following parameters: iterations $=3$, resolution $=0.2$, sampleCells $=1,000$, var.features $=25,000$ and dimsToUse $=1: 30$. Cell clusters were identified using the addClusters function using the iterative latent semantic index (LSI) dimensions as input, with method = "Seurat", resolution $=0.8$. For visualization, UMAP dimensionality reduction was performed using the LSI dimensions as input, using the addUMAP function with: $\mathrm{nNeighbors}=30$, minDist $=$ 0.5 and metric $=$ "cosine". Cell identities were assigned based on gene accessibility scores of known marker genes. Custom motif accessibility deviations were calculated as follows: position weight matrices in HOMER format $(P<0.001)$ were downloaded from the HOCOMOCO v11 mouse database. Motif occurrences were identified using the scanGenomeWide function of the HOMER package. To include only high confidence motif sites, we applied a minimum odds ratio score threshold of 6 . We next created custom peakAnnotations using ArchR and performed ChromaVar analysis using the addDeviationsMatrix function with default parameters.

\section{CH05 sample processing and analysis}

\section{Single cell RNA-seq processing and downstream analysis}

CH05 bone marrow underwent sorting, scRNAsequencing and genotyping with GoT as described above for samples CH01-04, with the exception of the addition of the CITE-seq integration. Briefly, the Total-seqA antibodies (Biolegend: CD38, CD9, CD49f, CD45RA, CD41, CD36, CD69, CD42, CD14, CD71, CD45RB, CD45R0, CD37, CD7, CD279, CD47, CD90, CD99, CD84, CD274, FLT3, CD79B, CD45, CD81) were used according to manufacturer's recommendations. The $\mathrm{CD} 34^{+}$sorted cells were incubated with the antibodies for 30 minutes and underwent washes $3 \mathrm{X}$. 10x data were processed using Cell Ranger (v3.0.1) with default parameters. Reads were aligned to the human reference sequence hg19. Control bone marrow samples (BM01-05) were identified from previously published reports 141,142 with raw count matrices available for download. The Seurat package (v.3.1) was used to perform integration and unbiased clustering of the $\mathrm{CD}_{34}{ }^{+}$sorted cells from patient samples as described previously with the following notable exceptions ${ }^{163}$. The publicly available archived count matrices for samples BM04 and BM05 had the following QC filtering: the mitochondrial and ribosomal genes were removed, and only cells with > 400 unique genes and between 1,000 and 10,000 UMIs 
were kept. Consequently, these two patients were not filtered with the aforementioned criteria. $\mathrm{CH} 05$ and BM01-03 were filtered identically as samples CH01-04, following which mitochondrial and ribosomal genes were removed from the gene expression matrix. All samples were then normalized and integrated as described previously, with the exception of proportion of mitochondrial genes no longer being regressed out as a potential confounder. We identified 26 clusters in the integrated data, which were annotated as above using lineage markers previously identified for normal hematopoietic progenitors ${ }^{53,175}$.

Following cell-type assignment, we down-sampled the count matrices using the downsampleBatches function from the scuttle package (v1.0.4) to ensure that the average per-cell geometric mean of raw counts was consistent across all 6 patient samples ${ }^{176}$.

Module scores were calculated as described above. The performance of the CITE-seq antibodies was assessed based on expected expression patterns across the progenitor subsets.

\section{Single nucleus ATAC-seq and downstream analysis}

snATAC-seq data for $\mathrm{CH} 05$ was generated as described above using the Multiome platform (10x Genomics) and GoT performed as described above using the cDNA generated from the Multiome workflow. The gene expression information for these libraries exhibited very low UMI and genes per cell consistent with lower quality RNA in single-cell nuclei Multiome data; as such, we moved forward utilizing only the ATAC data for analysis. For the analysis, fragment files were generated by processing the fastq files using cell-ranger-ARC (v.1.0.0). Downstream analysis was performed using the ArchR (v1.0.1) pipeline ${ }^{174}$. Based on the distribution of total fragments and TSS enrichment per cell, empty droplets were filtered out by requiring a minimum of 3,000 fragments per cell and a TSS enrichment score of 7.5. Potential doublets were detected using the addDoubletScores function, using KNN on the UMAP dimensionality reduction with $\mathrm{k}=10$. Cell barcodes with high enrichment for doublet scores were removed using the filterDoublets function with default parameters. Next, we performed dimensionality reduction through iterative latent semantic indexing (LSI) using the top 25,000 variable features. Cell clustering was performed using the addClusters function, with the following parameters: reduceDims = "IterativeLSI"; method = "Seurat"; resolution $=1$. For visualization, further dimensionality reduction was performed by applying UMAP to the iterative LSI space using the addUMAP function with the following parameters: nNeighbors = 30; minDist $=0.5 ;$ metric $=$ "cosine". Cell type identification was performed by manually inspecting the genes showing up-regulated gene accessibility scores (FDR $<0.01$ and $\log _{2}$ (fold change) $>1.25$ ) for each of the defined clusters (Extended Data Fig. 13c). Motif occurrences were defined using the position weight matrices (PWMs) obtained from the Hocomoco (v.11.0) motif database or our custom PWMs for hypomethylated and shuffled motifs using HOMER (v4.9), requiring a minimum enrichment score above 6 . Transcription factor, hypo-methylated and shuffled motif accessibility was calculated using ChromVAR 177 within the ArchR (v1.0.1) pipeline ${ }^{174}$. Supervised pseudotime trajectories for either erythroid or lymphoid fates were defined within the ArchR (v1.0.1) pipeline ${ }^{174}$ applying the addTrajectory function. 
bioRxiv preprint doi: https://doi.org/10.1101/2022.01.14.476225; this version posted January 16,2022 . The copyright holder for this preprint (which was not certified by peer review) is the author/funder, who has granted bioRxiv a license to display the preprint in perpetuity. It is made available under aCC-BY-NC-ND 4.0 International license.

\section{REFERENCES}

1. Martincorena, I. et al. Somatic mutant clones colonize the human esophagus with age. Science 362, 911-917 (2018).

2. Yizhak, K. et al. RNA sequence analysis reveals macroscopic somatic clonal expansion across normal tissues. Science 364(2019).

3. Yokoyama, A. et al. Age-related remodelling of oesophageal epithelia by mutated cancer drivers. Nature 565, 312-317 (2019).

4. Yoshida, K. et al. Tobacco smoking and somatic mutations in human bronchial epithelium. Nature (2020).

5. Martincorena, I. et al. High burden and pervasive positive selection of somatic mutations in normal human skin. Science 348, 880-886 (2015).

6. Mustjoki, S. \& Young, N.S. Somatic Mutations in "Benign" Disease. $N$ Engl J Med 384, 2039-2052 (2021).

7. Shlush, L.I. et al. Identification of pre-leukaemic haematopoietic stem cells in acute leukaemia. Nature 506, 328-333 (2014).

8. Jaiswal, S. et al. Age-related clonal hematopoiesis associated with adverse outcomes. $N$ Engl J Med 371, 2488-98 (2014).

9. Genovese, G. et al. Clonal hematopoiesis and blood-cancer risk inferred from blood DNA sequence. New England Journal of Medicine 371, 2477-2487 (2014).

10. Xie, M. et al. Age-related mutations associated with clonal hematopoietic expansion and malignancies. Nature Medicine 20, 1472-1478 (2014).

11. Steensma, D.P. et al. Clonal hematopoiesis of indeterminate potential and its distinction from myelodysplastic syndromes. Vol. 126 9-16 (American Society of Hematology, 2015).

12. Young, A.L., Challen, G.A., Birmann, B.M. \& Druley, T.E. Clonal haematopoiesis harbouring AML-associated mutations is ubiquitous in healthy adults. Nature Communications 7(2016).

13. Zink, F. et al. Clonal hematopoiesis, with and without candidate driver mutations, is common in the elderly. Blood 130, 742-752 (2017).

14. Champion, K.M., Gilbert, J.G.R., Asimakopoulos, F.A., Hinshelwood, S. \& Green, A.R. Clonal haemopoiesis in normal elderly women: Implications for the myeloproliferative disorders and myelodysplastic syndromes. British Journal of Haematology $\mathbf{9 7}$, 920-926 (1997).

15. SanMiguel, J.M. et al. Cell-Extrinsic Stressors from the Aging Bone Marrow (BM) Microenvironment Promote Dnmt3a-Mutant Clonal Hematopoiesis. Blood 134, 5-5 (2019).

16. Terao, C. et al. Chromosomal alterations among age-related haematopoietic clones in Japan. Nature, 1-6 (2020).

17. Watson, C.J. et al. The evolutionary dynamics and fitness landscape of clonal hematopoiesis. Science 367, 1449-1454 (2020).

18. Teixeira, V.H. et al. Deciphering the genomic, epigenomic, and transcriptomic landscapes of pre-invasive lung cancer lesions. Nat Med 25, 517-525 (2019).

19. Desai, P. et al. Somatic mutations precede acute myeloid leukemia years before diagnosis. Nat Med 24, 1015-1023 (2018).

20. Papaemmanuil, E. et al. Clinical and biological implications of driver mutations in myelodysplastic syndromes. Blood 122, 36163627 (2013).

21. Abelson, S. et al. Prediction of acute myeloid leukaemia risk in healthy individuals. Nature 559, 400-404 (2018).

22. Reddy, A. et al. Genetic and Functional Drivers of Diffuse Large B Cell Lymphoma. Cell 171, 481-494.e15 (2017).

23. Coombs, C.C. et al. Therapy-Related Clonal Hematopoiesis in Patients with Non-hematologic Cancers Is Common and Associated with Adverse Clinical Outcomes. Cell Stem Cell 21, 374-382 e4 (2017).

24. Fuster, J.J. et al. Clonal hematopoiesis associated with TET2 deficiency accelerates atherosclerosis development in mice. Science 355, 842-847 (2017).

25. Jaiswal, S. \& Ebert, B.L. Clonal hematopoiesis in human aging and disease. Vol. 366 (American Association for the Advancement of Science, 2019).
26. Jaiswal, S. \& Libby, P. Clonal haematopoiesis: connecting ageing and inflammation in cardiovascular disease. Nature Reviews Cardiology (2019).

27. Gibson, C.J. et al. Donor-engrafted CHIP is common among stem cell transplant recipients with unexplained cytopenias. Vol. 130 91-94 (American Society of Hematology, 2017).

28. Boettcher, S. et al. Clonal hematopoiesis in donors and long-term survivors of related allogeneic hematopoietic stem cell transplantation. Blood 135, 1548-1559 (2020).

29. Frick, M. et al. Role of Donor Clonal Hematopoiesis in Allogeneic Hematopoietic Stem-Cell Transplantation. J Clin Oncol 37, 375-385 (2019).

30. Buscarlet, M. et al. DNMT3A and TET2 dominate clonal hematopoiesis and demonstrate benign phenotypes and different genetic predispositions. Blood 130, 753-762 (2017).

31. Young, A.L., Spencer Tong, R., Birmann, B.M. \& Druley, T.E. Clonal hematopoiesis and risk of acute myeloid leukemia. Haematologica 104, 2410-2417 (2019).

32. Jeong, M. et al. Loss of Dnmt3a Immortalizes Hematopoietic Stem Cells In Vivo. Cell Reports 23, 1-10 (2018).

33. Ostrander, E.L. et al. Divergent Effects of Dnmt3a and Tet2 Mutations on Hematopoietic Progenitor Cell Fitness. Stem Cell Reports 14, 551-560 (2020).

34. Koya, J. et al. DNMT3A R882 mutants interact with polycomb proteins to block haematopoietic stem and leukaemic cell differentiation. Nat Commun 7, 10924 (2016).

35. Kim, S.J. et al. A DNMT3A mutation common in AML exhibits dominant-negative effects in murine ES cells. Blood 122, 40864089 (2013).

36. Russler-Germain, D.A. et al. The R882H DNMT3A Mutation Associated with AML Dominantly Inhibits Wild-Type DNMT3A by Blocking Its Ability to Form Active Tetramers. Cancer Cell 25, 442454 (2014).

37. Miles, L.A. et al. Single-cell mutation analysis of clonal evolution in myeloid malignancies. Nature 587, 477-482 (2020).

38. Nam, A.S. et al. Somatic mutations and cell identity linked by Genotyping of Transcriptomes. Nature 571, 355-360 (2019).

39. Gaiti, F. et al. Epigenetic evolution and lineage histories of chronic lymphocytic leukaemia. Nature 569, 576-580 (2019).

40. Mouhieddine, T.H. et al. Clonal hematopoiesis is associated with adverse outcomes in multiple myeloma patients undergoing transplant. Nat Commun 11, 2996 (2020).

41. Bick, A.G. et al. Inherited causes of clonal haematopoiesis in 97,691 whole genomes. Nature (2020).

42. Smith, T., Heger, A. \& Sudbery, I. UMI-tools: modeling sequencing errors in Unique Molecular Identifiers to improve quantification accuracy. Genome Research 27, 491-499 (2017).

43. Tickle, T., Tirosh, I., Georgescu, C., Brown, M. \& Haas, B. inferCNV of the Trinity CTAT Project. Klarman Cell Observatory, Broad Institute of MIT and Harvard (2019).

44. Stuart, T. et al. Comprehensive Integration of Single-Cell Data. Cell 177, 1888-1902 e21 (2019).

45. Pellin, D. et al. A comprehensive single cell transcriptional landscape of human hematopoietic progenitors. Nature Communications 10, 2395-2395 (2019).

46. Challen, G.A. et al. Dnmt3a and Dnmt3b have overlapping and distinct functions in hematopoietic stem cells. Cell Stem Cell 15, 350-364 (2014).

47. Challen, G.A. et al. Dnmt3a is essential for hematopoietic stem cell differentiation. Nat Genet 44, 23-31 (2011).

48. Cao, J. et al. The single-cell transcriptional landscape of mammalian organogenesis. Nature 566, 496-502 (2019).

49. Qiu, X. et al. Reversed graph embedding resolves complex singlecell trajectories. Nature Methods 14, 979-982 (2017).

50. Trapnell, C. et al. The dynamics and regulators of cell fate decisions are revealed by pseudotemporal ordering of single cells. Nature Biotechnology 32, 381-386 (2014).

51. Guryanova, O.A. et al. DNMT3A mutations promote anthracycline resistance in acute myeloid leukemia via impaired nucleosome remodeling. Nat Med 22, 1488-1495 (2016). 
bioRxiv preprint doi: https://doi.org/10.1101/2022.01.14.476225; this version posted January 16, 2022. The copyright holder for this preprint (which was not certified by peer review) is the author/funder, who has granted bioRxiv a license to display the preprint in perpetuity. It is made available under aCC-BY-NC-ND 4.0 International license.

52. Arends, C.M. et al. Hematopoietic lineage distribution and evolutionary dynamics of clonal hematopoiesis. Leukemia 32, 1908-1919 (2018).

53. Velten, L. et al. Human haematopoietic stem cell lineage commitment is a continuous process. Nat Cell Biol 19, 271-281 (2017).

54. Izzo, F. et al. DNA methylation disruption reshapes the hematopoietic differentiation landscape. Nature genetics, 1-10 (2020).

55. Tirosh, I. et al. Dissecting the multicellular ecosystem of metastatic melanoma by single-cell RNA-seq. Science 352, 189-96 (2016).

56. La Manno, G. et al. RNA velocity of single cells. Nature 560, 494-498 (2018).

57. Bergen, V., Lange, M., Peidli, S., Wolf, F.A. \& Theis, F.J. Generalizing RNA velocity to transient cell states through dynamical modeling. Nat Biotechnol 38, 1408-1414 (2020).

58. Iwasaki, H. \& Akashi, K. Hematopoietic developmental pathways: on cellular basis. Oncogene 26, 6687-6696 (2007).

59. Clay, D. et al. CD9 and megakaryocyte differentiation. Blood 97, 1982-1989 (2001).

60. Noetzli, L.J., French, S.L. \& Machlus, K.R. New insights into the differentiation of megakaryocytes from hematopoietic progenitors. Vol. 39 1288-1300 (Lippincott Williams and Wilkins, 2019).

61. Rubinstein, E., Billard, M., Plaisance, S., Prenant, M. \& Boucheix, C. Molecular cloning of the mouse equivalent of CD9 antigen. Thrombosis Research 71, 377-383 (1993).

62. Slupsky, J.R. et al. The Platelet Antigens CD9, CD42 and Integrin alphaIIbbetaIIIa Can be Topographically Associated and Transduce Functionally Similar Signals. European Journal of Biochemistry 244, 168-175 (1997).

63. Worthington, R.E., Carroll, R.C. \& Boucheix, C. Platelet activation by CD9 monoclonal antibodies is mediated by the $\mathrm{F}<\mathrm{sub}>\mathrm{C} \gamma</$ sub $>$ II receptor. British Journal of Haematology 74, 216-222 (1990).

64. Dai, Y.J. et al. Conditional knockin of Dnmt3a R878H initiates acute myeloid leukemia with mTOR pathway involvement. Proceedings of the National Academy of Sciences of the United States of America 114, 5237-5242 (2017).

65. Thol, F. et al. Incidence and prognostic influence of DNMT3A mutations in acute myeloid leukemia. Journal of Clinical Oncology 29, 2889-2896 (2011).

66. Xu, J. et al. DNMT3A Arg882 mutation drives chronic myelomonocytic leukemia through disturbing gene expression/DNA methylation in hematopoietic cells. Proceedings of the National Academy of Sciences of the United States of America 111, 2620-2625 (2014).

67. Saito, Y. et al. Identification of therapeutic targets for quiescent, chemotherapy-resistant human leukemia stem cells. Sci Transl Med 2, 17ra9 (2010).

68. Zhang, G., Bogdanova, N., Gao, T. \& Sheikh, K.A. Elimination of activating $\mathrm{F} c \gamma$ receptors in spontaneous autoimmune peripheral polyneuropathy model protects from neuropathic disease. Plos One 14, e0220250 (2019).

69. Tsuboi, N., Asano, K., Lauterbach, M. \& Mayadas, T.N. Human neutrophil Fcgamma receptors initiate and play specialized nonredundant roles in antibody-mediated inflammatory diseases. Immunity 28, 833-846 (2008).

70. Chauhan, P., Hu, S., Sheng, W.S., Prasad, S. \& Lokensgard, J.R. Modulation of microglial cell fc $\gamma$ receptor expression following viral brain infection. Scientific Reports 7, 41889 (2017).

71. Takaki, R., Watson, S.R. \& Lanier, L.L. DAP12: an adapter protein with dual functionality. Immunological Reviews 214, 118-129 (2006).

72. Lanier, L.L., Corliss, B.C., Wu, J., Leong, C. \& Phillips, J.H. Immunoreceptor DAP12 bearing a tyrosine-based activation motif is involved in activating NK cells. Nature 391, 703-707 (1998).

73. Bouchon, A., Hernández-Munain, C., Cella, M. \& Colonna, M. A DAP12-mediated pathway regulates expression of CC chemokine receptor 7 and maturation of human dendritic cells. The Journal of Experimental Medicine 194, 1111-1122 (2001).
74. Willoughby, J., Griffiths, J., Tews, I. \& Cragg, M.S. OX40: Structure and function - What questions remain? Molecular Immunology 83, 13-22 (2017).

75. Ward-Kavanagh, L.K., Lin, W.W., Šedý, J.R. \& Ware, C.F. The TNF Receptor Superfamily in Co-stimulating and Co-inhibitory Responses. Immunity 44, 1005-1019 (2016).

76. Karulf, M., Kelly, A., Weinberg, A.D. \& Gold, J.A. OX40 ligand regulates inflammation and mortality in the innate immune response to sepsis. Journal of Immunology 185, 4856-4862 (2010).

77. Sano, S. et al. CRISPR-mediated gene editing to assess the roles of TET2 and DNMT3A in clonal hematopoiesis and cardiovascular disease. Circulation Research 123, 335-341 (2018).

78. Bick, A.G. et al. Genetic Interleukin 6 Signaling Deficiency Attenuates Cardiovascular Risk in Clonal Hematopoiesis. Circulation 141, 124-131 (2020).

79. Leoni, C. et al. Dnmt3a restrains mast cell inflammatory responses. Proceedings of the National Academy of Sciences of the United States of America 114, E1490-E1499 (2017).

80. Jaiswal, S. et al. Clonal Hematopoiesis and Risk of Atherosclerotic Cardiovascular Disease. New England Journal of Medicine 377, 111121 (2017).

81. Cull, A.H., Snetsinger, B., Buckstein, R., Wells, R.A. \& Rauh, M.J. Tet2 restrains inflammatory gene expression in macrophages. Experimental Hematology 55, 56-70.e13 (2017).

82. Ma, L. et al. Prosurvival kinase PIM2 is a therapeutic target for eradication of chronic myeloid leukemia stem cells. Proc Natl Acad Sci U S A 116, 10482-10487 (2019).

83. Vester, S.K. et al. Nucleolin acts as the receptor for C1QTNF4 and supports C1QTNF4-mediated innate immunity modulation. The Journal of Biological Chemistry, 100513 (2021).

84. Li, Q. et al. Identification of C1qTNF-related protein 4 as a potential cytokine that stimulates the STAT3 and NF- $\mathrm{KB}$ pathways and promotes cell survival in human cancer cells. Cancer Letters 308, 203-214 (2011).

85. Ramalingam, P. et al. Chronic activation of endothelial MAPK disrupts hematopoiesis via NFKB dependent inflammatory stress reversible by SCGF. Nature Communications 11, 1-20 (2020).

86. Shen, B. et al. Integrin alpha11 is an Osteolectin receptor and is required for the maintenance of adult skeletal bone mass. eLife 8(2019).

87. Ito, C. et al. Serum stem cell growth factor for monitoring hematopoietic recovery following stem cell transplantation. Bone Marrow Transplant 32, 391-8 (2003).

88. Ketkar, S. et al. Remethylation of Dnmt3a (-/-) hematopoietic cells is associated with partial correction of gene dysregulation and reduced myeloid skewing. Proc Natl Acad Sci U S A 117, 3123-3134 (2020).

89. Chitre, S. et al. Clonal hematopoiesis in patients with multiple myeloma undergoing autologous stem cell transplantation. Leukemia 32, 2020-2024 (2018).

90. Gibson, C.J. et al. Clonal hematopoiesis associated with adverse outcomes after autologous stem-cell transplantation for lymphoma. Journal of Clinical Oncology 35, 1598-1605 (2017).

91. Kato, M. et al. Donor cell-derived hematological malignancy: A survey by the Japan Society for Hematopoietic Cell Transplantation. Vol. 30 1742-1745 (Nature Publishing Group, 2016).

92. Soerensen, J.F. et al. Clonal hematopoiesis predicts development of therapy-related myeloid neoplasms post-autologous stem cell transplantation. Blood Advances 4, 885-892 (2020).

93. Wong, W.H. et al. Engraftment of rare, pathogenic donor hematopoietic mutations in unrelated hematopoietic stem cell transplantation. Science translational medicine 12(2020).

94. Wingender, E., Dietze, P., Karas, H. \& Knüppel, R. TRANSFAC: a database on transcription factors and their DNA binding sites. Nucleic Acids Research 24, 238-241 (1996).

95. Liberzon, A. et al. Molecular signatures database (MSigDB) 3.0. Bioinformatics 27, 1739-1740 (2011).

96. Subramanian, A. et al. Gene set enrichment analysis: A knowledgebased approach for interpreting genome-wide expression profiles. Proceedings of the National Academy of Sciences of the United States of America 102, 15545-15550 (2005). 
bioRxiv preprint doi: https://doi.org/10.1101/2022.01.14.476225; this version posted January 16, 2022. The copyright holder for this preprint (which was not certified by peer review) is the author/funder, who has granted bioRxiv a license to display the preprint in perpetuity. It is made available under aCC-BY-NC-ND 4.0 International license.

97. Ben-Porath, I. et al. An embryonic stem cell-like gene expression signature in poorly differentiated aggressive human tumors. Nature Genetics 40, 499-507 (2008).

98. Coller, H.A. et al. Expression analysis with oligonucleotide microarrays reveals that MYC regulates genes involved in growth, cell cycle, signaling, and adhesion. Proceedings of the National Academy of Sciences of the United States of America 97, 3260-3265 (2000).

99. Delgado, M.D. \& León, J. Myc roles in hematopoiesis and leukemia. Genes \& cancer 1, 605-16 (2010).

100. Guo, Y. et al. c-Myc-mediated control of cell fate in megakaryocyteerythrocyte progenitors. Blood 114, 2097-2106 (2009).

101. Mayers, S. et al. Establishment of an erythroid progenitor cell line capable of enucleation achieved with an inducible c-Myc vector. BMC Biotechnology 19, 21-21 (2019).

102. Vaisvila, R. et al. EM-seq: Detection of DNA Methylation at Single Base Resolution from Picograms of DNA. bioRxiv, 2019.12.20.884692 (2019).

103. Picelli, S. et al. Full-length RNA-seq from single cells using Smartseq2. Nat Protoc 9, 171-81 (2014).

104. Spencer, D.H. et al. CpG Island Hypermethylation Mediated by DNMT3A Is a Consequence of AML Progression. Cell 168, 801-816 e13 (2017).

105. Glass, J.L. et al. Epigenetic Identity in AML Depends on Disruption of Nonpromoter Regulatory Elements and Is Affected by Antagonistic Effects of Mutations in Epigenetic Modifiers. Cancer Discov 7, 868-883 (2017).

106. Vaisvila, R. et al. Enzymatic methyl sequencing detects DNA methylation at single-base resolution from picograms of DNA. Genome Res (2021).

107. Wang, J. et al. Double restriction-enzyme digestion improves the coverage and accuracy of genome-wide $\mathrm{CpG}$ methylation profiling by reduced representation bisulfite sequencing. BMC Genomics 14, 11 (2013).

108. Andersson, R. et al. An atlas of active enhancers across human cell types and tissues. Nature 507, 455-461 (2014).

109. Davis, C.A. et al. The Encyclopedia of DNA elements (ENCODE): data portal update. Nucleic Acids Res 46, D794-D801 (2018).

110. Adelman, E.R. et al. Aging Human Hematopoietic Stem Cells Manifest Profound Epigenetic Reprogramming of Enhancers That May Predispose to Leukemia. Cancer Discov 9, 1080-1101 (2019).

111. Heyn, P. et al. Gain-of-function DNMT3A mutations cause microcephalic dwarfism and hypermethylation of Polycombregulated regions. Nature Genetics 51, 96-105 (2019).

112. Jeong, M. et al. Large conserved domains of low DNA methylation maintained by Dnmt3a. Nat Genet 46, 17-23 (2014).

113. Zhang, X. et al. Large DNA Methylation Nadirs Anchor Chromatin Loops Maintaining Hematopoietic Stem Cell Identity. Mol Cell 78, 506-521 e6 (2020).

114. Hawkins, R.D. et al. Distinct epigenomic landscapes of pluripotent and lineage-committed human cells. Cell Stem Cell 6, 479-491 (2010).

115. Margueron, R. \& Reinberg, D. The Polycomb complex PRC2 and its mark in life. Nature 469, 343-349 (2011).

116. Mohn, F. et al. Lineage-specific polycomb targets and de novo DNA methylation define restriction and potential of neuronal progenitors. Molecular Cell 30, 755-766 (2008).

117. Xie, H. et al. Polycomb repressive complex 2 regulates normal hematopoietic stem cell function in a developmental-stage-specific manner. Cell Stem Cell 14, 68-80 (2014).

118. Cancer Genome Atlas Research, N. et al. Genomic and epigenomic landscapes of adult de novo acute myeloid leukemia. $N$ Engl J Med 368, 2059-74 (2013).

119. Emperle, M. et al. Mutations of R882 change flanking sequence preferences of the DNA methyltransferase DNMT3A and cellular methylation patterns. Nucleic acids research 47, 11355-11367 (2019).

120. Anteneh, H., Fang, J. \& Song, J. Structural basis for impairment of DNA methylation by the DNMT3A R882H mutation. Nat Commun 11, 2294 (2020).
121. Grau, J., Schmidt, F. \& Schulz, M.H. Widespread effects of DNA methylation and intra-motif dependencies revealed by novel transcription factor binding models. bioRxiv (2020).

122. Yin, Y. et al. Impact of cytosine methylation on DNA binding specificities of human transcription factors. Science 356(2017).

123. Mariani, C.J. et al. TET1-mediated hydroxymethylation facilitates hypoxic gene induction in neuroblastoma. Cell Rep 7, 1343-1352 (2014).

124. Takubo, K. et al. Regulation of the HIF-1alpha level is essential for hematopoietic stem cells. Cell Stem Cell 7, 391-402 (2010).

125. Krock, B.L. et al. The aryl hydrocarbon receptor nuclear translocator is an essential regulator of murine hematopoietic stem cell viability. Blood 125, 3263-3272 (2015).

126. Forristal, C.E. et al. Pharmacologic stabilization of HIF-1 $\alpha$ increases hematopoietic stem cell quiescence in vivo and accelerates blood recovery after severe irradiation. Blood 121, 759-769 (2013).

127. Forristal, C.E. et al. HIF- $1 \alpha$ is required for hematopoietic stem cell mobilization and 4-prolyl hydroxylase inhibitors enhance mobilization by stabilizing HIF-1 $\alpha$. Leukemia 29, 1366-1378 (2015).

128. Forristal, C.E. \& Levesque, J.-P. Targeting the hypoxia-sensing pathway in clinical hematology. Stem cells translational medicine $\mathbf{3}$, 135-140 (2014).

129. Adelman, D.M., Maltepe, E. \& Simon, M.C. Multilineage embryonic hematopoiesis requires hypoxic ARNT activity. Genes \& Development 13, 2478-2483 (1999).

130. Takubo, K. et al. Regulation of glycolysis by Pdk functions as a metabolic checkpoint for cell cycle quiescence in hematopoietic stem cells. Cell Stem Cell 12, 49-61 (2013).

131. Li, Y. et al. Setd1a and NURF mediate chromatin dynamics and gene regulation during erythroid lineage commitment and differentiation. Nucleic Acids Research 44, 7173-7188 (2016).

132. Huang, S., Li, X., Yusufzai, T.M., Qiu, Y. \& Felsenfeld, G. USF1 recruits histone modification complexes and is critical for maintenance of a chromatin barrier. Molecular and Cellular Biology 27, 7991-8002 (2007).

133. Grosselin, K. et al. High-throughput single-cell ChIP-seq identifies heterogeneity of chromatin states in breast cancer. Nat Genet 51, 1060-1066 (2019).

134. Rotem, A. et al. Single-cell ChIP-seq reveals cell subpopulations defined by chromatin state. Nat Biotechnol 33, 1165-72 (2015).

135. Wang, Q. et al. CoBATCH for High-Throughput Single-Cell Epigenomic Profiling. Mol Cell 76, 206-216 e7 (2019).

136. Fu, L. et al. Predicting transcription factor binding in single cells through deep learning. Science Advances 6(2020).

137. Ugarte, F. et al. Progressive chromatin condensation and H3K9 methylation regulate the differentiation of embryonic and hematopoietic stem cells. Stem cell reports 5, 728-740 (2015).

138. Martin, E.W. et al. Chromatin accessibility maps provide evidence of multilineage gene priming in hematopoietic stem cells. Epigenetics \& Chromatin 14, 2 (2021).

139. Prendergast, G.C. \& Ziff, E.B. Methylation-sensitive sequencespecific DNA binding by the c-Myc basic region. Science 251, 186-9 (1991).

140. Tate, P.H. \& Bird, A.P. Effects of DNA methylation on DNA-binding proteins and gene expression. Curr Opin Genet Dev 3, 226-31 (1993).

141. Granja, J.M. et al. Single-cell multiomic analysis identifies regulatory programs in mixed-phenotype acute leukemia. Nature Biotechnology 37, 1458-1465 (2019).

142. Setty, M. et al. Characterization of cell fate probabilities in singlecell data with Palantir. Nature Biotechnology 37, 451-460 (2019).

143. Stoeckius, M. et al. Simultaneous epitope and transcriptome measurement in single cells. Nat Methods 14, 865-868 (2017).

144. Tovy, A. et al. Tissue-Biased Expansion of DNMT3A-Mutant Clones in a Mosaic Individual Is Associated with Conserved Epigenetic Erosion. Cell Stem Cell 27, 326-335.e4 (2020).

145. Zavidij, O. et al. Single-cell RNA sequencing reveals compromised immune microenvironment in precursor stages of multiple myeloma. Nature Cancer 1, 493-506 (2020). 
bioRxiv preprint doi: https://doi.org/10.1101/2022.01.14.476225; this version posted January 16, 2022. The copyright holder for this preprint (which was not certified by peer review) is the author/funder, who has granted bioRxiv a license to display the preprint in perpetuity. It is made available under aCC-BY-NC-ND 4.0 International license.

146. Caiado, F., Pietras, E.M. \& Manz, M.G. Inflammation as a regulator of hematopoietic stem cell function in disease, aging, and clonal selection. J Exp Med 218(2021).

147. Bintu, L. et al. Dynamics of epigenetic regulation at the single-cell level. Science 351, 720-724 (2016).

148. Asenjo, H.G. et al. Polycomb regulation is coupled to cell cycle transition in pluripotent stem cells. Science Advances 6, eaay4768 (2020).

149. Richly, H., Aloia, L. \& Di Croce, L. Roles of the Polycomb group proteins in stem cells and cancer. Cell death \& disease 2, e204 (2011).

150. Zepeda-Martinez, J.A. et al. Parallel PRC2/cPRC1 and vPRC1 pathways silence lineage-specific genes and maintain self-renewal in mouse embryonic stem cells. Science Advances 6, eaax5692 (2020).

151. Voigt, P. et al. Asymmetrically modified nucleosomes. Cell 151, 18193 (2012).

152. Shema, E. et al. Single-molecule decoding of combinatorially modified nucleosomes. Science 352, 717-21 (2016).

153. Lessard, J. \& Sauvageau, G. Bmi-1 determines the proliferative capacity of normal and leukaemic stem cells. Nature 423, 255-60 (2003).

154. Liu, Y. et al. Convergence of oncogenic cooperation at single-cell and single-gene levels drives leukemic transformation. Nat Commun 12, 6323 (2021).

155. Panopoulos, A.D. \& Watowich, S.S. Granulocyte colony-stimulating factor: molecular mechanisms of action during steady state and 'emergency' hematopoiesis. Cytokine 42, 277-88 (2008).

156. Bernitz, J.M., Daniel, M.G., Fstkchyan, Y.S. \& Moore, K. Granulocyte colony-stimulating factor mobilizes dormant hematopoietic stem cells without proliferation in mice. Blood 129, 1901-1912 (2017).

157. Lu, R. et al. A Model System for Studying the DNMT3A Hotspot Mutation (DNMT3A(R882)) Demonstrates a Causal Relationship between Its Dominant-Negative Effect and Leukemogenesis. Cancer Res 79, 3583-3594 (2019).

158. van Galen, P. et al. The unfolded protein response governs integrity of the haematopoietic stem-cell pool during stress. Nature 510, 268-72 (2014).

159. Rodriguez-Meira, A. et al. Unravelling Intratumoral Heterogeneity through High-Sensitivity Single-Cell Mutational Analysis and Parallel RNA Sequencing. Mol Cell 73, 1292-1305 e8 (2019).

160. Petti, A.A. et al. A general approach for detecting expressed mutations in AML cells using single cell RNA-sequencing. Nat Commun 10, 3660 (2019).

161. Ludwig, L.S. et al. Lineage Tracing in Humans Enabled by Mitochondrial Mutations and Single-Cell Genomics. Cell 176, 13251339 e22 (2019).
162. Gothert, J.R. et al. In vivo fate-tracing studies using the Scl stem cell enhancer: embryonic hematopoietic stem cells significantly contribute to adult hematopoiesis. Blood 105, 2724-32 (2005).

163. Butler, A., Hoffman, P., Smibert, P., Papalexi, E. \& Satija, R. Integrating single-cell transcriptomic data across different conditions, technologies, and species. Nature Biotechnology 36, 411-420 (2018).

164. McInnes, L., Healy, J. \& Melville, J. UMAP: Uniform Manifold Approximation and Projection for Dimension Reduction. (2018).

165. Tange, O. GNU Parallel 20200622 ('Privacy Shield'). (2020).

166. Bolker, B.M. et al. Generalized linear mixed models: a practical guide for ecology and evolution. Trends Ecol Evol 24, 127-35 (2009).

167. Yu, G., Wang, L.-G., Han, Y. \& He, Q.-Y. clusterProfiler: an R package for comparing biological themes among gene clusters. Omics : a journal of integrative biology 16, 284-287 (2012).

168. Müller, S., Cho, A., Liu, S.J., Lim, D.A. \& Diaz, A. CONICS integrates scRNA-seq with DNA sequencing to map gene expression to tumor sub-clones. Bioinformatics 34, 3217-3219 (2018).

169. Bagnoli, J.W. et al. Sensitive and powerful single-cell RNA sequencing using mcSCRB-seq. Nature Communications 9 , 1-8 (2018).

170. Van de Sande, B. et al. A scalable SCENIC workflow for single-cell gene regulatory network analysis. Nature Protocols 15, 2247-2276 (2020).

171. Mendenhall, E.M. et al. GC-rich sequence elements recruit PRC2 in mammalian ES cells. PLoS Genet 6, e1001244 (2010).

172. Li, H. et al. Polycomb-like proteins link the PRC2 complex to CpG islands. Nature 549, 287-291 (2017).

173. Saxonov, S., Berg, P. \& Brutlag, D.L. A genome-wide analysis of CpG dinucleotides in the human genome distinguishes two distinct classes of promoters. Proceedings of the National Academy of Sciences of the United States of America 103, 1412-1417 (2006).

174. Granja, J.M. et al. ArchR is a scalable software package for integrative single-cell chromatin accessibility analysis. Nature Genetics 53, 403-411 (2021).

175. Popescu, D.-M. et al. Decoding human fetal liver haematopoiesis. Nature 574, 365-371 (2019).

176. McCarthy, D.J., Campbell, K.R., Lun, A.T.L. \& Wills, Q.F. Scater: preprocessing, quality control, normalization and visualization of single-cell RNA-seq data in R. Bioinformatics 33, 1179-1186 (2017).

177. Schep, A.N., Wu, B., Buenrostro, J.D. \& Greenleaf, W.J. chromVAR: inferring transcription-factor-associated accessibility from singlecell epigenomic data. Nature Methods 14, 975-978 (2017). 
bioRxiv preprint doi: https://doi.org/10.1101/2022.01.14.476225; this version posted January 16, 2022. The copyright holder for this preprint (which was not certified by peer review) is the author/funder, who has granted bioRxiv a license to display the preprint in perpetuity. It is made available under aCC-BY-NC-ND 4.0 International license.

\section{EXTENDED DATA}

a

\begin{tabular}{ccccccc} 
Patient ID & Mutation & Cell \# Cells genotyped (\%) & Mutant cells (\%) & $\begin{array}{c}\text { Bulk VAF in } \\
\text { unsorted stem cell grafts }\end{array}$ & $\begin{array}{c}\text { Additional mutations } \\
\text { (VAF) }\end{array}$ \\
\hline CH01 & DNMT3A-R882C & 6133 & 24 & 17 & 0.13 & - \\
\hline CH02 & DNMT3A-R882C & 5372 & 18 & 50 & 0.34 & - \\
\hline CH03 & DNMT3A-R882H & 7379 & 26 & 41 & 0.16 & TET2 p.Q706* (0.5) \\
\hline CH04 & DNMT3A-R882C & 8440 & 25 & 13 & 0.09 & - \\
\hline
\end{tabular}

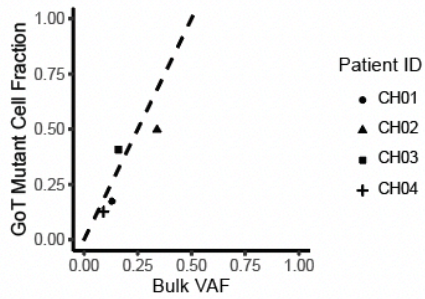

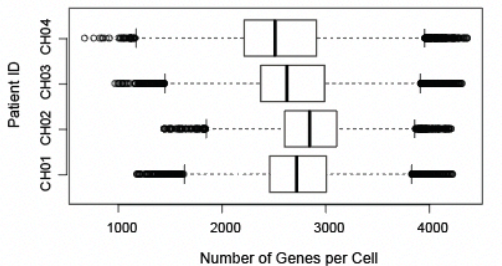

d

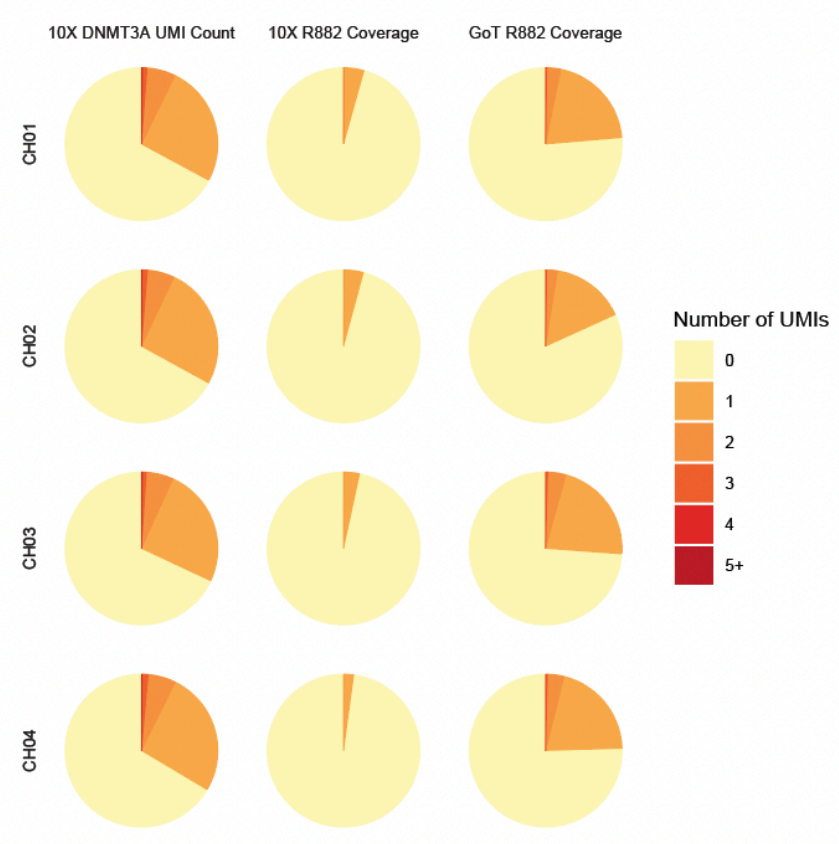

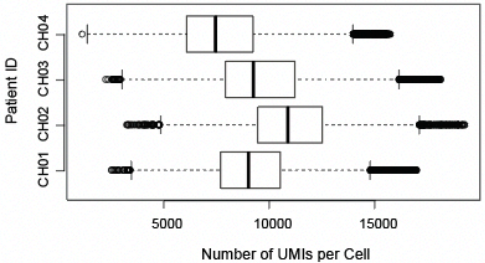

e
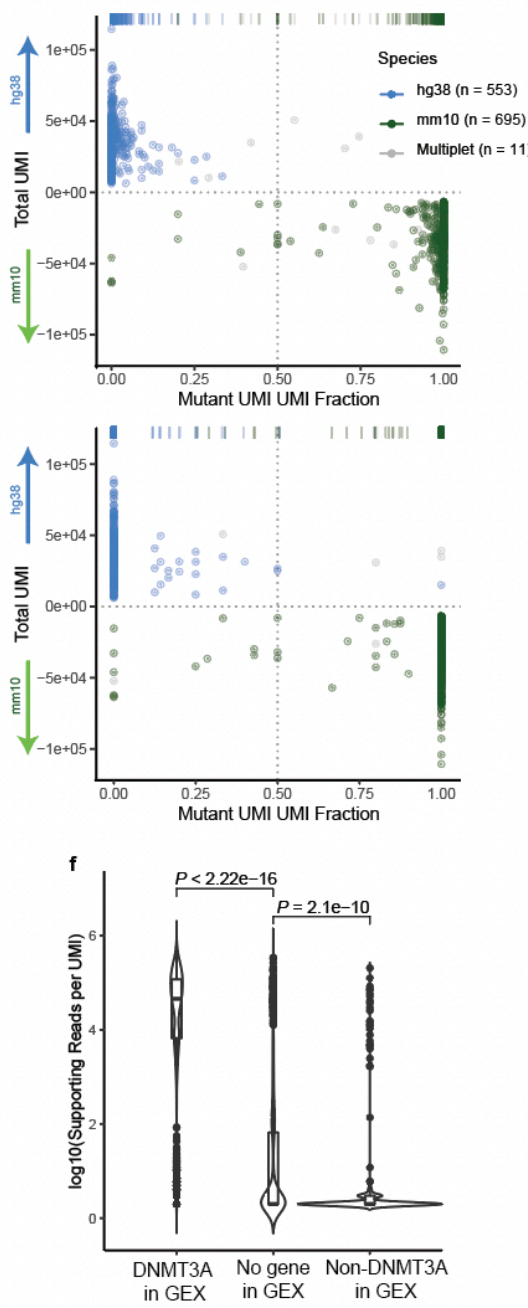

Extended Data Figure 1. GoT captures genotyping information of thousands of CD34+ cells in scRNA-seq. a, Summary of GoT data from CH patient samples with DNMT3A R882 mutations. b, Number of genes per cell (left) and number of UMIs per cell (right) from CD34+ sorted hematopoietic progenitors by patient sample after QC filters. c, DNMT3A R882 mutant fraction of single cells determined by GoT versus DNMT3A R882 mutation variant allele frequencies (VAF) in bulk sequencing of matched unsorted stem cell product. d, Fraction of cells by number of DNMT3A UMIs in standard 10x Genomics data without genotyping information (left), DNMT3A UMIs with R882 locus coverage in standard 10x data (middle), and DNMT3A UMIs with R882 locus coverage in GoT amplicon library (right). e, Species-mixing experiment data in which mouse cells (Ba/F3) with a human mutant CALR transgene were mixed with human cells (UT-7) with a human wildtype CALR transgene. Mouse and human genome alignment of 10x data with genotyping data from GoT pre (top) and post (bottom) implementation of UMI consensus assembly based on Levenshtein distance (online methods). f, Number of duplicate reads supporting cell barcode-UMI pair in the GoT library that is identified in the 10x gene expression (GEX) library as a DNMT3A gene (left), no gene (middle), or a non-DNMT3A gene (right). 
bioRxiv preprint doi: https://doi.org/10.1101/2022.01.14.476225; this version posted January 16, 2022. The copyright holder for this preprint (which was not certified by peer review) is the author/funder, who has granted bioRxiv a license to display the preprint in perpetuity. It is made available under aCC-BY-NC-ND 4.0 International license.

a

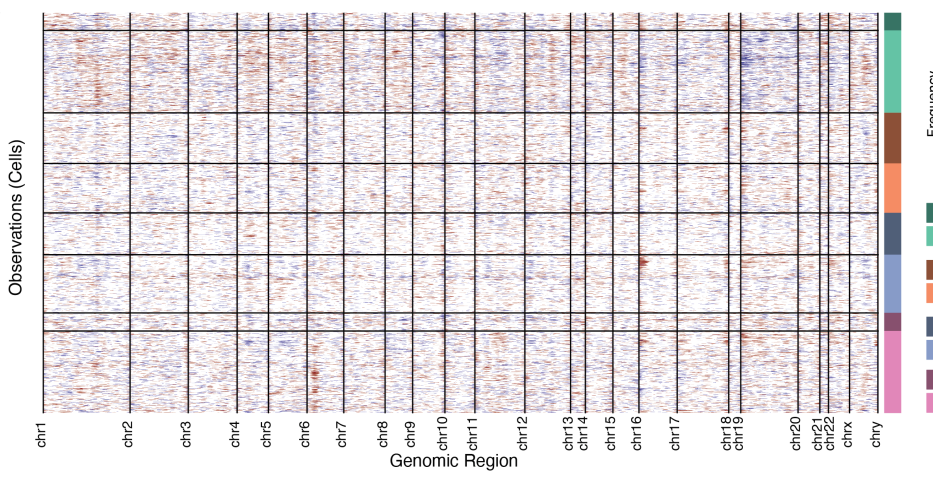

b

Distribution of

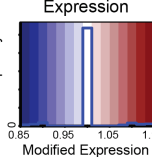

CH01 MUT $(n=179)$ CH01 WT $(n=799)$

CHO2 MUT $(n=487)$ CHO2 WT $(n=491)$

CH03 MUT $(n=397)$ CHO3 WT $(n=581)$ CH04 MUT $(n=122)$
CH04 WT $(n=856)$

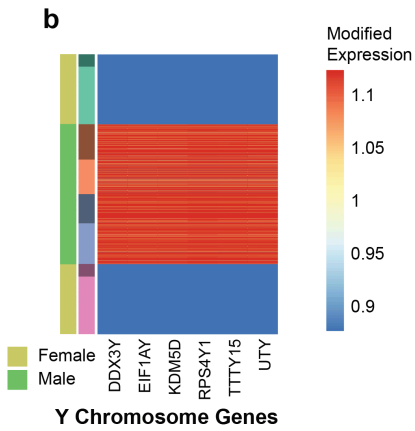

Extended Data Figure 2. Copy number analysis of wildtype and mutant single cells from clonal hematopoiesis patient samples with DNMT3A R882 mutations. a, Heatmap of relative expression of genes ordered by chromosome/chromosomal position following copy number variation analysis using the InferCNV package. Cells (y-axis) are stratified by patient and DNMT3A R882 genotype status. b, Heatmap of relative expression of Y-chromosome genes following copy number variation analysis and cell stratification as in $\mathbf{a}$. 
bioRxiv preprint doi: https://doi.org/10.1101/2022.01.14.476225; this version posted January 16, 2022. The copyright holder for this preprint (which was not certified by peer review) is the author/funder, who has granted bioRxiv a license to display the preprint in perpetuity. It is made available under aCC-BY-NC-ND 4.0 International license.
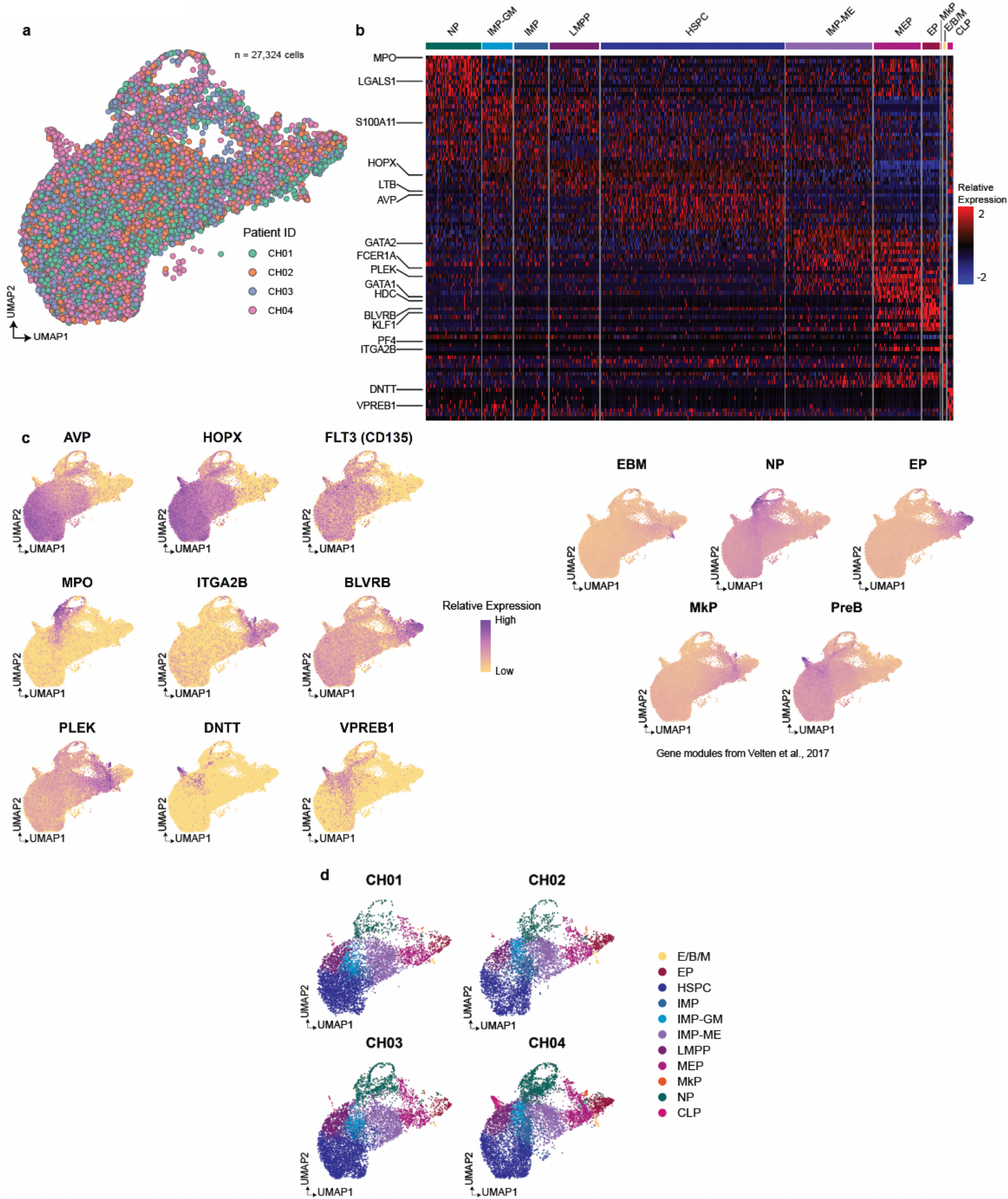

Gene modules from Velten et al, 2017

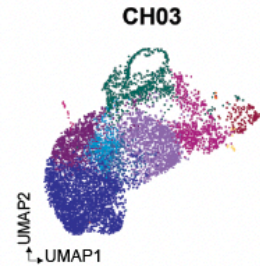

$\rightarrow$ UMAP1

Extended Data Figure 3. Integration of DNMT3A R882 mutation and assignment of progenitor subsets in clonal hematopoiesis patient samples. a, UMAP of $\mathrm{CD}_{3} 4^{+}$progenitor cells from samples $\mathrm{CH01-CH04}$ after integration using the Seurat package (online methods). b, Heatmap of top 10 differentially expressed genes for progenitor subsets. c, Lineage-specific genes (left) and modules from Velten et al. (right, Supplementary Table 2) are scored and projected onto the UMAP representation of $\mathrm{CD} 34^{+}$cells. d, UMAP of $\mathrm{CD} 34^{+}$cells overlaid with cluster assignments, split by patient sample. 
a

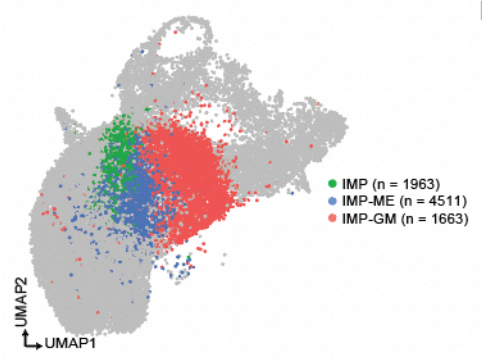

b

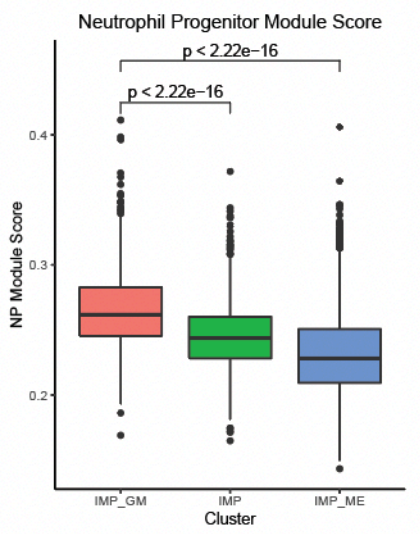

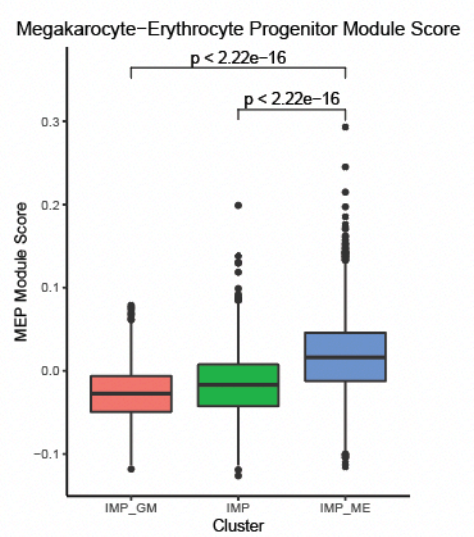

c

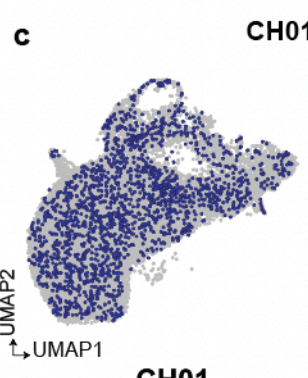

$\mathrm{CH} 01$
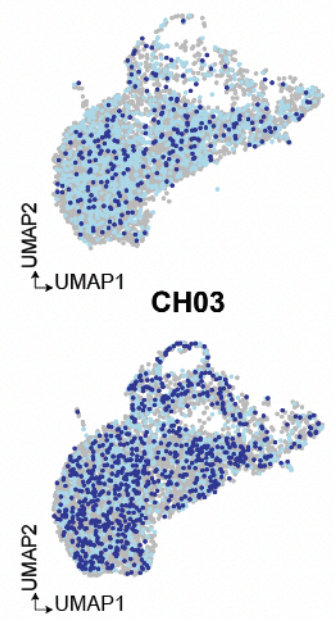

$\mathrm{CH} 01-\mathrm{CH} 04$
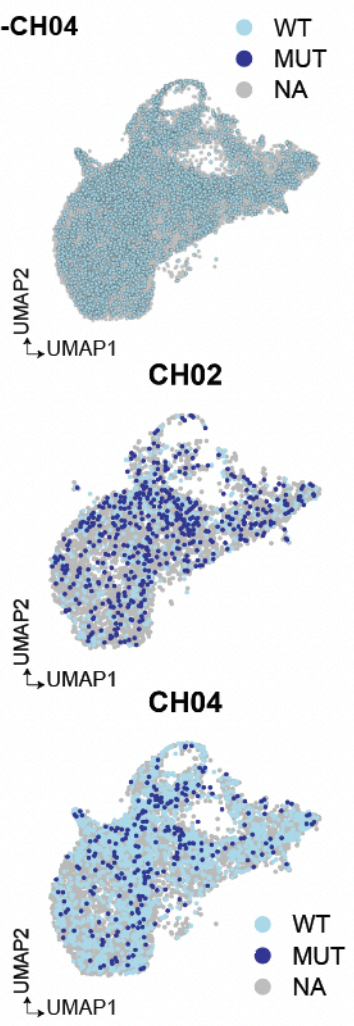
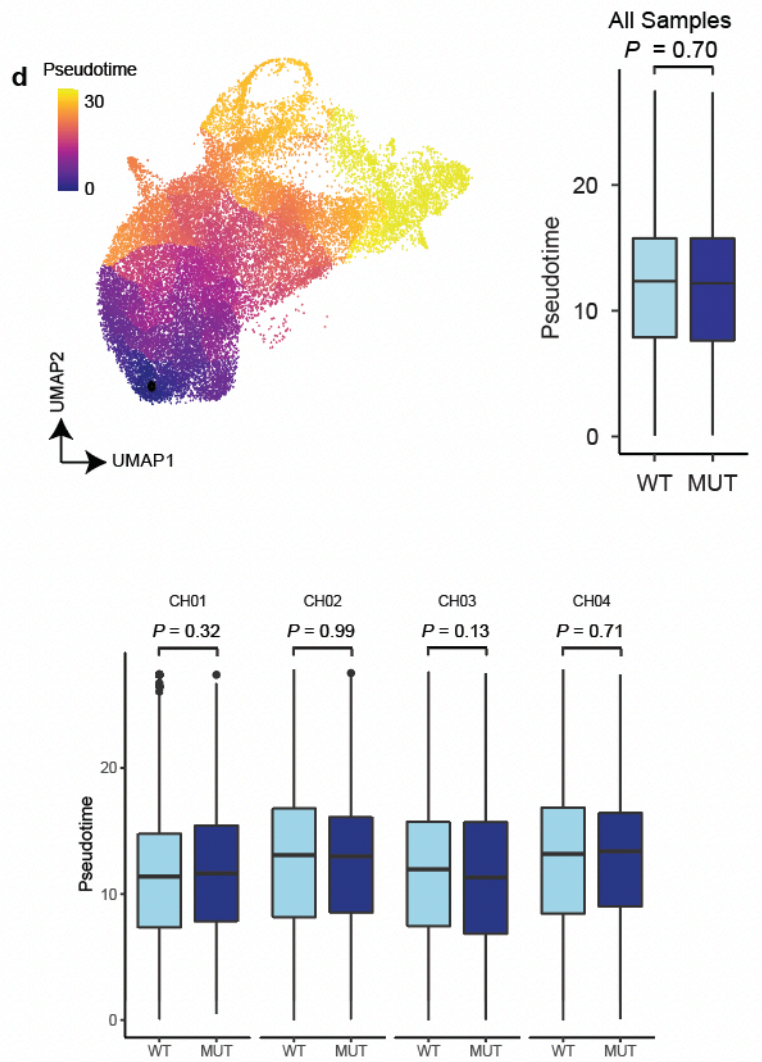

Extended Data Figure 4. Classification of IMPs showing lineage biases and pseudotime analysis between mutated and wildtype cells. a, UMAP of CD34+ cells, overlaid with cluster assignment of all IMP subsets in the dataset. b, Neutrophil and Megakaryocytic-Erythroid lineage specific gene module scores from Velten et al. compared across the three IMP clusters. Pvalue was calculated from Wilcoxon rank sum test. c, UMAP of CD34+ cells overlaid with mutation status for WT, DNMT3A R882 mutant (MUT), or unassigned (NA), split by genotype for all samples (top) and by patient sample (bottom). d, UMAP with projected pseudotime values (top left). Pseudotime comparison between WT and MUT cells for all samples (top right) and for individual samples (bottom) as estimated by Monocle. P-value was calculated from likelihood ratio test of linear mixed model with/without mutation status for aggregate analysis (online methods, top) and Wilcoxon rank sum test for individual samples (bottom). 
bioRxiv preprint doi: https://doi.org/10.1101/2022.01.14.476225; this version posted January 16,2022 . The copyright holder for this preprint (which was not certified by peer review) is the author/funder, who has granted bioRxiv a license to display the preprint in perpetuity. It is made available under aCC-BY-NC-ND 4.0 International license.

a
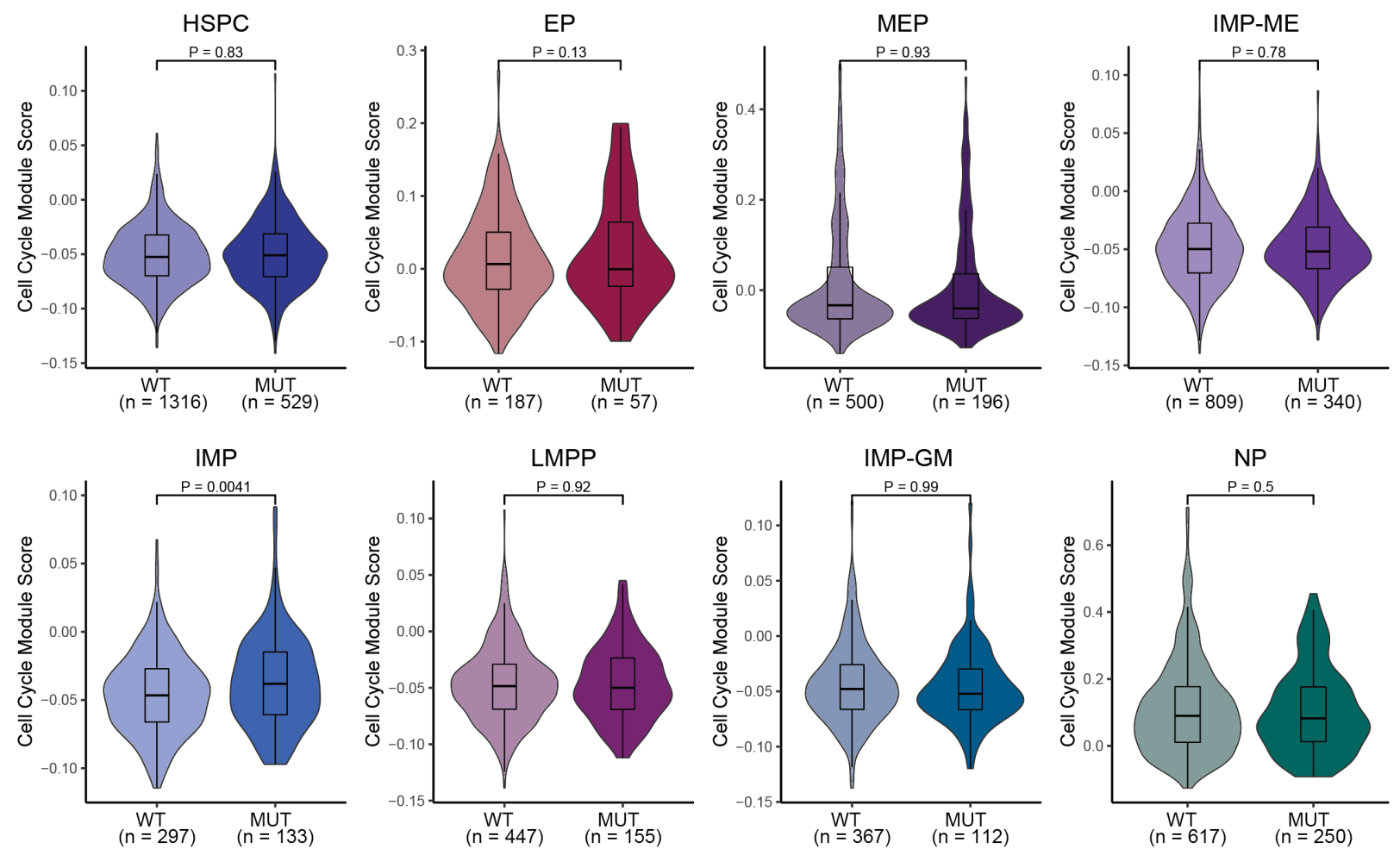

Extended Data Figure 5. Cell cycle module expression comparison between mutated and wildtype progenitor cells. a, Cell cycle module score represents the union of S-phase and G2M-phase gene-module expression (Supplementary Table 2). Pvalue was calculated from likelihood ratio test of linear mixed model with/without mutation status (online methods). Analysis was performed for clusters with at least 200 genotyped cells across all patient samples. 
a

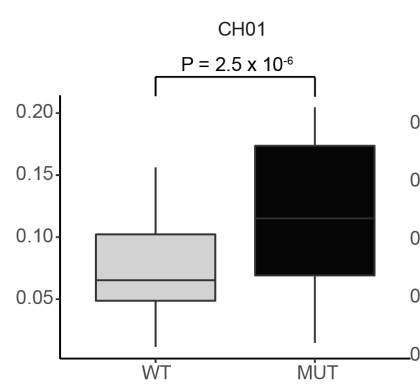

Cummulative probability of IMP to IMP-ME transition

$\mathrm{CH} 02$

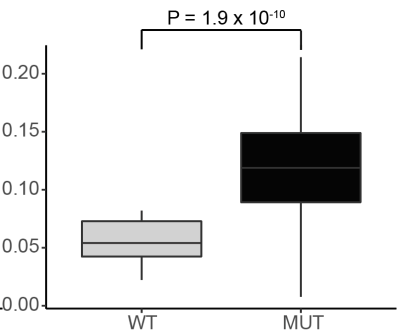

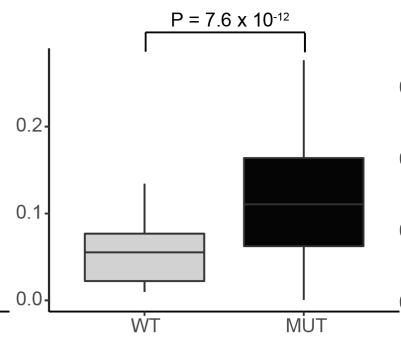

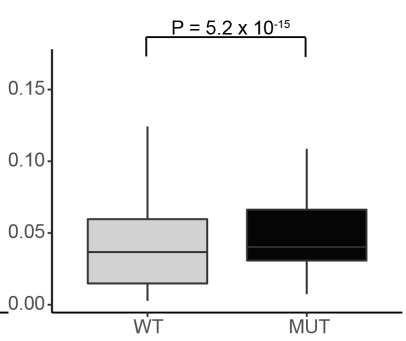

b
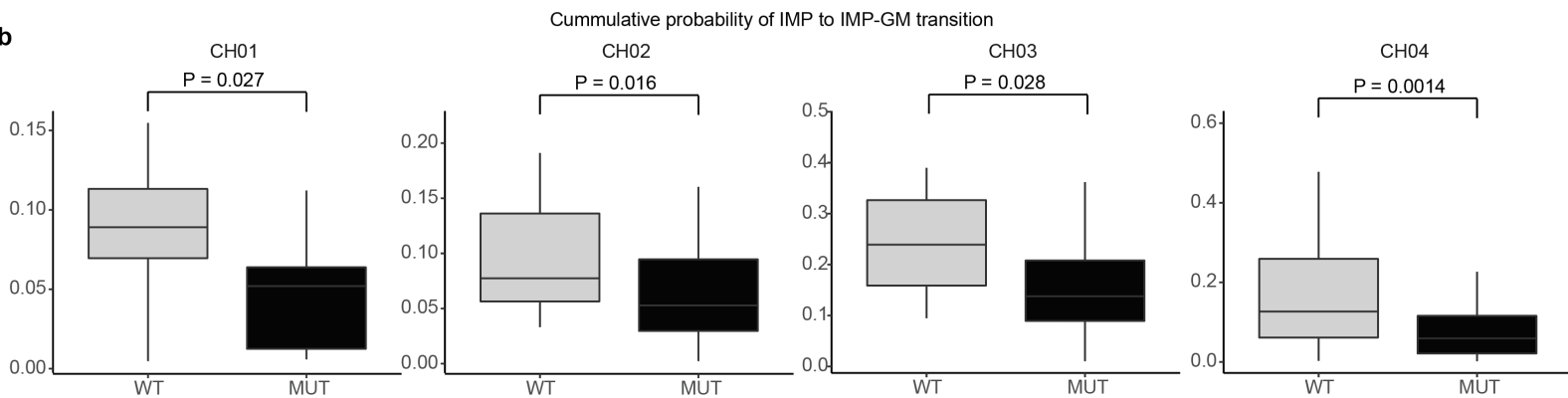

Extended Data Figure 6. Transition probabilities via RNA velocity reveals a megakaryocytic-erythroid bias of IMPs. a, Single cell mean IMP $\rightarrow$ IMP-ME and $\mathbf{b}$, IMP $\rightarrow$ IMP-GM transition probabilities, as measured via RNA velocity, between wildtype or DNMT3A R882 mutant IMPs for each sample. P-values from Wilcoxon rank-sum test. 
bioRxiv preprint doi: https://doi.org/10.1101/2022.01.14.476225; this version posted January 16,2022 . The copyright holder for this preprint (which was not certified by peer review) is the author/funder, who has granted bioRxiv a license to display the preprint in perpetuity. It is made available under aCC-BY-NC-ND 4.0 International license.

a
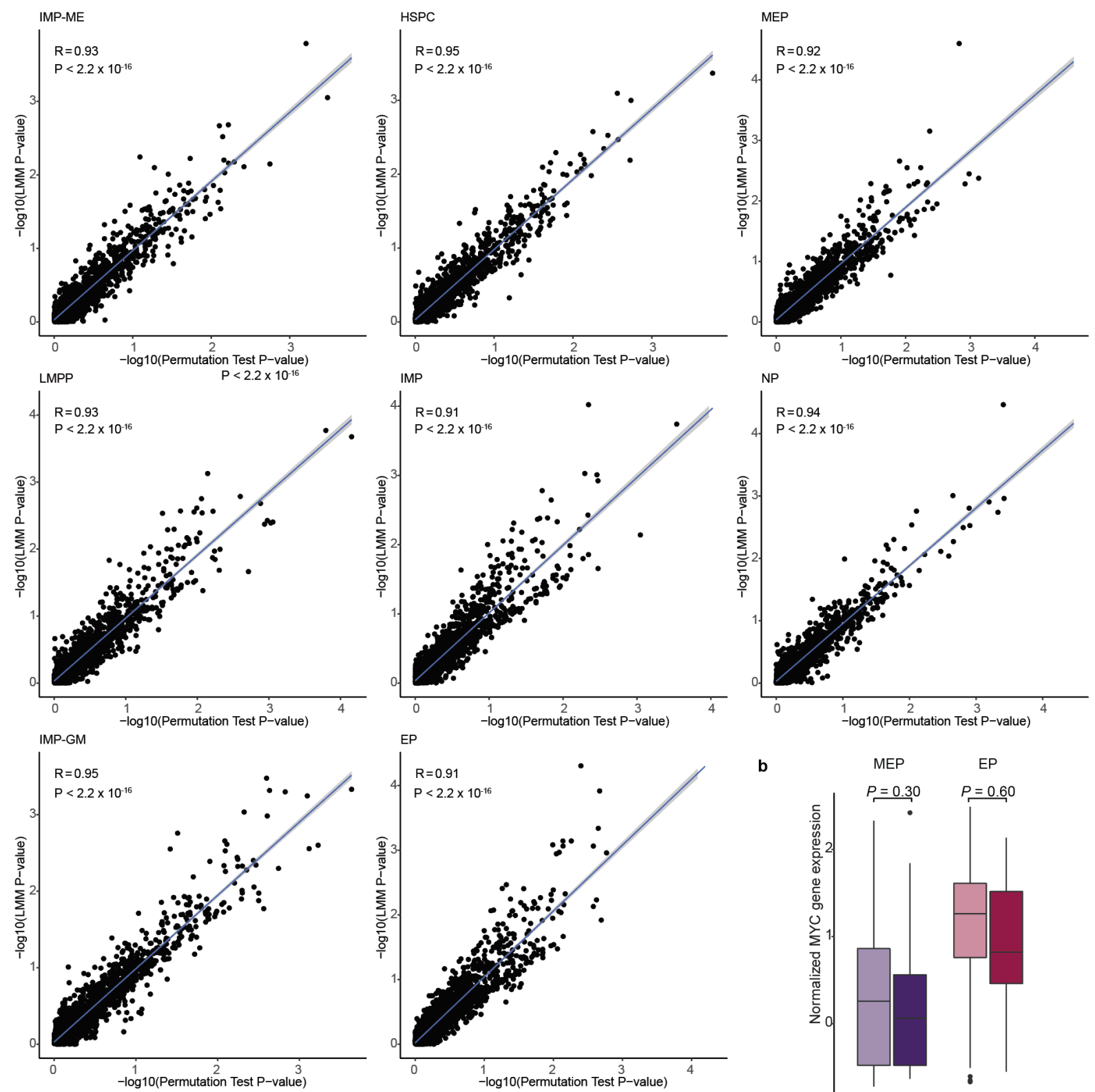

b

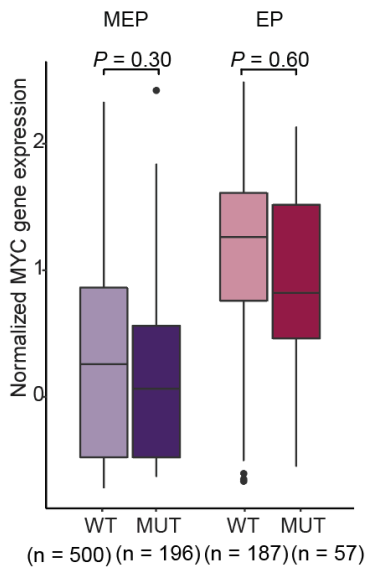

Extended Data Figure 7. Comparison of differential expression analysis between permutation test and linear mixed model and MYC gene expression. a, P-values from permutation test and linear mixed model (online methods) are plotted per gene. Correlation coefficient $\mathrm{R}$ calculated using Pearson's Correlation. P-values derived from Student's t-distribution. b, Normalized MYC gene expression between mutated and wildtype cells in MEP and EP. P-value was calculated from likelihood ratio test of linear mixed model with/without mutation status (online methods). 
bioRxiv preprint doi: https://doi.org/10.1101/2022.01.14.476225; this version posted January 16, 2022. The copyright holder for this preprint (which was not certified by peer review) is the author/funder, who has granted bioRxiv a license to display the preprint in perpetuity. It is made available under aCC-BY-NC-ND 4.0 International license.

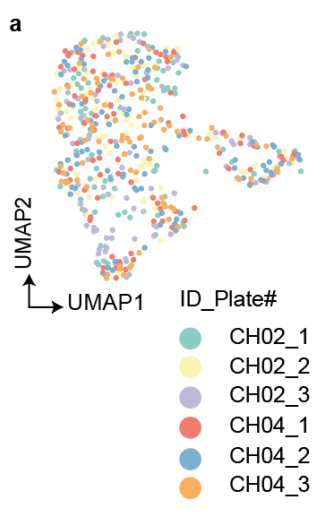

a

d

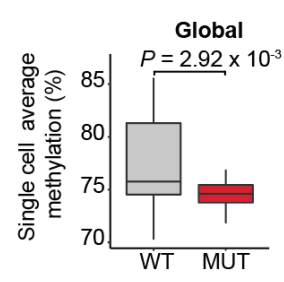

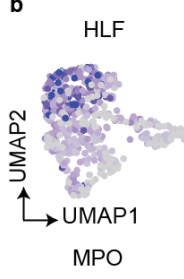
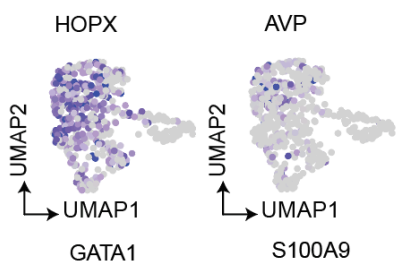

c

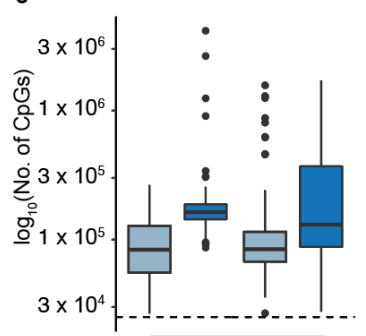

Digestion[ Single Double Single Double

e

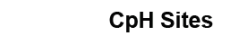

CpH Sites
$P=0.12$

f Hypomethylated TSS
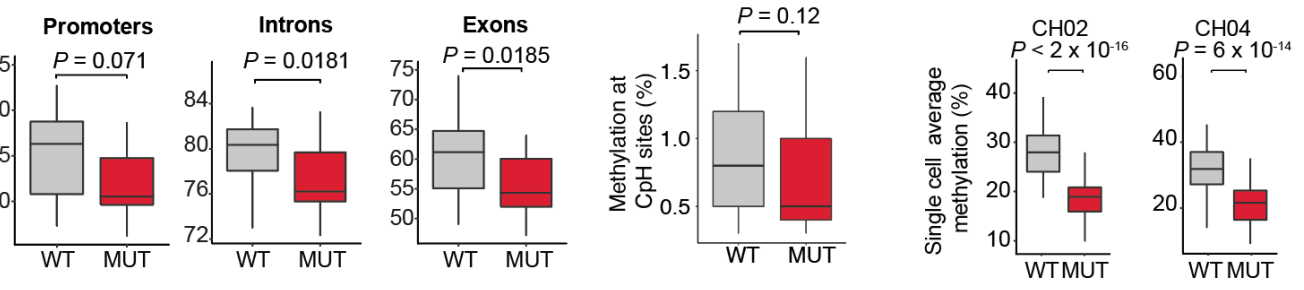

g

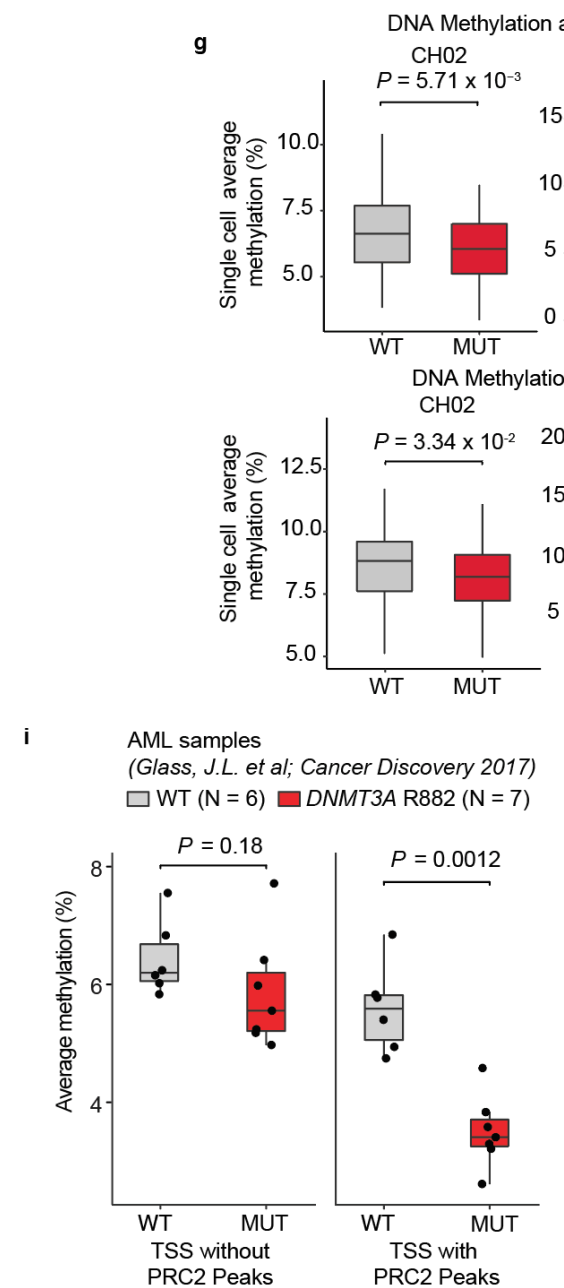

DNA Methylation at SUZ12 Peaks

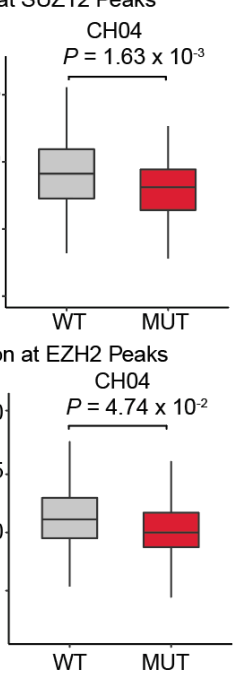

h

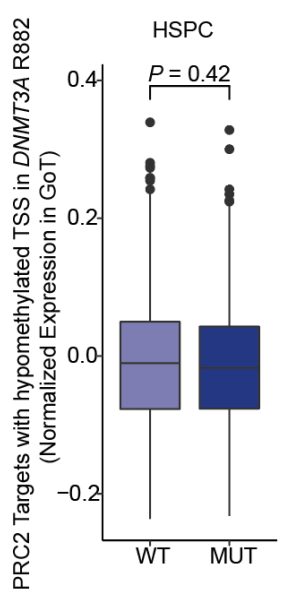

j AML samples

The Cancer Genome Atlas

$\square \mathrm{WT}(\mathrm{N}=122) \square D N M T 3 A \mathrm{R} 882(\mathrm{~N}=9)$

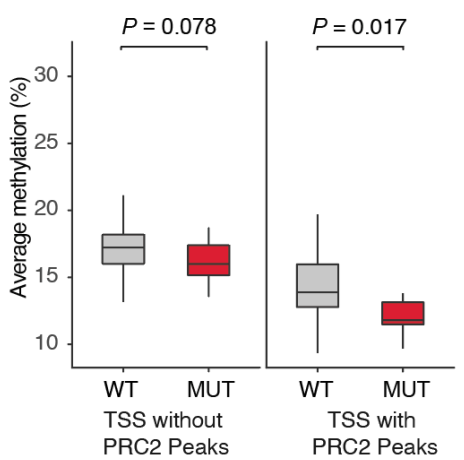

Extended Data Figure 8. Multi-omics single cell methylome, transcriptomic, and somatic genotyping reveals hypomethylation of PRC2 targets in DNMT3A R882 CH. a, UMAP dimensionality reduction ( $\mathrm{n}=528$ cells) based on scRNAseq data (Smart-seq2) after integration and batch correction of six plates (online methods). b, UMAP dimensionality reduction showing cluster gene markers for the transcriptome data. c, Number of CpG sites captured per cell after quality filtering (online methods). The metrics for each sample according to enzymatic digestion with Msp1 (Single) or Msp1 plus HaelII (Double) are shown. d, Average single cell methylation at all regions (global, double digest), promoters, introns or exons. P-values from likelihood ratio test of LMM with/without mutation status (online methods). e, Average single cell methylation at $\mathrm{CpH}$ (i.e. $\mathrm{CpA}$ 
or CpT) sites. f, Average single cell methylation at 269 hypomethylated promoters identified with DMR analysis (shown in Fig. 4e, promoters with P-value $<0.05$ and at least $-5 \%$ methylation change) in $\mathrm{CH} 02$ and $\mathrm{CH} 04$. g, Average single cell methylation at SUZ12 (top panel) and EZH2 (bottom panel) ENCODE ChIP-seq peaks intersected with bivalently H3K27me3, H3K4me3marked regions in $\mathrm{CD}_{3} 4^{+}$cells for $\mathrm{CHO} 2$ and $\mathrm{CH0} 4$. P-values from likelihood ratio test of LMM with/without mutation status. $\mathbf{h}$, Normalized expression of PRC2 target genes with preferentially hypomethylated TSS (from Fig. 4e) in GoT data of WT versus MUT HSPCs. P-values from likelihood ratio test of LMM with/without mutation status. i, Comparison of average methylation values for TSS $\pm 1 \mathrm{~kb}$ regions in DNMT3A WT $(\mathrm{n}=6)$ versus DNMT3A R882, NPM1 mutated acute myeloid leukemia (AML; $\mathrm{n}=$ 7) samples in regions without (left) or with (right) PRC2 ChIP-seq peaks, controlling for CpG content. j, Comparison of average methylation values for promoter regions in WT $(\mathrm{n}=122)$ versus DNMT3A R882 mutated AML $(\mathrm{n}=9)$ samples from TCGA in regions without (left) or with (right) PRC2 ChIP-seq peaks, controlling for CpG content. 
bioRxiv preprint doi: https://doi.org/10.1101/2022.01.14.476225; this version posted January 16, 2022. The copyright holder for this preprint (which was not certified by peer review) is the author/funder, who has granted bioRxiv a license to display the preprint in perpetuity. It is made available under aCC-BY-NC-ND 4.0 International license.
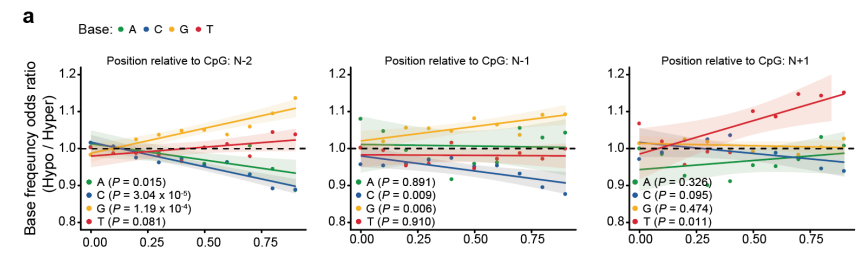

Minimum CpG methylation difference (DNMT3A R878 - WT)
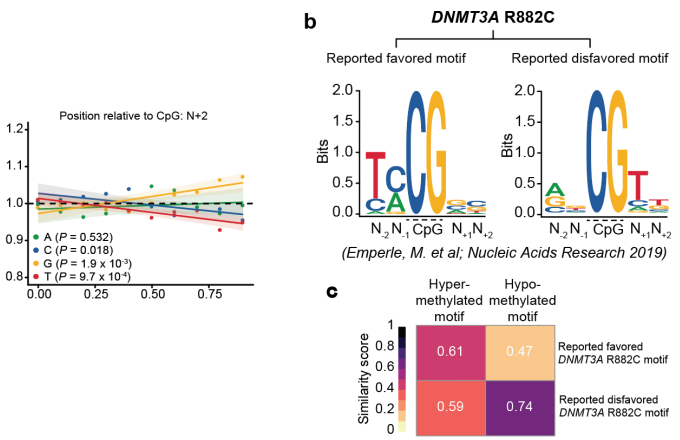
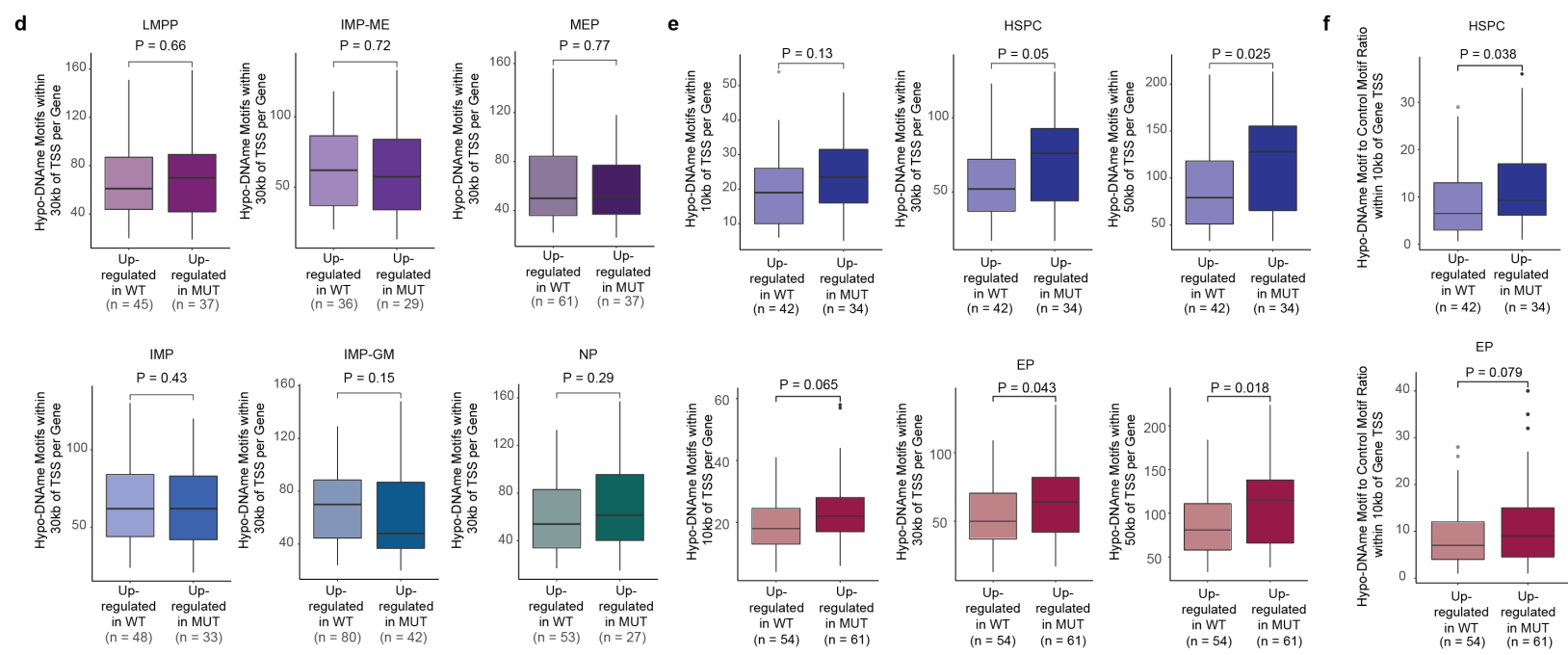

Extended Data Figure 9. Motif enrichment at hypomethylated CpGs and hypomethylated motif enrichment in regions around differentially expressed genes. a, Base frequency odds ratio of hypo- versus hyper-methylated CpG flanking sequences at positions $\mathrm{N}-2, \mathrm{~N}-1, \mathrm{~N}+1$, and $\mathrm{N}+2$. The odds ratios were derived from base frequencies of flanking positions of the CpG sites hypo- or hyper-methylated in mutant versus wildtype cells above the thresholds shown in the $\mathrm{x}$ axis for minimum absolute CpG methylation difference (Pearson correlation, P-values derived from F-test). b, Reported motif logos derived from Emperle et al. for either hypomethylated (disfavored) or hypermethylated (favored) sites for DNMT3A R882 compared to its wildtype counterpart (left). c, Similarity scores between the reported and our de novo DNMT3A R882 hypo- and hypermethylated motifs as measured by correlation coefficients of the position weight matrices for the respective motifs excluding the CpG dinucleotide. d, Frequencies of DNMT3A R882 hypomethylated motif within 30kb of TSS of the differentially expressed genes between MUT and WT cells in progenitor subsets. P-values were calculated by Wilcoxon rank sum test. e, Frequencies of DNMT3A R882 hypomethylated motif within $10 \mathrm{~kb}, 30 \mathrm{~kb}$ or $50 \mathrm{~kb}$ of TSS of the differentially expressed genes between MUT and WT cells in HSPCs and EPs. P-values were calculated by Wilcoxon rank sum test. f, Ratio of frequencies of DNMT3A R882 hypomethylated motif to those of the control shuffled motif with CpG (Fig. 5e) within $10 \mathrm{~kb}$ of TSS of the differentially expressed genes between MUT and WT cells in HSPCs and EPs. P-values were calculated by Wilcoxon rank sum test. 
bioRxiv preprint doi: https://doi.org/10.1101/2022.01.14.476225; this version posted January 16,2022 . The copyright holder for this preprint (which was not certified by peer review) is the author/funder, who has granted bioRxiv a license to display the preprint in perpetuity. It is made available under aCC-BY-NC-ND 4.0 International license.

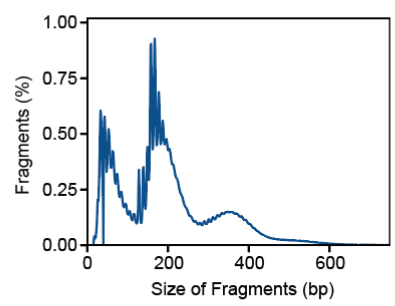

d

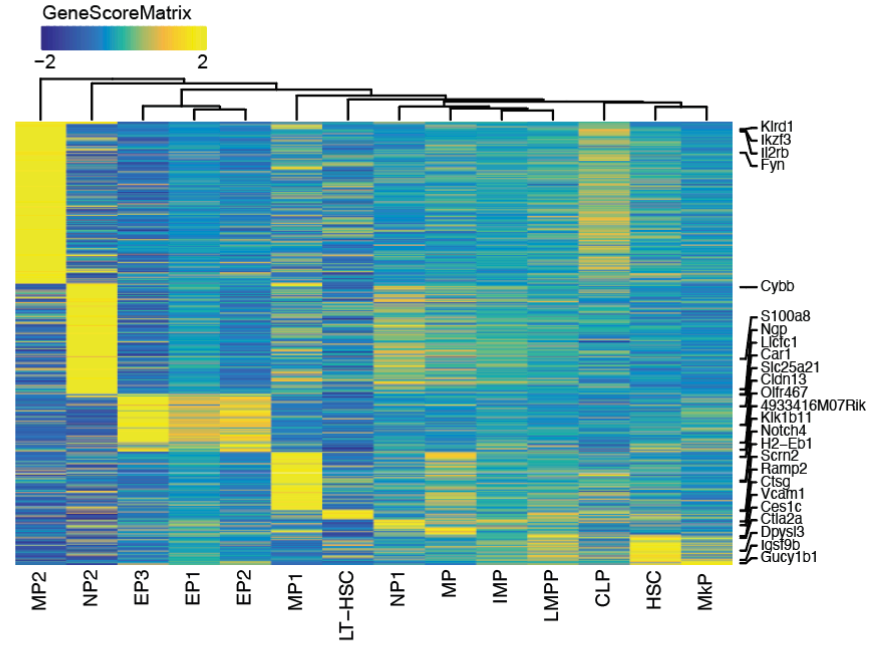

b

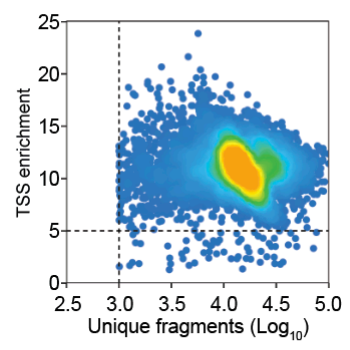

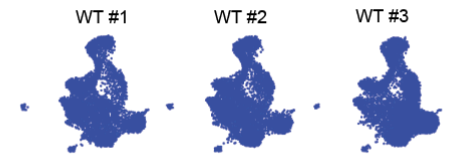

DNMT3A R878H \#1 DNMT3A R878H \#2 DNMT3A R878H \#3

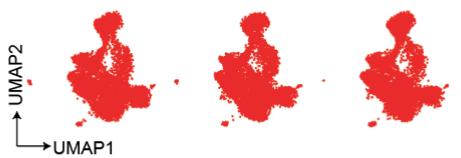

e

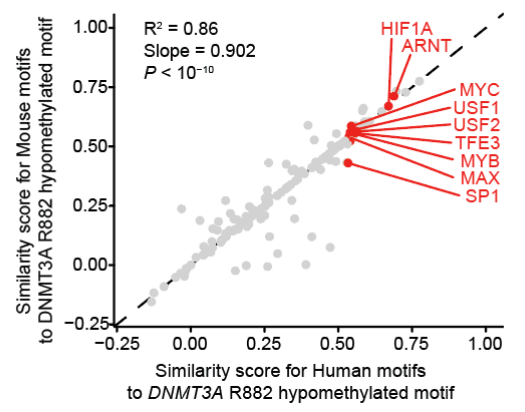

f

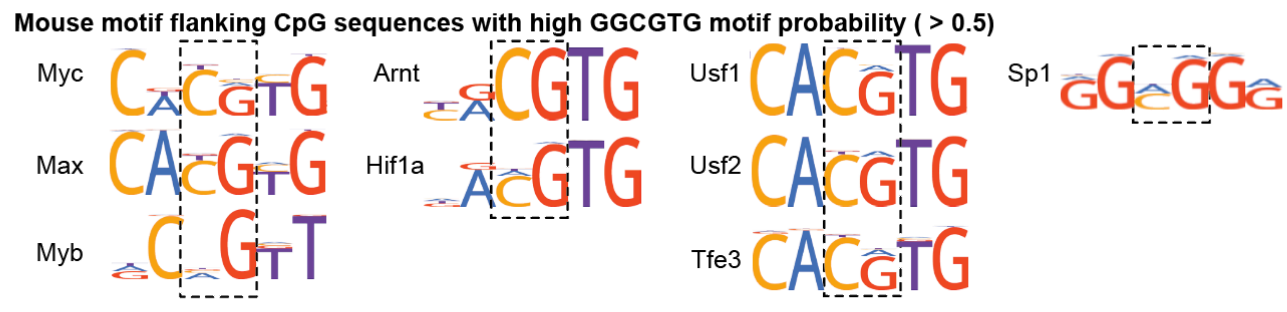

Human motif flanking sequences with high GGCGTG motif probability ( $>0.5$ )

MYC

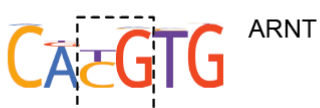

MAX

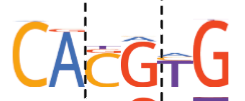

MYB

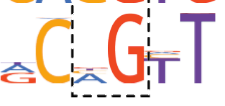

g
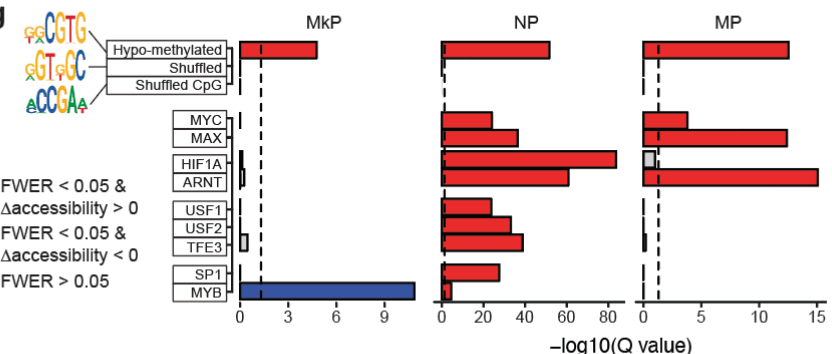

USF1 CA:G

usf2 CAMG

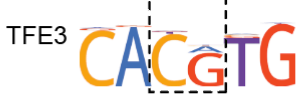

sp1

h

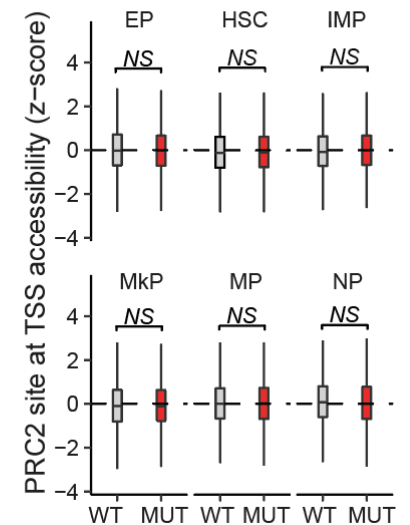

Extended Data Figure 10. Single nucleus ATAC-seq of Dnmt3a R878H Lin-, c-Kit+ progenitors reveals enhanced accessibility of R882 hypomethylated motif and TF motifs with high similarity scores to the hypomethylated motif. a, Distribution of fragment size in snATAC-seq data of Dnmt3a R878H and wildtype Lin-, c-Kit+ progenitors ( $\mathrm{n}=3 \mathrm{in}$ each cohort). b, TSS enrichment of accessible fragments as a function of unique fragments per cell. c, UMAP of integrated datasets Dnmt3a R878H and wildtype Lin-, c-Kit+ progenitors, displayed per sample ( $\mathrm{n}=3$ in each cohort). $\mathbf{d}$, Heatmap of gene accessibility scores for differentially accessible progenitor identity marker genes across progenitor subsets. e, Scatterplot of similarity scores of 
mouse TF motifs versus human TF motifs to the R882-hypomethylated motif (Pearson's correlation, P-value derived from Ftest). f, Binding motifs of mouse and human TFs with high similarity score to the R882-hypomethylated motif and expression in HSPCs (Fig. 5b, HOCOMOCO v11). g, FWER-adjusted P-values for accessibility changes between wildtype and Dnmt3a R878H cells by progenitor identities for hypo-methylated motif and shuffled motifs controls (with and without CpG), as well as motif accessibility deviation of the TFs identified Fig. $\mathbf{5 b}$ (related to Fig. 5f). h, Accessibility of PRC2 targets between wildtype and Dnmt3a R878H and wildtype Lin-, c-Kit+ progenitor subsets. 

available under aCC-BY-NC-ND 4.0 International license.
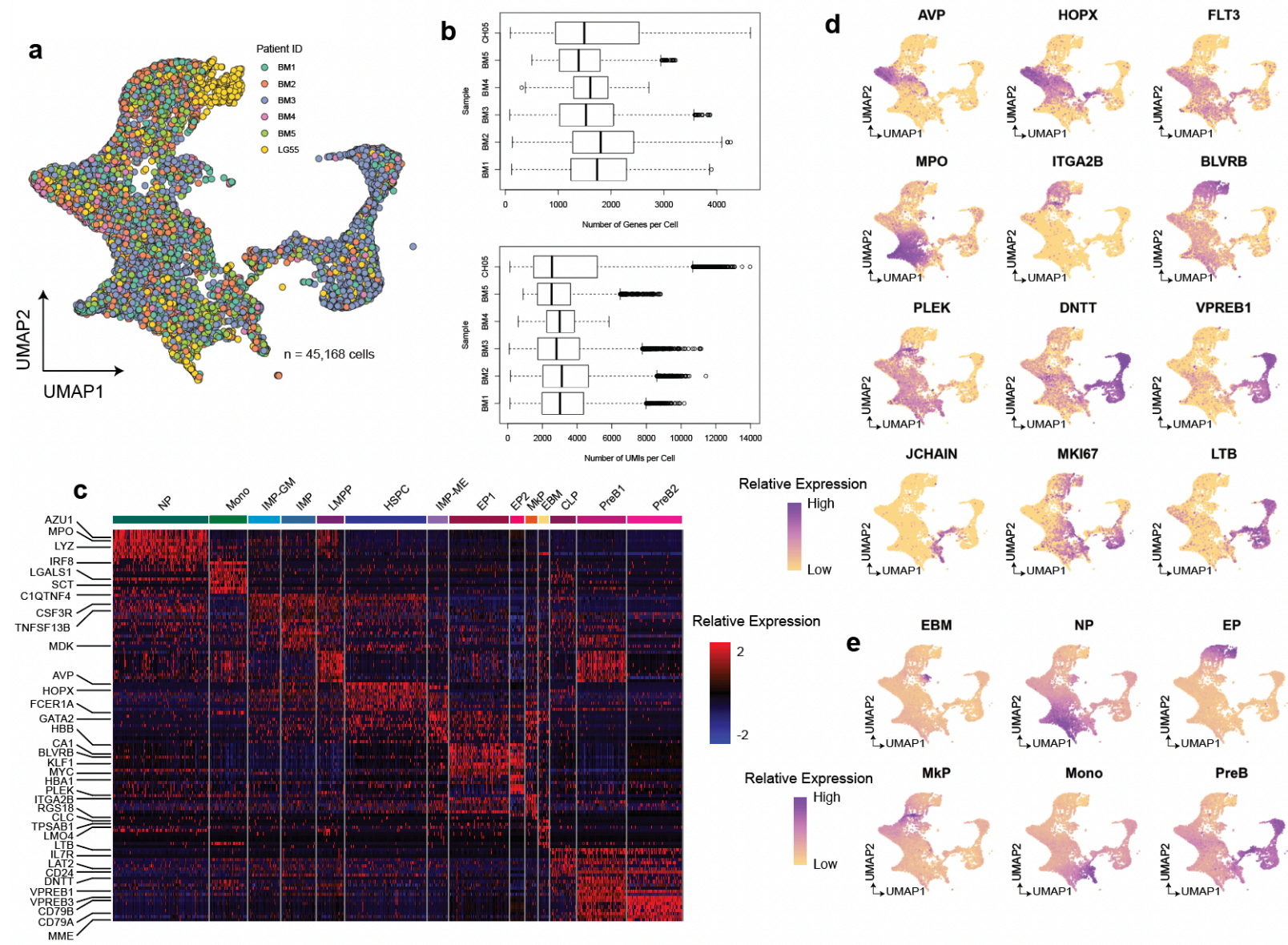

Extended Data Figure 11. Integration of $\mathrm{CHO5}$ and control bone marrow CD34 ${ }^{+}$scRNA-seq data and assignment of progenitor subsets. a, UMAP of CD34+ progenitor cells from samples $\mathrm{CH} 05$ and samples BM01-05 after integration using the Seurat package (online methods). b, Number of genes per cell (top) and number of UMIs per cell (bottom) from CD34 ${ }^{+}$ hematopoietic progenitors by patient sample after QC filters and down-sampling to equivalent geometric means of UMIs per patient. c, Heatmap of top 10 differentially expressed genes for progenitor subsets. d, UMAP representation of CD34 $4^{+}$cells showing cell marker gene expressions. e, Modules from Velten et al. (Supplementary Table 2) are scored and projected onto the UMAP representation of CD34+ cells. 
bioRxiv preprint doi: https://doi.org/10.1101/2022.01.14.476225; this version posted January 16,2022 . The copyright holder for this preprint (which was not certified by peer review) is the author/funder, who has granted bioRxiv a license to display the preprint in perpetuity. It is made available under aCC-BY-NC-ND 4.0 International license.

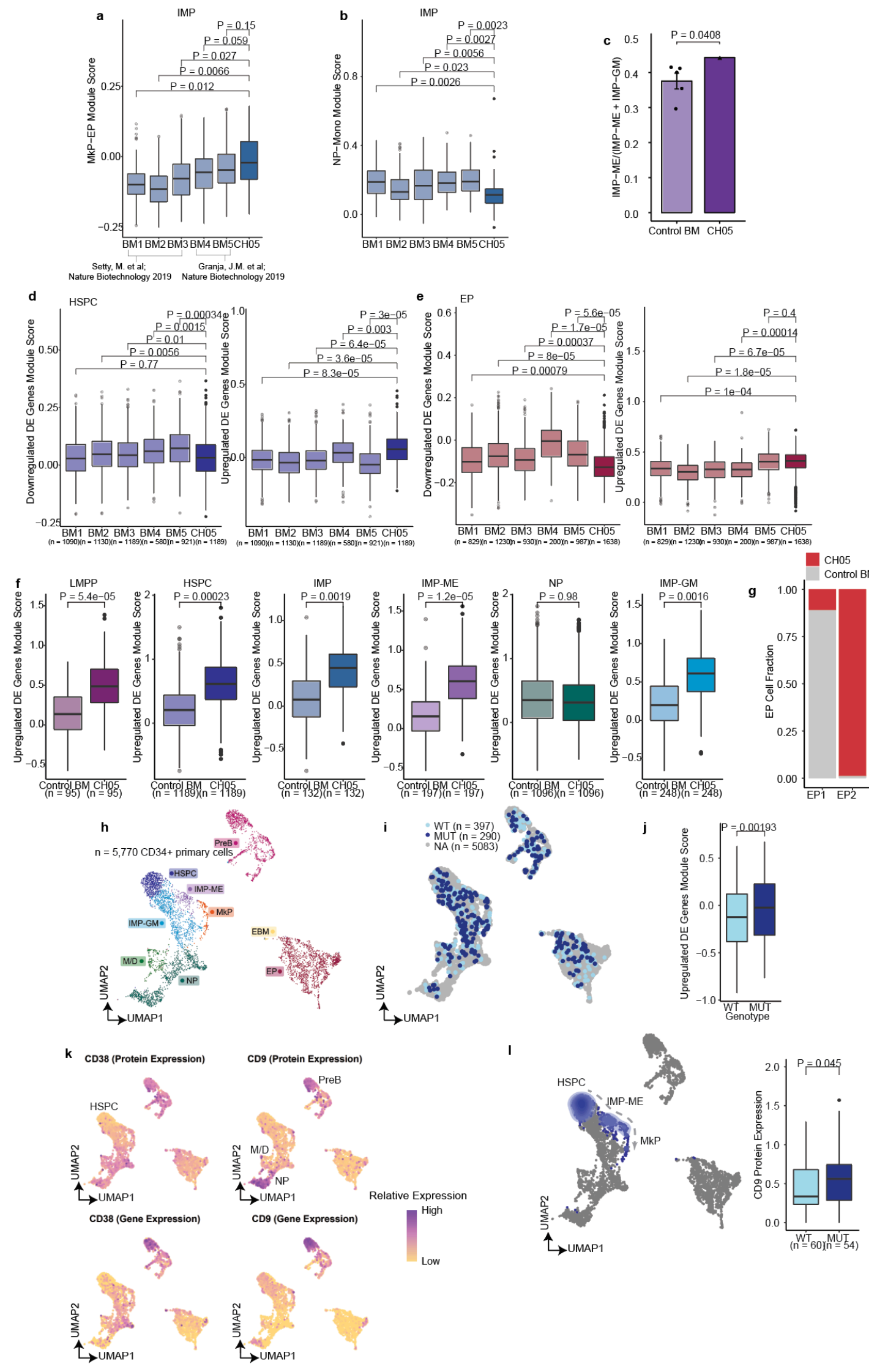

Extended Data Figure 12. Bone marrow clonal hematopoiesis patient sample confirms results from CH01-CH04. a, Perpatient comparison of megakaryocytic-erythroid module scores in control bone marrow versus CH05 IMPs (Supplementary Table 2). Cell number downsampled to the same number $(n=132$ cells per sample). P-values were calculated from likelihood ratio test of LMM with/without CH status. b, Per-patient comparison of granulocytic-monocytic module scores in control versus 
CH IMPs (Supplementary Table 2). P-values were calculated from likelihood ratio test of LMM with/without $\mathrm{CH}$ status. c, Fraction of IMP-ME cells out of all biased IMP (IMP-ME + IMP-GM) cells in control versus CH populations. P-value was calculated from one-sample t-test. d, Per-patient comparison of module scores for differentially down- or up-regulated genes in mutant DNMT3A HSPCs (identified in GoT data, Fig. 3a,c) in control versus CH HSPCs. P-values were calculated from likelihood ratio test of LMM with/without $\mathrm{CH}$ status. e, Per-patient comparison of module scores for differentially down- or up-regulated genes in mutant DNMT3A EPs (identified in GoT data, Fig. 3a,c) in control versus CH EPs. P-values were calculated from likelihood ratio test of LMM with/without $\mathrm{CH}$ status. f, Module scores for genes upregulated in at least 2 cell types (identified in GoT data, Fig. 3b) in control versus $\mathrm{CH}$ cells of major cell types. P-values from likelihood ratio test of LMM with/without $\mathrm{CH}$ status. $\mathbf{g}$, Fraction of control BM or CH05 cells in EP1 versus EP2 cell clusters. h, UMAP of CH05 cells (clustered independently of the control BM samples) with progenitor cell assignments. i, UMAP of CH05 cells with genotyping data for WT ( $\mathrm{n}=397$ cells) and DNMT3A R882 mutant (MUT; $\mathrm{n}=290$ cells). j, Normalized expression of differentially upregulated genes in at least 2 cell types, highlighted in Fig. 3b in wildtype versus mutated cells in CH05. k, UMAP of CH05 cells with protein expression (CITE-seq) and gene expression for CD38 and CD9. l, UMAP of CH05 cells highlighting HSPCs, IMP-ME, and MkPs (left) included in the comparison of CD9 expression in wildtype versus mutated cells (right). 
bioRxiv preprint doi: https://doi.org/10.1101/2022.01.14.476225; this version posted January 16,2022 . The copyright holder for this preprint (which was not certified by peer review) is the author/funder, who has granted bioRxiv a license to display the preprint in perpetuity. It is made available under aCC-BY-NC-ND 4.0 International license.

a

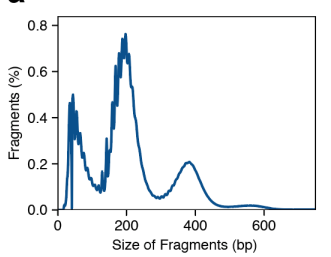

b

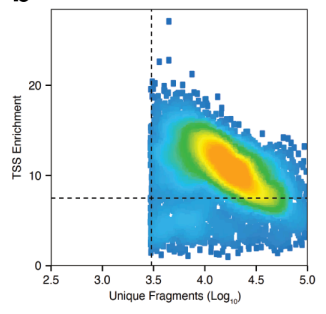

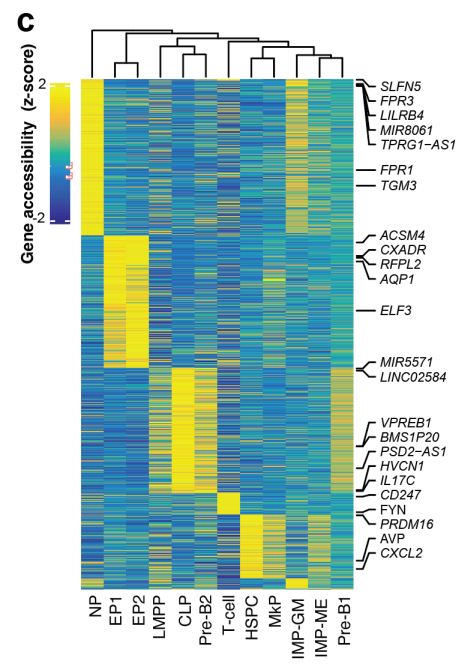

d

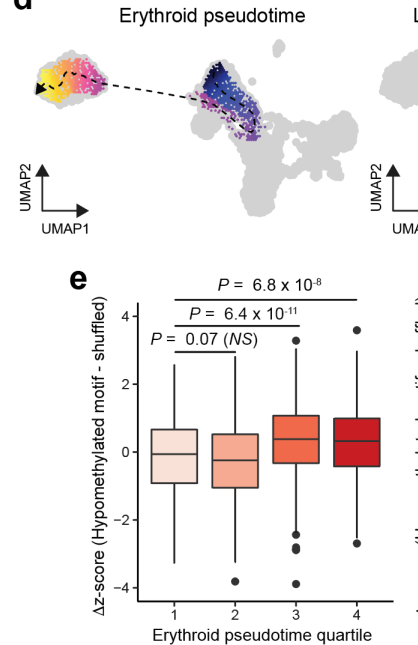

Extended Data Figure 13. Single nucleus ATAC-seq data from bone marrow clonal hematopoiesis reveals enhanced accessibility of hypomethylated motif in mutated erythroid progenitors. a, Distribution of fragment size in snATAC-seq data of patient CH05 with DNMT3A R882 CH. b. TSS enrichment of accessible fragments as a function of unique fragments per cell. c, Heatmap of the gene accessibility scores for cluster marker genes (FDR $<0.01$ and $\log _{2} \mathrm{FC}>1$ ) by cell cluster. d, Pseudotime trajectories for either erythroid (left, $n=1,843$ cells) or lymphoid (right, $n=1,740$ cells) differentiation. e, Difference between hypomethylated and shuffled motif accessibility z-scores across either erythroid $(\mathrm{n}=1,843$ cells) or lymphoid ( $\mathrm{n}=$ 1,740 cells) pseudotime trajectory quartiles. P-values were calculated by Wilcoxon rank sum test. HSPC, Hematopoietic stem and progenitor cell; IMP-ME, immature myeloid progenitor with megakaryocytic/erythroid bias; IMP-GM, immature myeloid progenitor with granulocyte/monocyte bias; LMPP, Lymphoid-myeloid pluripotent progenitor; MkP, Megakaryocyte progenitor; NP, Neutrophil progenitor; CLP, Common lymphoid progenitor; Pre-B1/2, Pre-B cell; EP1/2, Erythroid progenitor. 